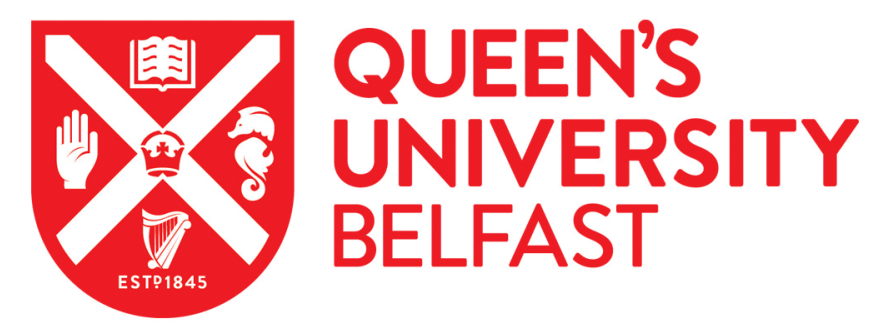

\title{
Get a grip: Individual variations in grip strength are a marker of brain health
}

Carson, R. (2018). Get a grip: Individual variations in grip strength are a marker of brain health. Neurobiology of Aging. https://doi.org/10.1016/j.neurobiolaging.2018.07.023

\section{Published in:}

Neurobiology of Aging

\section{Document Version:}

Peer reviewed version

\section{Queen's University Belfast - Research Portal:}

Link to publication record in Queen's University Belfast Research Portal

\section{Publisher rights}

Copyright 2018 Elsevier. This manuscript is distributed under a Creative Commons Attribution-NonCommercial-NoDerivs License

(https://creativecommons.org/licenses/by-nc-nd/4.0/), which permits distribution and reproduction for non-commercial purposes, provided the author and source are cited.

\section{General rights}

Copyright for the publications made accessible via the Queen's University Belfast Research Portal is retained by the author(s) and / or other copyright owners and it is a condition of accessing these publications that users recognise and abide by the legal requirements associated with these rights.

Take down policy

The Research Portal is Queen's institutional repository that provides access to Queen's research output. Every effort has been made to ensure that content in the Research Portal does not infringe any person's rights, or applicable UK laws. If you discover content in the Research Portal that you believe breaches copyright or violates any law, please contact openaccess@qub.ac.uk. 
Get a grip: Individual variations in grip strength are a marker of brain health

\begin{abstract}
Richard G. Carson ${ }^{\mathrm{a}, \mathrm{b}, \mathrm{c}}$
${ }^{\mathrm{a}}$ Trinity College Institute of Neuroscience and School of Psychology, Trinity College Dublin, Dublin 2, Ireland

${ }^{\mathrm{b}}$ School of Psychology, Queen's University Belfast, Belfast, Northern Ireland, UK ${ }^{\mathrm{c}}$ School of Human Movement and Nutrition Sciences, The University of Queensland, Australia
\end{abstract}

Correspondence: Richard G. Carson

Trinity College Institute of Neuroscience and School of Psychology

Trinity College Dublin

Dublin 2,

Ireland,

E-mail: richard.carson@tcd.ie 


\begin{abstract}
[170 words]
Demonstrations that grip strength has predictive power in relation to a range of health conditions - even when these are assessed decades later, has motivated claims that handgrip dynamometry has the potential to serve as a "vital sign" for middle-aged and older adults. Central to this belief has been the assumption that grip strength is a simple measure of physical performance that provides a marker of muscle status in general, and sarcopaenia in particular. It is now evident that while differences in grip strength between individuals are influenced by musculo-skeletal factors, "lifespan" changes in grip strength within individuals are exquisitely sensitive to integrity of neural systems that mediate the control of coordinated movement. The close and pervasive relationships between age-related declines in maximum grip strength and expressions of cognitive dysfunction can therefore be understood in terms of the convergent functional and structural mediation of cognitive and motor processes by the human brain. In the context of ageing, maximum grip strength is a discriminating measure of neurological function and brain health.
\end{abstract}

Keywords: motor control; frailty; coordination; ageing; longevity; muscle 
The human hand is so beautifully formed; it has so fine a sensibility, that sensibility governs its motions so correctly, every effort of the will is answered so instantly, as if the hand itself were the seat of the will; its action are so powerful, so free, and yet so delicate, as if it possessed quality of instinct in itself, that there is no thought of its complexity as an instrument, or of the relations which make it subservient to the mind

- Bell C. The hand-its mechanism and vital endowments as evincing design. Philadelphia, PA: Carey, Lea and Blanchard; 1833 (pp. 9-10).

\section{Introduction}

While it is widely recognised that frailty is multidimensional construct, reduced grip strength is considered one of its cardinal characteristics. Indeed, the repeated demonstration that measurements of grip strength have predictive power in relation to health outcomes (e.g. Bohannon, 2008; Chainani et al., 2016; Cooper et al., 2011; 2010; Ouden et al., 2011; Rijk et al., 2016; Vermeulen et al., 2011; Y. Wu et al., 2017), even when these are assessed decades later (Ortega et al., 2012; Rantanen et al., 1999), has motivated the supposition that hand-grip dynamometry has the potential to serve as a "vital sign" for middle-aged and older adults (Bohannon, 2008). Central to this belief has been the assumption that grip strength is a simple measure of physical performance (Atkinson et al., 2010; Hamer and Stamatakis, 2013) that provides a marker of sarcopaenia (Sayer, 2006) and an index of musculoskeletal function (e.g. A. J. Campbell and Buchner, 1997) or muscle fitness (W.-J. Lee et al., 2016).

In order for strength to confer functional benefit, a principal requirement is that muscle force is generated with sufficient speed and precision, for example in maintaining an upright posture following a stumble. That which is critical in this regard is the capacity of the central nervous system (CNS) to recruit motoneurons. Of at least equivalent importance in relation to function is the facility to engage multiple muscles in a coordinated goal-directed fashion. It can be readily appreciated therefore that a measure of strength may be extremely sensitive to variations in CNS function - in relation to the recruitment of motor units and the organisation of muscle synergies, and comparatively insensitive to variations in the state of the muscle fibres. In the present paper it will be argued that the assessment of maximum grip strength via hand dynamometry provides a case in point.

Grip strength tends to decline with age. Given the association of the rate at which this decrease occurs with other indices of physical status, grip strength has come to be regarded as an important objective marker of frailty (e.g. Syddall et al., 2003; Xue et al., 2011). There is an emerging recognition that loss of muscle mass is a less prominent contributor to declines in strength than was once believed to be the case (Delmonico et al., 2009; Manini \& Clark, 2012). It is furthermore evident that the proportion of the decline in grip strength that can be attributed to loss of tissue mass in the extrinsic and intrinsic hand muscles, is even lower than for other skeletal muscle groups (Carmeli et al., 2003). That which is of particular importance in determining the expression of frailty is the factors that contribute to a differential loss of strength (Figure 1) beyond that which would be expected on the basis of chronological age (Kallman et al., 1990; Rantanen et 
al., 1998; cf. Sayer et al., 2008). In this consideration, that which appears critical in terms of good health is the relationship that exists between changes in grip strength and other aspects of brain function (e.g. (Christensen et al., 2000). While initial levels of grip strength are greatly influenced by peripheral musculo-skeletal factors (e.g. muscle mass, fibre type) and individual morphology, the central hypothesis advanced herein is that subsequent changes in grip strength across the lifespan are governed to a significant degree by neural mechanisms.

\section{Insert Figure 1 About Here}

The argument to be made is that maximum grip strength testing provides a rather equivocal indication of musculoskeletal integrity, whereas it represents a sensitive measure of neural function. Why is this analysis of more general significance? The practical steps that are taken in an attempt to assess and ameliorate the impact of frailty and cognitive decline in an ageing population depend in part upon the nature of the tools employed to characterise these conditions, but also upon recognition of that which is revealed by these tools. If it is the case that tests of maximum grip strength reflect primarily the functional integrity of the brain, rather than the musculoskeletal system, there are profound implications for the development of therapeutic interventions to combat frailty and cognitive decline.

\subsection{Scope and structure of the review}

The review commences with an introduction to the clinical construct of frailty, and a critique of the manner in which diminished grip strength is conventionally interpreted in this context. This leads to consideration of the factors (neurologic and non-neurologic) that contribute to age- and disease-related declines in grip strength, and to the recognition that these factors do not coincide with the determinants of variations in grip strength among young healthy individuals. A detailed analysis of the motor apparatus of the human hand is then developed - in order to make the key point that effective application of grip force demands highly sophisticated neural control. There follow descriptions of the manner in which older people apply grip force, and of the relationships that exist between variations in grip strength and other indices of age-related changes in muscle coordination. It is surmised that systematic and progressive age-related modifications in neuromuscular control are symptomatic of more general alterations in brain function. Empirical evidence in support of this conjecture is presented, with a particular emphasis on associations between changes in grip strength and cognitive function. It is argued that the tripartite relationship linking grip strength, frailty and cognitive decline, can only be understood by recognising that certain changes in central neurological functions are the common mediating factor. This argument is further supported by a review of studies showing that task-related activity in brain regions playing an obligatory role in the production of coordinated motor output is a pervasive feature of all assessments of 
cognitive function. It is concluded that as neurodegenerative processes affect coherent systems constituted by the brain's intrinsic functional architecture, any erosion of brain health that is expressed phenotypically as diminished grip strength, will have a corresponding causal influence upon manifestations of frailty and impairments of cognition.

\section{Frailty}

As a profusion of classic literary references makes plain, manifestations of frailty have long been readily discerned. Nonetheless, the clinical classification of frailty has a relatively recent history. While the phenomenon is age-related, to be useful as a clinical construct the expression of frailty must necessarily be distinguished from the changes in functional capacity associated with healthy ageing. There have thus far been two principal approaches to this challenge. While these share the common goal of providing a scientific definition of the condition, they differ with respect to the manner in which frailty is conceptualised and assessed.

Fried and colleagues (Fried et al., 2001) proposed that there exists a frailty phenotype with manifestations and biological antecedents that define a distinct clinical syndrome. An incidence of frailty is defined by meeting three out of five phenotypic criteria comprising: unintentional weight loss, self-reported exhaustion, weakness (grip strength), slow walking speed, and low physical activity. Thus conceived, frailty may in principle be differentiated from comorbidity (i.e. multiple chronic conditions) and from disability (Fried et al., 2004).

Alternatively, frailty has been viewed as a non-specific state of dysregulation in multiple physiological systems (Romero-Ortuno and Kenny, 2012), whereby it is the accumulation of deficits (including multiple chronic conditions and disability) in the course of ageing that gives rise to expression of the condition (Mitnitski et al., 2001; Rockwood and Mitnitski, 2007; Rockwood et al., 2006). In this conception, incidence is appraised via a frailty index (FI) representing quantitatively the clinically designated symptoms, signs, diseases, and disabilities that have accreted.

In both schemes frailty is viewed as comprising multiple dimensions and it is assessed accordingly. It is notable therefore that, with respect to the phenotypic definition, reduced grip strength is considered one of the cardinal characteristics (Fried et al., 2001). Indeed there is concordance $($ Kappa $=0.48)$ between the classification of frailty on the basis of the complete set of Fried criteria and handgrip strength alone (van Iersel and Rikkert, 2006). Although the frailty index can be agnostic with regard to the specific deficits that are examined (and may in practice vary substantially in relation to the number of deficits that are assessed), a measure of grip strength is routinely included (e.g. Hubbard et al., 2009; Searle et al., 2008). In light of the statistical convergence of the phenotype and cumulative deficit models (Clegg et al., 2013), it is perhaps unsurprising therefore that grip strength is also a reliable predictor of the frailty index, even when such factors as age, height, body mass index (BMI) comorbidities are controlled (Theou et al., 2011). Similarly, there is a high degree of correlation between 
grip strength and comparable sets of ageing markers, other than those that contribute to the FI (e.g. Syddall et al., 2003). The measure also has the capacity to discriminate early and later stages of frailty (Theou et al., 2011).

Although there is empirical evidence to suggest that diminished gait speed has greater predictive power in relation to classification of the condition (Purser et al., 2006; Rothman et al., 2008; van Kan et al., 2008), it nonetheless remains the case that reduced grip strength is the most common first manifestation of frailty. Whereas grip strength exhibits predictive utility in relation to incipient frailty, this is not a characteristic of the other phenotypic criteria - including speed of walking (Xue et al., 2008). It is furthermore apparent that the rate of decline in grip strength, as opposed to the absolute level of force that is recorded at any specific point in time, is particularly significant in this regard (Xue et al., 2011; 2015).

\section{A critique of the standard interpretation of grip strength in relation to frailty.}

In clinical classifications of frailty, it is frequently the case that grip strength is presented as a "simple" measure of performance (e.g. Atkinson et al., 2010; Hamer and Stamatakis, 2013) amenable to interpretation in terms of musculoskeletal function in general (e.g. A. J. Campbell and Buchner, 1997), and of sarcopaenia in particular (Sayer, 2006). Notwithstanding current debates concerning the definition of sarcopaenia, as will be detailed in the sections that follow, interpretations of grip strength purely in relation to the capacity of muscle fibres to generate tension are problematic. Of at least equal concern is the ambiguity that is inherent in the more widespread contention that handheld dynamometry measures only one component of musculoskeletal performance (İncel, 2014) and is a gauge of "muscle strength" (e.g. Abizanda et al., 2012; Roberts et al., $2011 ; 2014)$. The term muscle strength is employed in some instances to denote explicitly the combined effects of muscle mass and muscle quality ("strength per unit muscle mass"), without any regard to neural factors (e.g. Goodpaster et al., 2006). In others there is clear recognition that strength is a property of the motor system (Enoka, 1988) rather than solely a property of muscle. It is however most frequently the case that the scope of the term is left undefined. To the extent that empirical findings in relation to variations in grip strength are narrated in this way, misattribution in regard to mechanism, and misdirection with respect to the implications for policy and practice are inevitable.

\section{Insert Figure 2 About Here}

As a first step in appreciating the basis upon which changes in grip strength constitute an objective marker of frailty, it is instructive to consider circumstances in which the predictive power of the measure is not readily attributable to variations in muscle structure and function. It is now well established that grip strength as assessed during middle age (Rantanen et al., 2000), later life (De Buyser et al., 2013; Laukkanen et al., 1995; Nofuji et al., 2016; Rolland et al., 2006; Snih et al., 2002; Steiber, 2016; Strand 
et al., 2016), and in the oldest old (Ailshire and Crimmins, 2013; Cesari et al., 2008; Ling et al., 2010) is strongly associated with long-term survival (following adjustment for such factors as age and gait speed). It is conspicuous that the relationship between grip strength and mortality (Figure 2 ) is present in young (mean/median age $<60$ years) cohorts (Leong et al., 2015; see Cooper et al., 2010) for a review), and remains robust at long term follow up (e.g. Sasaki et al., 2007). With regard to such associations, it has been shown repeatedly that the changes in grip strength are not attributable to declines in skeletal muscle mass (Legrand et al., 2014; Metter et al., 2002; Newman et al., 2006) or arm cross-sectional area (Gale et al., 2006), or indeed to other measures of body composition such as body mass index (Ling et al., 2010).

Insert Figure 3 About Here

It is furthermore apparent that measures derived from grip dynamometry are closely correlated with various indices of disability and health related quality of life (Giampaoli et al., 1999; Rantanen et al., 1999; Sayer, 2006; Taekema et al., 2010). While it has frequently been the case that these relationships (Figure 3) have been attributed to the effects of sarcopaenia, there is now a substantial body of empirical work that indicates that they are independent of this factor. For example, Hairi et al. (Hairi et al., 2010) noted that the magnitude of the association between grip strength and physical activity in activities of daily living was markedly greater than the associations with lean muscle mass (assessed by dual X-ray absorptiometry (DXA)) or muscle quality, and concluded that strength alone is the best clinical measure with which to assess functional limitations and physical disability. The utility of grip strength as a potent predictor of future mobility impairment is also largely independent of lean muscle mass (McLean et al., 2014). Similarly, Cawthon et al. (Cawthon et al., 2009) in studying a sample of more than three thousand men and women aged between 70 and 80 years determined that individuals with the weakest grip strength exhibited the greatest risk of hospitalization. In contrast, lean muscle mass (assessed using DXA) and computed tomography (CT) derived estimates of muscle cross-sectional area and muscle density were not associated with risk of hospitalization (see also Legrand et al., 2014). In the context of the BELFRAIL study that engages individuals aged 80 years and older living in Belgium, Legrand et al. (Legrand et al., 2013) determined that performance on a standard physical performance battery was associated with grip strength but not with skeletal muscle mass. These results are all consistent with the more general finding that in both sexes, declines in grip strength with age are largely accounted for by factors other than loss of muscle mass (Baumgartner et al., 1999; Kallman et al., 1990). 
The same conclusion may be drawn on the basis of intervention studies in which the principal aim has been to increase muscle mass, with the ostensible purpose of increasing functional capacity. In the context of a large scale, double-blinded, placebocontrolled study, the administration of testosterone replacement therapy for 6 months improved lean body mass and reduced fat mass, without a concomitant increase in grip strength (Srinivas-Shankar et al., 2010). This outcome is consistent with that of a larger body of work that has demonstrated that with the exception of some older hypogonadal men (Page et al., 2005), testosterone induced increases in skeletal muscle mass do not enhance grip strength (e.g. Emmelot-Vonk et al., 2008; Nam et al., 2018; Snyder et al., 1999; Tenover, 1992; Travison et al., 2011; Wittert et al., 2003) (Figure 4). The more general point can be made that age-related decreases in muscle mass have a specific etiology, and this provides only a partial explanation of declines in functional capacity (O'Connell and F. C. W. Wu, 2014; Tieland et al., 2018).

There exists a complementary body of literature indicating that increases in grip strength may be accrued in the absence of gains in muscle mass. It has been shown for example that twelve weeks of handgrip training undertaken by individuals diagnosed with rheumatoid arthritis (mean age 50 years), gives rise to profound increases in maximum grip strength in the absence of accompanying changes in (MRI derived) measures of muscle mass (Speed and R. Campbell, 2012). In a similar vein, there is a large corpus of work dating back to the nineteenth century (Scripture et al., 1894) that documents increases in grip strength brought about solely through training undertaken by the opposite limb (N. G. Boyes et al., 2017; Shields et al., 1999; Stromberg, 1986; Yasuda and Miyamura, 1983) (Figure 5). Since in these circumstances there is no accompanying muscle hypertrophy (i.e. for the non-training limb), the dissociation between the induced alterations in grip strength and the state of the muscle fibres is complete, and it must be surmised that the increase in functional capacity is brought about by neural factors alone. Indeed, even in circumstances in which muscle atrophy is induced by an extended period (three weeks) of immobilization, a training programme consisting of maximal voluntary effort handgrip contractions performed by the opposite limb maintains handgrip strength (Farthing et al., 2011).

Insert Figure 5 About Here

On the basis of the foregoing, it is contended that the predictive utility of grip strength in relation to age, frailty, and morbidity is largely independent of muscle integrity. In the sections that follow, the case will be made that the relationships between grip strength and functional capacity are more profitably understood in terms of neural mechanisms. While it is undeniably the case that musculo-skeletal factors (e.g. muscle mass, fibre type) have a significant bearing upon initial levels of grip strength, it does not necessarily follow that variations in grip strength (e.g. with ageing) are (primarily) accountable in these terms. It cannot be assumed that declines in grip strength in disease states are attributable to those factors that account for differences in grip strength between healthy individuals. In the event that there is only a partial representation of the 
factors that contribute to variations in grip strength, there will necessarily be an impoverished understanding of their relationship with frailty and cognitive decline.

\section{Dynapenia and sarcopenia}

In 1989, the term 'sarcopenia' (Greek 'sarx' or flesh + 'penia' or loss) was coined by Irwin Rosenberg to denote decline of muscle mass (Rosenberg, 1989). In spite of this definition having served the scientific community well, faced with overwhelming evidence that the relationships between adverse health outcomes and strength are largely independent of muscle mass (e.g. Abellan van Kan et al., 2009), there have been numerous recent attempts to extend its scope. For example, European Working Group on Sarcopenia in Older People (EWGSOP) (Cruz-Jentoft et al., 2010) offered a definition of "low muscle mass and low muscle function" - the latter expressed in terms of strength (e.g. grip) or performance (e.g. gait speed). The Asian Working Group for Sarcopenia (AWGS) prescription includes cut-off values for measurements of muscle mass, handgrip strength and usual gait speed (Chen et al., 2014). In a similar vein the International Working Group on Sarcopenia (IWGS) definition (Fielding et al., 2011) encompasses "age-associated loss of skeletal muscle mass and function". For diagnostic purposes, muscle function is in this case expressed simply in terms of low gait speed. The Society for Sarcopenia and Cachexia and Wasting disorders has decided that sarcopenia is "reduced muscle mass, with limited mobility". In recognition of the fact that low muscle mass alone is not reliably associated with function and disability, the FNIH Sarcopenia Project criteria are defined in terms of: appendicular lean mass (ALM: sum of lean mass in the arms and legs) divided by body mass index (BMI); gait speed; and grip strength (Studenski et al., 2014).

To the extent that they capture differing groups of people, these inconsistencies in definition necessarily give rise to operational difficulties (Bijlsma et al., 2013), and yield distinct patterns of association with other indices of health status and functional capacity (Dam et al., 2014; O'Connell and F. C. W. Wu, 2014). The more far-reaching concern is that the adoption of a variety of definitions - all of which deviate from the clear circumscription provided by the original, impedes conceptual clarity, particularly with respect to the contributions to functional capacity of processes that differ fundamentally in aetiology and pathophysiology from declines in muscle mass (Hepple, 2012). In the present paper sarcopenia is used in the original strict sense of the term (exceptions are indicated by the use of quotation marks). In doing so, and having highlighted the limited explanatory power of changes in muscle mass in relation to frailty, attention can be directed precisely to the other key factors that are instrumentally related to the manifestation of strength.

In recognition of both the disassociation between age-related loss of muscle mass and strength, and indications that changes in strength and muscle mass resulting from physical training (or disuse) do not follow the same time course, Clark and Manini (B. C. Clark and Manini, 2008; cf. Morley et al., 2011) proposed that the term "dynapenia" (from the Greek for "poverty of strength, power, or force") be used to designate agerelated loss of neuromuscular strength. In opting for the expression "neuromuscular 
strength" rather than "muscle strength", Clark and Manini were seeking to emphasise that a host of neurologic (as opposed to skeletal muscle) factors play a determining role in relation to the production of maximal voluntary force.

A general lack of consensus notwithstanding, maximum grip strength is an element of many contemporary definitions of "sarcopenia". For example, both the AWGS and the EWGSOP definitions embody an algorithm that includes measures of maximum grip strength, gait speed, and absolute muscle mass. The challenges to interpretation and operational difficulties that arise from the inclusion of maximum grip strength in a definition of "sarcopenia", i.e. when it is treated simply as a measure of skeletal muscle function, are readily illustrated through reference to the concept of dynapenia. In the context of the I-Lan Longitudinal Aging Study (ILAS) in which 731 older (> 50 years) people were engaged, the AWGS defined composite measure of "sarcopenia" was found not to be associated with variations in global (MMSE) or individual facets of cognitive function. In contrast, those sub-components of the "sarcopenia" measure subject to the influence of neuromuscular factors - and thus sensitive to dynapenia (gait speed and maximum grip strength), exhibited independent associations with both the global index of cognition (MMSE), and additional individual indices of cognitive function. For grip strength these included the Verbal Fluency Test and the Digits Backward Test (the latter used to assess the domain of attention) (Huang et al., 2016). Individuals with a diagnosis of diabetes-related dementia (DrD), but without a diagnosis of Alzheimer's disease (AD) or vascular dementia, exhibit higher levels of dynapenia than individuals with AD - in the absence of differences in a skeletal muscle mass index or the AWGS defined measure of "sarcopenia" (Hirose et al., 2017). Furthermore, variations in the capacity for independent living in the community (Tokyo Metropolitan Institute of Gerontology Index of Competence (TMIG-IC)) are associated with the assessed level of dynapenia, but not with the estimated degree of "sarcopenia" (derived using the AWGS criteria) (Iwamura and Kanauchi, 2016). Similarly, individuals drawn from the Fragility in Brazilian Elderly Research Network (FIBRA Network) who exhibited only dynapenia - defined on the basis of maximum grip strength (i.e. did not satisfy a criterion of low muscle mass) were characterized by physical disability in basic activities of daily living (BADL) and instrumental activities of daily living (IADL). No such association was present for "sarcopenia" defined using the EWGSOP criteria (Neves et al., 2018; see also Santos et al., 2017; cf. da Silva Alexandre et al., 2014).

Not only do contemporary definitions of sarcopenia lack the conceptual clarity of the original, in respect of their operational deployment, the distinct aetiologies to which their elements are sensitive necessarily undermines aspirations to use a diagnosis of sarcopenia as the basis for prescription or appropriately focussed intervention (Hepple, 2012). In short, the steps that might be taken to address a decline in muscle mass are quite distinct from those indicated by deficits in neuromuscular control. This is an issue to which further consideration will be given in the closing section.

A motor unit is designated as those muscle fibres (which may be dispersed widely throughout a muscle) that are innervated by a single motor neuron. Although all actions are mediated by integrative interactions between elements located at multiple sites within the CNS, motor neurons provide the only available output channel (Liddel and 
Sherrington, 1925). In considering the various neurologic factors that lead to variations in maximum grip strength, it is therefore necessary to assess the facets of neuromuscular activation - the process by which muscular force is generated via recruitment and rate coding of motor units (e.g. D. J. Clark and Fielding, 2012), which may play a contributory role.

Aside from such factors as reduced efficiency of signal transmission at the neuromuscular junction (Balice-Gordon, 1997; Deschenes et al., 2010; Jang and Van Remmen, 2011), and deficiencies in excitation-contraction coupling (Delbono, 2003; Plant and Lynch, 2002) - which have been studied largely in animal models, it has been established that older adults exhibit specific deficits in neuromuscular activation (Manini et al., 2013). These extend to impairments in the recruitment of lower motor neurons in the elicitation of spinal reflexes (e.g. deVries et al., 1985; Scaglioni et al., 2002), and in response to descending drive from higher motor centres in the context of voluntary contractions (B. C. Clark et al., 2015). With respect to the latter, it has been estimated that the age-related deficit in the proportion of the motor neuron pool that can be recruited voluntarily exceeds $10 \%$ in those aged over 65 years (Morse et al., 2004; Stevens et al., 2003), and may be very much greater in the very old ( $>85$ years) (Harridge et al., 1999). Nonetheless, impaired voluntary activation is not an obligatory consequence of aging (D. J. Clark et al., 2010), and the degree to which such deficits are expressed depends on the muscle groups that are examined (Klass et al., 2007; 2005; Russ et al., 2012).

The more general question that arises in the context of the present review is whether deficits in neuromuscular activation (i.e. recruitment and rate coding of motor units) are sufficient to account for the contribution of neurologic factors to age-related declines in maximum grip strength. The alternative perspective is that it is necessary to also consider the specific details of the task context in which recruitment of motor neurons occurs as maximum grip strength is assessed. In this regard, there are multiple observations that individual differences in maximum grip strength exhibit only low levels of association with other measures of strength that place equivalent demands upon the capacity for neuromuscular activation (Felicio et al., 2014; Jenkins et al., 2014; Samuel and Rowe, 2012). 


\section{The marvel of the human hand}

To chip a flint into the rudest tool, or to form a barbed spear or hook from a bone, demands the use of a perfect hand. Descent of Man, Charles Darwin (1871: 138) (Darwin, 1871)

It has long been recognised that the capacity of the human hand for prehensile movement represents one of the most highly evolved functions in the animal kingdom (Ohtsuki, 1981), page 21). There are 19 bones, 17 articulations, and 19 muscles situated entirely within the hand - these permit the independent action of each phalanx, and a further 20 tendons activated by "extrinsic" muscles in the forearm (Figure 6). The latter may be divided into 5 muscles that give rise to pronation-supination, 6 that generate movements of the wrist, and 9 that produce flexion or extension of the digits (Tubiana, 1981a,, Chapter 4).

Insert Figure 6 About Here

While the morphology of the hand provides for great adaptability in terms of the variety of movements that can be undertaken, and a level of redundancy sufficient to permit tasks to be accomplished in many different ways, there is an associated cost in terms of the large number of elements (degrees of freedom) that must be controlled by the CNS. Stated concisely, the motor apparatus of the human hand is vastly more complex than is necessary for any of the specific tasks that it will be called upon to perform (Santello et al., 2013). The means by which the many available degrees of freedom are harnessed in giving effect to the myriad functions afforded by a system such as the hand has been recognized as a central problem of motor control. Necessarily its solution requires coordination - "the organisation of control of the motor apparatus" (Bernstein, 1967), p. 355), through the formation of "structural units" - assemblies of elements united in relation to a common goal (Gelfand and Tsetlin, 1966). Normally multiple motor units (and thus muscle fibres) are engaged together. Muscle coordination is therefore the organisation of control of motor units (Carson, 2006), and it is through this means that the extraordinary power, subtlety, and flexibility of the human hand is realised.

Muscle coordination is achieved via synergies - defined operationally as mechanisms employed by the CNS to coordinate groups of motor units into functional assemblies (e.g. Windhorst et al., 1991). The challenge of synergy formation, and in particular the requirement for constant and mutual adjustment of synergy composition in the context of dynamic actions performed in an ever changing environment, is further accentuated by the presence of (biomechanical) constraints imposed by musculo-skeletal anatomy. For example, it is impossible to move a single articulation of the hand or generate torque at a single joint without generating ("superfluous") torques acting at other joints. Such constraints dictate that any action performed by the hand requires the 
recruitment of a host of motor units with diverse mechanical actions (Weiss and Flanders, 2004). Necessarily therefore, all behaviours executed by the hand, even those that may appear on initial inspection to be "simple" - such as gripping an object with maximal force, require the coordinated engagement of large numbers of motor units, including many which if considered in isolation may seem to be unrelated to the goal of the task. The quality of this coordination is however the critical determinant of the level of performance that can be achieved.

\subsection{Proximal constraints on maximum grip strength}

In view of the widespread assumption that the voluntary generation of maximum grip force may be considered a simple motor task, it is worth considering in some detail the impact of the biomechanical context in which the action is performed, and the consequential demands that are placed upon the neuromuscular system. As an example, for any object (such as a grip dynamometer) to be held immobile, the net force generated by muscle activation (and by passive tissues) must counteract precisely the force of gravity. In most cases maximum grip strength testing is conducted with the elbow flexed at $90^{\circ}$ - the angle at which the (extension) moment of force acting at the elbow due to the weight of the dynamometer is maximum (Figure 7A). Counteracting elbow flexion torques must therefore be generated through the activation of muscles that cross this joint. The angle of the joint further dictates the level of descending drive required to generate a given level of force by virtue of the length-tension relationships of the muscles that are engaged (Gordon et al., 1966). It may also alter the relative contribution of afferent activity to motoneuron excitability (Nielsen and Sinkjaer, 2002). Consistent with these considerations, maximum grip strength values are sensitive to the position of the (unsupported) elbow joint - being larger when it is fully extended than when it is maintained at an angle of $90^{\circ}$ (Ridan et al., 2003). This is the case even though there are no muscles that cross both the elbow and the digits. It is therefore notable that the degree of forearm support provided during maximum grip strength testing may vary markedly between protocols.

Insert Figure 7 About Here

In recruiting motor units and in shaping the timing of their activation for the purpose of generating manual forces, the CNS is thus bound by the totality of constraints arising from the (structural and functional) organisation of the motor apparatus. As a consequence, the level of performance that is ultimately achieved depends fundamentally upon the manner in which the CNS can satisfy the demands imposed by these interdependencies upon the planning and instantiation of muscle synergies. In the context of maximum grip strength testing, the implications of this fact (e.g. El-Sais and Mohammad, 2014) assume profound significance. 
In his encyclopedic treatise upon the hand, Tubiana (Tubiana, 1981b) noted that in order for maximum grip force to be generated, the wrist must be maintained stable in slight extension and ulnar deviation. The functional importance of this factor is emphasised by the observation that in full flexion of the wrist, the grip force that can be produced is reduced substantially (Ambike et al., 2013; Burssens et al., 2017; Napier, 1966). A similar outcome is brought about by paralysis of the wrist flexors or extensors. In recognising that active control of the wrist is fundamental to the generation of grip force, Tubiana remarked that the level of functional assimilation of elements distal to the elbow is sufficient to conclude that they constitute "but one physiological unit" (1981, p. 39). Indeed, the integration of the actions of the wrist and forearm with those of the hand is of such importance that the wrist has been referred to as the 'key joint of the hand' (Bunnell and J. H. Boyes, 1970).

The intimacy of the relationship between the control of the wrist joint and the generation of grip force arises from the structural configuration of the hand, wrist, and forearm. Flexion of the digits is brought about through the activation of muscles intrinsic to the hand, and by muscles extrinsic to the hand that have their origins in the forearm. As the tendons of these extrinsic muscles cross the wrist before reaching the digits, their recruitment also generates torques at this joint. The maintenance of a stable posture therefore requires counteracting torques to be produced by the "dedicated wrist movers" (extensor carpi ulnaris (ECU), extensor carpi radialis longus (ECRL), extensor carpi radialis brevis (ECRB), flexor carpi radialis (FCR), and flexor carpi ulnaris (FCU)). For example, the action of ECRB counteracts the wrist flexion moment arms of the flexor digitorum superficialis (FDS) and flexor digitorum profoundus (FDP), whereas ECRL activity counterbalances ulnar deviation (Johanson et al., 1998). The functional consequences arising from the engagement of these muscles can be appreciated from the observations that relative to a neutral position, $45^{\circ}$ of wrist flexion decreases maximum grip force by $40-50 \%$ (Mogk and Keir, 2003), Figure 7B). When individuals are afforded the opportunity to self-select the posture of the wrist, a configuration characterized by $35^{\circ}$ extension and $7^{\circ}$ ulnar deviation (on average) is adopted. All deviations from this posture give rise to a reduction in maximum grip strength (O'Driscoll et al., 1992; see also Z.-M. Li, 2002). The synergistic nature of the control scheme implemented by the CNS in such task contexts is further highlighted by the observation that when holding an object at different wrist joint angles, the activation of the intrinsic hand muscles is modulated in parallel with that of the extrinsic muscles, even though the lengths and moment arms of the former are not altered by variations in wrist posture (Johnston et al., 2010).

Although the position of the wrist joint has a significant bearing on the force that can be produced by the digital flexors, of particular importance in generating maximum grip force is the requirement that joints proximal to the fingers are stabilized effectively. In circumstances in which there is movement of the wrist, the force that can be generated at a given position is approximately $30 \%$ less than that recorded when the joint is static (LaStayo and Hartzel, 1999). In cases in which a grip dynamometer is held the standard position - with the elbow flexed at $90^{\circ}$, additional external moments of gravitational force acting at the wrist due to the weight of the device, must also be counteracted actively in order to maintain joint stability. Such requirements not only dictate the activity profiles of 
the dedicated wrist movers, they also determine the levels of force that can be generated by the digits (Rearick et al., 2003). Necessarily any deficits in the stabilization of the wrist joint in the face of the external load will attenuate the forces that can be applied by the fingers.

In considering the demands placed upon the CNS by the organization of the motor apparatus, it is critical to appreciate that the selection of motor synergies appropriate to the demands of the task at hand must be prospective, rather than simply reactive. The term anticipatory postural adjustment (APA) has been coined for actions that compensate in advance for the disturbances to equilibrium brought about by voluntary movement (Massion, 1992). While APAs have classically been studied in tasks that have the potential to induce significant displacements of the centre of mass, (e.g. Aruin and Latash, 1995; Belen'kiı̌ et al., 1967; Bouisset and Zattara, 1987), it is clear that the formulation of postural synergies that support the generation of maximum grip force also depends critically on CNS mechanisms that implement forward planning. The study of APAs engaged in stepping and other tasks that alter the position of the base of support has seen an understandable emphasis on the potential role of sub-cortical circuits (cf. Goldberger, 1969). The impaired ability to prospectively adjust multi-finger synergies that is exhibited by patients with olivo-ponto-cerebellar atrophy (OPCA) and Parkinson's disease also points to a role of sub-cortical structures in the anticipatory feed-forward control of the hand (Park et al., 2013; 2012). It is nonetheless increasingly clear that in the context of power grips a fronto-parietal cortical network is also engaged during both grasp planning and execution (Alahmadi et al., 2016; King et al., 2014; Westerholz et al., 2013). In particular, the anticipatory control appears to draw on resources that extend beyond the classical motor network, to encompass other regions that play a role in cognition. It is evident that the characteristics of APAs preceding self-initiated arm (Asaka and Y. Wang, 2008; Inglin and Woollacott, 1988; Man'kovskii et al., 1980; Rogers et al., 1992; Stelmach et al., 1990) and hand (Halla B Olafsdottir, 2008; Olafsdottir et al., 2007a) movements exhibited by older people are clearly discriminable from those of young adults, and are accentuated in those with subclinical manifestations of neurological deficits (Woollacott and Manchester, 1993). In circumstances in which a concurrent cognitive task is introduced, alterations in anticipatory postural control in older people are in general more pronounced (Laessoe and Voigt, 2008).

\subsection{Distal constraints on maximum grip strength}

In effecting a power grip, the positions of the fingers and the torques that they transmit must both facilitate the application of force to the object, and accommodate the external forces acting upon it. Although the extrinsic flexor muscle FDP makes the largest contribution to grip force, muscles intrinsic to the hand play a critical role in altering its shape - by rotating, abducting, and adducting the phalanges, in a manner that contributes to the satisfaction of these dual task demands. Foremost among the actions that they perform is alignment of the fingers, such that the work of the extrinsic muscles in applying force upon the object is best exploited. Interossei with major first phalangeal attachments to bone, including the first dorsal interosseus (FDI) muscle, also contribute to grip force through their action as metacarpophalangeal-joint flexors. Since these 
muscles produce moments of force in addition to flexion, other interossei with actions that oppose the direction of rotation, such as the first palmar interosseus, must therefore also be recruited in a coordinated fashion (Backhouse, 1968; Long et al., 1970). Whereas the number of muscles that actuate the elements of the hand is finite, if the relative timing of their recruitment is also considered, the potential number of muscle synergies is very large indeed (Santello et al., 2013). It might be supposed therefore that there exists scope for the CNS to readily generate patterns of recruitment that maximize the functional contribution of agonist muscles. When examined empirically however, it becomes clear that the neural control required to deal with the complexity of the motor apparatus requires significant concessions to the constraints imposed by musculo-skeletal anatomy. It is the efficiency with which these constraints can be accommodated that determines the ultimate levels of performance that can be achieved.

It has long been appreciated that the maximum force that can be produced by each finger decreases in proportion to the number of other fingers that are engaged simultaneously (Kinoshita et al., 1995; Ohtsuki, 1981). When four fingers contribute to the grip, the maximum force that can be generated by each digit is typically less than $50 \%$ of that produced when it is engaged in isolation (Z. M. Li et al., 1998a; 1998b). In these circumstances the level of neural drive received by the actuating muscles is also diminished (Ohtsuki, 1981), suggesting that the limiting factor is neural rather than biomechanical. In short, the CNS does not concurrently activate all finger actuating muscles to their maximum degree (Z. M. Li et al., 1998b; 1998a; Yu et al., 2010). Of especial importance in the present context is the observation that the diminution of the force applied by the individual digits during four-finger tasks is larger in older persons than in the young (S. Li et al., 2003; Shinohara et al., 2003a; 2003b) (Figure 8).

\section{Insert Figure 8 About Here}

In light of the fact that variations in the forces applied by the individual digits in multi-finger maximum grip tasks are both sub-maximal and negatively correlated, Li et al. (Z. M. Li et al., 1998b) hypothesized that the CNS engages in "force sharing" as a means of minimizing the resulting moment acting to rotate the hand, thus obviating the requirement that compensatory torques be generated by muscles acting at the wrist and radioulnar joints. Examined empirically, it has been determined that when four digits apply force simultaneously, the resultant moment is minimal with respect to an axis passing approximately along the line of the middle finger (Z. M. Li et al., 1998a). Since in maximum grip tasks there is typically the requirement to stabilise the gripped object in space, the generation of disproportionate force by one or more fingers compromises the satisfaction of this requirement, gives rise to increased compensatory activity in other muscles and thereby detracts from the total force applied. Similarly, when electrical stimulation of the intrinsic and extrinsic flexor muscles is imposed upon voluntary contraction, maximal grip force diminishes (Boisgontier et al., 2010). Satisfaction of the functional goal - that of applying the maximum total grip force can thus only be realised when the individual digits apply levels of force well below those which can be achieved 
when they are engaged in isolation. The more general point is that when activated in the context of a functional synergy (i.e. in all natural actions) the central neural drive directed to the individual motor units of which the synergy is composed is strictly bound by the demands of muscle coordination. The supposition that it is the degree to which these demands can be satisfied which determines the level of performance achieved in maximum grip strength testing, is further supported by the observation that the extent of the gains derived for the non-training limb are largely independent of the intensity of grip strength training undertaken by the training limb (N. G. Boyes et al., 2017; Shields et al., 1999), Fig 4). The most parsimonious explanation of this finding is that the increases in strength are mediated by enhanced coordination of muscle activation (e.g. Barry and Carson, 2004a).

\section{How do older people apply grip force?}

Over the course of healthy ageing the characteristics of grip force application change in several ways. To a large extent these changes extend beyond simple deficits in muscle force generating capacity, to reflect systematic and progressive alterations in neuromuscular control that are symptomatic of more general alterations in brain function. Upon gripping an object, older adults often misalign the application and directions of the force vectors produced by individual digits (Parikh and Cole, 2012). The resulting moments of force tend to rotate the object. As noted above, both the deviations from optimal joint angles that result from such rotations, and the fluctuations associated with the maintenance of unstable orientations, lead to profound diminution of maximum grip force (LaStayo and Hartzel, 1999). Similarly, when applying force to a dynamometer simultaneously with four fingers, older adults exhibit high levels of variability in the orientation of proximal limb segments, including the forearm in pronation/supination (Shinohara et al., 2004). That these deficits are of neural origin is emphasised by the fact that minimal levels of practice (extending over a period of minutes) can give rise to substantial improvements in such aspects of task performance (Parikh and Cole, 2012).

With respect to the distal effectors (i.e. the phalanges), older adults display deficiencies in both force- and moment-stabilization (Olafsdottir et al., 2007b; Shinohara et al., 2004; 2003a), which reflect a reduced capacity to coordinate the forces applied by the individual digits (Shim et al., 2004). As emphasised previously, the patterns of muscle coordination that serve to effectively regulate forces produced by the hand are necessarily both anticipatory and compensatory. Healthy young adults acting in anticipation of an increase in the coordinated force applied by digits II to V, exhibit reductions in the covariation of signals representing control of the individual fingers 100$150 \mathrm{~ms}$ in advance of elevations in the compound force level (Olafsdottir et al., 2007a; 2005; Zhang et al., 2006). In older adults, such prospective adjustments in the formulation of muscle synergies are diminished in extent, and occur $(50-100 \mathrm{~ms})$ later than in the young (Halla B Olafsdottir, 2008; Olafsdottir et al., 2007a) - indicating a decreased capability for feedforward control.

As a corollary of the observation that the magnitude of the force deficit (i.e. the diminution of the force applied by the individual digits during four-finger tasks) increases 
with age, it has been noted repeatedly that the finger force vectors generated by older adults deviate from the directions required by the task to a greater degree than is the case for younger persons (Kapur et al., 2010; Park et al., 2011). Furthermore, it is a general finding that with ageing, static prehension is increasingly characterized by the energetically wasteful generation of high internal forces that increase the stiffness of the hand (Diermayr et al., 2011; Solnik et al., 2014).

In summary, older adults exhibit deficits in applying grip force that are not simply accountable in terms of neuromuscular activation. Rather they reflect a decreased ability to specify and coordinate motor commands, including those required for prospective (i.e. anticipatory) feedforward control. These age-related changes can be characterized more generally as reversion to immature modes in which muscles are subject to "elementbased" (rather than synergistic) control (Gorniak et al., 2011; Kapur et al., 2010). The consequences of such deficits in muscle coordination include impaired stabilization of the moments produced on hand held objects, and reduced efficiency in translating the forcegenerating capabilities of muscles into motor performance. Their specific nature serves to further emphasise that the requirement to generate maximum grip force places significant demands on CNS functions.

\subsection{Relationships between variations in grip strength and other indices of age-related changes in muscle coordination}

Motor ageing is associated with widespread changes in the central and peripheral nervous systems that encompass glial function (e.g. (Kwan, 2013), neurotrophic signalling (e.g. Ulfhake et al., 2000), neurochemistry (e.g. Darbin, 2012), and the structural (e.g. Seidler et al., 2010) and functional (e.g. D. J. Clark et al., 2011) properties of neurons. In seeking to establish the basis of the relationship between changes in maximum grip strength and manifestations of frailty and cognitive decline, it is instructive to consider patterns of variation that are common between this test and other indices of age-related changes in muscle coordination.

There is a dearth of studies in which changes in grip strength have been related directly to the quality of muscle coordination exhibited in both this and other complex motor tasks. There are however many instances in which grip strength has been assessed in the same context as other measures of motor performance. For older people, maximum grip strength is an independent predictor of the capacity to perform many activities of daily living (ADLs) (KATZ et al., 1963) and instrumental activities of daily living (IADLs) (Lawton and Brody, 1969) including those that have significant demands in terms of muscle coordination (Ensrud et al., 1994; Giampaoli et al., 1999; see also Ling et al., 2010; Taekema et al., 2010). It is also strongly associated with routine activities of daily living that require a high degree of manual dexterity (Hyatt et al., 1990; Incel et al., 2009). Age-related decreases in manual dexterity (assessed in the laboratory) have consistently been associated with declines in maximum grip strength (Figure 9), even though the absolute force requirements of the dexterity assessment tasks are extremely 
low (e.g. Bowden and McNulty, 2013; Marmon et al., 2011; J. A. Martin et al., 2015). By the same token, increases in grip strength, brought about in healthy older individuals by extended (six weeks) periods of training, lead to improvements in hand dexterity that are correlated with the magnitude of the gains in strength (Olafsdottir et al., 2008).

Insert Figure 9 About Here

Declines in manual dexterity and maximum grip strength are both indicative of the deficiencies in muscle coordination experienced by older adults (Barry et al., 2005; Morgan et al., 1994). A variety of degenerative changes, including reductions in the number of corticospinal fibres (Eisen et al., 1996), a decrease in intracortical inhibition (Peinemann et al., 2001), and neuronal degradation in other brain centres (Sjöbeck et al., 1999), impede the ability of older adults to apply maximal force, by limiting the flexibility and adaptability of muscle coordination (Barry and Carson, 2004b). Consistent with this analysis, improvements in force generating capacity brought about by resistance training in this population are mediated by alterations in the spatial and temporal composition of intermuscular synergies (Barry and Carson, 2004a), in a manner that suggests processes akin to motor learning, rather than peripheral adaptations of the type that occur in young adults (Barry et al., 2005).

\section{Relationship between age-related changes in grip strength and cognitive function}

The close associations that have been observed between measures of frailty and cognitive decline have been documented extensively. In this context it has been noted that the elements of the frailty phenotype most closely conjoined with cognitive dysfunction are those (grip strength and timed walk) that primarily reflect the integrity of neural processes required for effective and efficient muscle coordination (e.g. Boyle et al., 2010). The purpose of the present section is not to recapitulate this material, but rather to consider the specific nature of the relationships that exist between maximum grip strength and cognitive function. The outcomes of this analysis do however suggest that the tripartite relationship between grip strength, frailty and cognitive decline, can only be understood by recognising that certain changes in central neurological functions are the common mediating factor.

\subsection{Longitudinal studies using compound assessments of cognitive function}

In the context of longitudinal studies using screening instruments such as the Mini Mental State Examination (MMSE), it has been reported consistently that maximum grip strength measured at the beginning of the assessment period predicts subsequent changes in these multiple domain tests of cognitive function. For example, in a study of more than two thousand non-institutionalised Mexican Americans aged over 65 years, (Alfaro-Acha 
et al., 2006) noted that participants in the lowest quartile of handgrip strength at baseline were more likely to show a decrease in MMSE scores over a 7-year period than participants in the highest quartile (Figure 10). The relationship remained present when the scores were adjusted for age, gender, education, marital status, medical conditions, depression, near and distant vision impairment, and body mass index. Auyeung et al. (Auyeung et al., 2011) reported similar associations between grip strength assessed at baseline and (four years) subsequent changes in MMSE scores (adjusted for age, years of education and baseline MMSE score) (see also Gallucci et al., 2013; Raji et al., 2005; Stijntjes et al., 2016; Taekema et al., 2010). Atkinson et al. (Atkinson et al., 2010) noted an equivalent dependency upon grip strength at baseline (after adjustment for demographics, comorbid conditions, medications, and lifestyle factors), when the Modified Mini-Mental State examination (3MS) was used as a global measure of cognitive function in a six years longitudinal assessment.

Insert Figure 10 About Here

It is furthermore apparent that changes in grip strength over an extended period are predictive of declines in many aspects of cognitive function. In the Swedish Adoption/Twin Study of Aging (SATSA), a battery of tests was used to assess cognitive domains assigned as: verbal abilities, spatial abilities, processing speed, and memory, and grip strength measured, during a longitudinal assessment spanning twenty years. In all four domains, after the age of 65 years, there was a robust longitudinal relationship between changes in grip strength and cognition (Sternäng et al., 2015). Using a composite measure of memory defined by: word recognition; three-item recall; and address recall, Christensen et al. (Christensen et al., 2000) reported that this declined in association with grip strength over the course of a 3.5 years observation period. Focusing on individual variability of performance in four putative cognitive domains (referred to as "crystallized intelligence, speed, memory, and spatial functioning") in the same cohort, Christensen et al. (Christensen et al., 1999) also noted that greater declines in grip strength were associated with increased fluctuation of memory function. Employing a large suite of nineteen cognitive tests to generate a composite measure of global cognitive function, Buchman et al. (Buchman et al., 2014) observed that its rate of change was strongly correlated with that of grip strength, when the tests were performed on an annual basis over a period of six years. Drawing upon data collected from 449 individuals without a diagnosis of dementia who participated in the Origins of Variance in the Oldest-Old: Octogenarian Twins (OCTO-Twin) study, Björk et al. (Praetorius Björk et al., 2016) examined ten tests of cognitive performance representing domains designated semantic memory, episodic memory, spatial ability, motor- and perceptual speed, short-term memory, and working memory. The tests were administered at two years intervals over a ten years period - coded as years before death, in conjunction with measurements of maximum grip strength. It was demonstrated that in four of the six domains (semantic memory, episodic memory, spatial ability, and short-term memory), the rates of decline of cognitive function prior to death were closely associated with the rate of decline of grip strength prior to death. Indeed, with the exception of an investigation that engaged individuals in their ninth decade (Deary et al., 2011), there have been few longitudinal 
studies in which clear associations between maximum grip strength and these measures of cognitive function have not been obtained (see Clouston et al., 2013 \& Rijk et al., 2015 for reviews). The intimacy of this relationship is emphasised by the complementary finding that higher levels of cognitive function predict smaller subsequent declines in grip strength (Cooper et al., 2017; Meincke et al., 2016; Raji et al., 2005; Stijntjes et al., 2016; Taekema et al., 2012) (Figure 11).

Insert Figure 11 About Here

On occasion a factor-analytic approach has been employed to derive factorweighted latent variables from sets of physiological and sensory measurements. In applying this approach to data gathered in the Victoria Longitudinal Study, MacDonald et al. (MacDonald et al., 2004) established that grip strength loaded reliably on a latent variable that accounted for approximately $40 \%$ of the longitudinal variance in five groups of cognitive assessments obtained from 125 adults aged between 67 and 95 years over a twelve year period.

Although the veracity of a construct of Mild Cognitive Impairment (MCI) and its clinical utility remain issues of intense debate (e.g. Lock, 2013), if thus designated by a battery of tests designed to assess global cognitive function and five specific cognitive domains (episodic memory, semantic memory, working memory, perceptual speed, and visuospatial ability), the risk of first occurrence during an observation period of twelve years is associated with the maximum grip strength measured at baseline (Boyle et al., 2010). Using a battery of twenty tests assessing a broad range of cognitive abilities commonly affected by $\mathrm{AD}$, and individual clinical examinations, Buchman et al. (Buchman et al., 2007) examined the relationship between maximum grip strength at baseline and the incidence of subsequent AD classification over an extended follow-up period of (average 5.7 years). Controlling for age, sex and education, a higher level of grip strength was associated with a decreased risk of $\mathrm{AD}$, such that a person in the 10th percentile for grip strength at baseline had a more than twofold greater risk of developing $\mathrm{AD}$ than a person in the $90^{\text {th }}$ percentile at baseline (Buchman et al., 2007). Among 165 individuals enrolled previously in the Rush Memory and Aging Project, grip strength assessed proximate to death was a reliable predictor of AD pathology (after adjusting for level of physical activity, various physical performance measures, and chronic diseases) in individuals with and without a diagnosis of dementia (Buchman et al., 2008). In a cohort of 900 community-based older persons without dementia at the baseline evaluation, who were followed for 3.6 years, clinical diagnoses of $\mathrm{AD}$ were made on the basis of a history of cognitive decline and evidence of impairment in 2 or more domains of cognition, of which memory was one of the domains affected. A proportional hazards analysis indicated a reliable association between maximum grip strength and risk of developing $\mathrm{AD}$ that persisted following adjustment for BMI, physical activity, pulmonary function, vascular risk factors, vascular diseases, and apolipoprotein E4 status (Boyle et al., 2009). Using data from the Study of Dementia in Swedish Twins, Gatz et al. (Gatz et al., 2010) also reported that during a three years observation period, grip strength was a 
reliable predictor of incident AD. Handgrip strength is both a robust predictor of dementia prevalence (van Iersel and Rikkert, 2006), and among those having a clinical classification of MCI, those with lower grip strength are more likely to develop dementia (L. Wang et al., 2006).

\subsection{Cross-sectional studies using compound assessments of cognitive function}

While it may be the case that cohort studies provide a high quality of evidence, there is also an abundance of studies in which a cross-sectional design has been used to examine relationships between maximum grip strength and cognitive function, or in which the cross-sectional correlations (intercepts) derived from longitudinal data have been analysed. For example, the intercepts for the growth curve models applied to data derived from the OCTO-Twin study by Björk et al. (Praetorius Björk et al., 2016), revealed that in individuals aged more than 80 years, maximum grip strength was associated with levels of performance in all of the assessed cognitive domains (semantic memory, episodic memory, spatial ability, motor- and perceptual speed, short-term memory, and working memory). Given the known limitations of cross-sectional studies, circumspection is appropriate. Nonetheless, it is apparent that the outcomes of such investigations are broadly in accordance with those derived from longitudinal studies. With a view to representing this body of knowledge succinctly, it can be helpful to distinguish the testing instruments that were employed, and the cognitive domains that were nominally assessed. Necessarily however, given the lack of consensus that characterises the delineation of theoretical constructs in cognitive science, and the indistinct relationships between these constructs and the vast array of measurement tools that have been used, any notional discrimination between elemental cognitive processes must be approached with caution.

In a study engaging more than eight hundred live-at-home frail elderly individuals of average age 76 years, Shechtman et al. (Shechtman et al., 2004) reported that those designated "cognitively impaired" (MMSE < 24) exhibited the lowest grip strength (see also (Raji et al., 2005; Yassuda et al., 2012). On the basis of a younger (49 to 65 years) sample of almost one thousand people, Malmstrom et al. (Malmstrom et al., 2005) noted the presence of a similar relationship, following adjusting for age, sex, years of formal education, stratum (inner city or suburbs), and the presence of a range of physical and psychological comorbid conditions. Adjusting for age, age-squared, sex, level of education, chronic conditions, and number of medications, Robertson et al. (D. A. Robertson et al., 2014) noted that for a group of more than 4,000 adults aged above 50 (mean 62 years), grip strength was a reliable predictor of "global cognition" - a measure derived from the MMSE and the Montreal Cognitive Assessment (MoCA). The relationship between MMSE scores and grip strength has also been shown to hold in the oldest old (85 and 89 years), with a similar set of covariates having been included (Ling et al., 2010; Taekema et al., 2012).

Equivalent outcomes have been obtained when "pencil and paper" tests other than the MMSE have been employed. In the context of the Honolulu Heart Program/Honolulu-Asia Aging Study, Charles et al. (Charles et al., 2006) highlighted that in a sample of more than 3500 men (aged 71-93 years), maximum grip force was 
inversely associated with scores on the Cognitive Abilities Screening Index Instrument (CASI). When assessed on the basis of AH4 intelligence test scores, the relationship between grip strength and cognitive function evident in an English cohort of more than seven hundred people, was expressed to a greater degree in men than in women (Syddall et al., 2003). Whereas, using the Short Portable Mental Status Questionnaire (SPMSQ) to assess cognitive function in a group of more than three thousand women, Abellan van Kan et al. (Abellan van Kan et al., 2013) reported that SPMSQ scores were clearly associated with grip strength. In the context of a smaller scale study, in which the Mental Test Score (Hodkinson, 1972) was administered, Phillips (Phillips, 1986) obtained a similar relationship between test score and grip strength in female patients admitted acutely to a geriatric ward. Using the Chinese version of the Community Screening Instrument of Dementia (CSI-D) to classify two thousand male and two thousand female individuals as with (CSI-D score $>28.4$ ) or without cognitive impairment, Auyeung et al. (Auyeung et al., 2008) demonstrated that the two groups differed reliably with respect to maximum grip strength. The relationship was independent of muscle mass (assessed using DXA), skeletal mass, age, physical activity levels, and various diagnostic markers of disease.

\subsection{Cognitive processes}

Historically the term executive function has been used in a blanket fashion to encompass a broad range of cognitive processes. The point has been made previously (e.g. Stuss, 2011a; 2011b) that this is a psychological construct lacking a relation to specific anatomical structures or physiological processes. Although new classification schemes have been defined on the basis of neuroanatomical features and aspects of evolutionary development (e.g. Cicerone et al., 2006), in so much as these are often context dependent (i.e. devised for the purposes of addressing specific clinical conditions), they can be unduly restrictive. More generally, it need not be presumed that the brain 'divides up its functions into categories which correspond to our concepts or vocabulary' (Bullock, 1965). For the present purposes therefore, a pragmatic approach is adopted, whereby cross-sectional studies that have been conceived of in terms of relationships between grip strength and specific cognitive processes, are grouped according to such distinctions such as: linguistic/non- linguistic and with/without an explicit memory component. It is beyond contention that these studies all relate to aspects of behaviour embraced by the term cognition.

\subsubsection{Linguistic tasks without an explicit memory component}

Using a task that required the deduction of the letter in a sequence that would continue an established pattern, MacDonald et al. (MacDonald et al., 2011) determined that there was a clear time varying association between performance on this task and maximum grip force, when community dwelling adults aged over 65 years were tracked over a period of six years. In the context of the Swedish Adoption/Twin Study of Aging, Sternäng et al. (Sternäng et al., 2015) employed the Information Subtest (from the Wechsler Adult Intelligence Scale-Revised [WAIS-R]; (Wechsler, 1981), Synonyms, and Analogies, and determined that there was an overall effect of grip strength on this 
measure at 65 years of age, and that changes in grip strength over a monitoring period of twenty years were associated with changes in Information Subtest scores (Figure 12). Employing a variant of the Symbol Digit Modalities Test - that requires as many letterdigit combinations as possible be generated within a fixed time period, Taekema et al. (Taekema et al., 2012) noted an association between level of performance and grip strength at age 85 and 89 years (following adjustment for various factors - sex, height, weight and for income, education, prescription medication). In large cohorts of noninstitutionalized middle-aged (49 to 65) African Americans (Malmstrom et al., 2005) and European citizens aged 50 years or older (Aichberger et al., 2010), verbal fluency (ability to name as many different animals as possible in $60 \mathrm{~s}$ ) is reliably associated with grip strength (following adjustment for a wide range of variables such as sociodemographic profile, comorbid conditions, mental-health status, and BMI). This is also the case with respect to general tests of mental ability (i.e. the Moray House Test) comprising items that predominantly require verbal reasoning (Deary et al., 2006).

Insert Figure 12 About Here

\subsubsection{Linguistic tasks with an explicit memory component}

In the delayed recall (DR) test, a list of 10 short, concrete, high frequency nouns is read aloud. After an interval of typically $5 \mathrm{~min}$, the participant is asked to recall the items. Aichberger et al. (Aichberger et al., 2010) reported that in a sample comprising 17,710 persons (aged 66.6 years at follow-up) located in 11 European countries, both at baseline and when reassessed 2.5 years later, grip strength was reliably associated with DR test performance. Kuh et al. (Kuh et al., 2009) presented similar findings for a group of more than one thousand men, who completed a task that required distinct sets of 15 words to be learned in three separate trials - demonstrating a reliable association with grip strength when performance was assessed at 43 and 53 years of age.

\subsubsection{Non-linguistic tasks without an explicit memory component}

Part A of the Trail Making Test consists of 25 numbered circles distributed over a sheet of paper. The participant is instructed to connect the circles ascending order, as quickly as possible, without lifting the pen or pencil from the paper. McGough et al. (McGough et al., 2013) reported that in a group of 201 older adults with amnestic mild cognitive impairment (aMCI), the performance of this test was reliably associated with grip strength (after adjusting for age, sex and age-related factors). Examining 708 people drawn from the Swedish Adoption/Twin Study of Aging over a 20 years period, Sternäng et al. (Sternäng et al., 2015) observed an overall effect of grip strength on a "processing speed" measure derived from Symbol Digit and Figure Identification tasks, and on a "spatial ability" score generated from tests including Figure Logic, Block Design (Wechsler Adult Intelligence Scale - Revised [WAIS-R]), and Card Rotation. Furthermore, decreases in grip strength over the duration of the study were associated with declines in both the "processing speed" measure and the "spatial ability" score. 
Deary et al. (Deary et al., 2011) noted that test scores for Raven's standard progressive matrices - which is considered to be a relatively pure test of inductive reasoning or "fluid intelligence", were reliably associated with grip strength in 207 individuals drawn from the Lothian Birth Cohort 1921 when these were assessed at age 79, 83, and 87. Notably, although the Raven score intercept was associated with a Moray House Test score obtained at age 11, there was there was no evident relationship between the childhood test score and grip strength in old age.

\subsubsection{Non- linguistic tasks with an explicit memory component}

In the 12-Picture Learning Test, twelve pictures are successively presented at a rate of one every two seconds. The participant is then asked to recall as many pictures as possible. The procedure is typically carried out three times to yield a measure of immediate recall. After 20 minutes, a delayed recall test is conducted. Taekema et al. (Taekema et al., 2012) reported that both immediate and delayed recall performance was associated with grip strength when assessments were made at age 85 and 89 years (following adjustment for sex, height, weight and for income, education, prescription medication). Similarly, Sternäng et al. (Sternäng et al., 2015) noted a reliable influence of grip strength on a compound measure that incorporated Thurstone's Picture Memory Task and the Digit Span test of the WAIS-R. Reductions in grip strength after the age of 65 years were also reliably associated with declines in the performance of these tests. In the computation span test (Salthouse and Babcock, 1991) arithmetic problems are presented orally at a normal rate of speaking, with the participant choosing the correct answer from three alternatives. The final digit from each problem is to be recalled subsequently. Over the course of several trials the number of problems in each sequence is increased. Community dwelling adults aged over 65 years drawn from the Victoria Longitudinal Study, who were tracked over a period of six years, exhibited declines in computation span that were linked reliably to decreases in grip strength. A directly corresponding pattern of covariation with grip strength was manifest for recall tests of global facts drawn from the domains of science, history, art, sports, and geography (MacDonald et al., 2011).

\section{Common mediation by central neurological functions}

It is evident that if evaluated in the context of both longitudinal and cross-sectional cohorts of older adults, there is clear and pervasive relationship between maximum grip strength and every measured expression of cognitive function. When assessed throughout the lifespan, the more rapid the decrease in grip strength over time, the larger the associated decline in cognitive function. The influence of peripheral factors such as muscle mass and fibre type that have significant bearing upon individual capacity notwithstanding, in people of a similar age, those who have greater grip strength tend to exhibit higher levels of cognitive function. In short, "People who grip better, think better" (Deary et al., 2011, p. 699). In light of the demands on CNS functions that are imposed 
by the requirement to generate maximum grip force, and in view of the intimacy of the relationship between variations in grip strength and cognition, the point is further accentuated that changes in central neurological processes are the common mediating factor. While our knowledge in this regard remains incomplete and constrained by poor understanding of the brain's native ontology (Anderson, 2015), it is now possible to resolve some of these processes, and to distinguish defining features.

\subsection{Shared neural substrates}

For most of its history, the hypothesis that the evolution of motor systems provided a basis for the emergence of cognitive function (e.g. Calvin, 1986) resisted empirical examination. With the advent of modern neuroimaging and comparative anatomical techniques however, it has proved to be a fertile source of inspiration for contemporary neuroscience. As a direct consequence, it is becoming increasingly well established that common regions in the brain are engaged in both motor tasks and those typically classified as cognitive/executive (Cremen and Carson, 2017).

\subsubsection{Response Inhibition}

The Eriksen Flanker Task is emblematic of a class of response inhibition tests used in cognitive psychology to assess the ability to suppress responses that are inappropriate in a particular context. A number of studies now suggest that the presupplementary (preSMA) and supplementary motor areas (SMA) -elements of the cortical motor network (e.g. Mendoza and Merchant, 2014), play an active role in resolving the response conflicts engendered by this family of tasks (Taylor et al., 2007; Zurawska Vel Grajewska et al., 2011). In this regard, pre-SMA forms part of a circuit that also encompasses dorsolateral prefrontal cortex, right inferior frontal cortex, and structures in the basal ganglia (Richard Ridderinkhof et al., 2011). In variants of the Simon task, activity in these regions in presence of errors has been related to the driving of attention towards task-relevant perceptual information (Danielmeier et al., 2011). While individual differences in pre-SMA grey-matter density are closely associated with levels of capability in conflict resolution paradigms (van Gaal et al., 2011), related variations in SMA grey-matter volume also correlate with the performance of other tasks used to assess executive function (Sakai et al., 2012). Although delineation of the boundaries of inferior frontal regions engaged in cognitive control remains a matter of debate, it is evident that its posterior part encompasses ventral premotor cortex (PMv). On a probabilistic basis, brain imaging registered activity in this part of the brain during response inhibition tests, set-shifting paradigms and motor control tasks cannot be reliably distinguished (Neubert et al., 2013). Neural correlates of the decision making process engendered by response inhibition tests (i.e. the Eriksen Flanker Task) can also be delineated in primary motor cortex (Grent-'t-Jong et al., 2013; P.-A. Klein et al., 2014; 
Michelet et al., 2010; Verleger et al., 2009). Indeed, it has been proposed that higher order executive control might be an evolutionary extension of the frontal cortex-basal ganglia loops that guide resolution of (motor) response conflict, such that the role of these neuronal mechanisms extends to a range of processes including the reorienting of attention and the updating of working memory (Neubert et al., 2013).

\subsubsection{Working Memory}

There exists an extensive corpus of work conducted with non-human primates, demonstrating that the detection of task-relevant somatosensory information engages primary sensory, prefrontal, premotor and motor cortices. If the experimental paradigm demands that the characteristics of this sensory input remain accessible as a basis for subsequent comparison (aka "working memory"), prefrontal and premotor areas of the frontal lobe are engaged. Should presentation of a further sensory stimulus follow, and the animal is required to make a binary decision (i.e. higher or lower) concerning some shared attribute (e.g. frequency), activity related to this determination is observed in prefrontal, premotor, parietal, and primary motor cortices. This occurs regardless of whether the decision is immediate or postponed, and in a fashion that cannot be accounted for by the motor demands of the task (see Romo and de Lafuente, 2013 for review). Analogous evidence supporting the view that the cognitive decision-making process is widely distributed through the cortex, and encompasses elements of the classical motor network, has also been obtained in humans. Using an experimental paradigm equivalent to that employed in the animal studies, Preuschhof et al. (Preuschhof et al., 2006) showed that the BOLD fMRI delineated brain activity registered during maintenance of the memory trace included the ventrolateral prefrontal cortex (VLPFC), and the medial (PMm) and ventral premotor (PMv) cortex - the latter areas both playing an integral role in relation to motor behavior. Increased activity was registered in lateral $\mathrm{PFC}$, and in medial and lateral PMC during the decision-making phase, and in M1 and in SMA during initial encoding. It has been shown separately that the integration of prior information during the encoding phase also gives rise to additional activity in PMv (Preuschhof et al., 2010). Beyond the patterns of brain activity that are present during such tasks, improvements in somatosensory discrimination that occur over the course of training are associated with changes in the functional connectivity of frontal motor areas. These remain apparent following removal of effects that are correlated with somatosensory activity per se. Specifically, perceptual training induces bilateral changes in the functional connectivity of brain networks including PMv and bilateral SMA and M1, and in links between SMA and M1, PMd, and cerebellar cortex (Vahdat et al., 2014). In a recent Activation Likelihood Estimation (ALE) meta-analysis resolving core elements of the brain network engaged during perceptual decision-making tasks, Keuken et al. (Keuken et al., 2014) determined that as the difficulty of the task was elevated, significant activation clusters were obtained bilaterally within the region of the precentral gyrus (aka the motor strip). A broadly similar picture derived from the study of classic motor signatures has emerged from the use of other brain imaging modalities such as electroencephaolography (EEG) and magnetoencephalography (MEG) (e.g. de Lange et al., 2013). This extends beyond decisions based on somatosensory information to 
encompass visual motion (Donner et al., 2009; 2007) and value-based choices (e.g. Gluth et al., 2013; see also Gluth et al., 2012). Thus while it is apparent that regions such as dorsolateral prefrontal cortex (dlPFC) - typically conceived of as an obligatory element of the cortical network engaged during tasks that require the discrimination and subsequent comparison of sensory information (Heekeren et al., 2006), play an important role, the balance of evidence derived from human neuroimaging indicates that elements of the cortical motor network are also recruited in maintaining the related percepts in working memory, and in computations governing the decision process (Siegel et al., 2011).

Wayne Kirchner introduced the n-back task in 1958 (Kirchner, 1958). In spite of weak convergence with other measures of working memory (Kane et al., 2007), the task and its variants (e.g. Jaeggi et al., 2003) are paradigmatic in both clinical and experimental settings. In two Activation likelihood estimation (ALE) meta-analyses published together, Owen et al. (Owen et al., 2005) and Glahn et al. (Glahn et al., 2005), twelve areas of activation emerged. The subset of only five areas for which there was a corresponding response included Brodmann area 6 (BA6) -typically considered a motor area (Uttal, 2013). In a comparison of seven further meta-analyses (in addition to the two that used the n-back procedure), Uttal noted that forty-seven Brodmann areas were reported as being activated during working memory tasks (i.e. across the nine metaanalyses). Of these forty-seven brain regions, only Brodmann area 6 was designated as being activated in every case. Such meta-meta-analyses further emphasise that for tasks that notionally sample working memory, and in the context of extremely large variations in regional brain activation, the detection of signal in cortical motor areas is one of the most robust findings (e.g. Niendam et al., 2012).

\subsubsection{Perception-Attention Tasks}

The serial reaction time task (SRTT) is typically considered to engage processes supporting the temporal organization of behavior, the formation of high-order associations, and the prediction of future events, and thus to reflect the principles that govern learning and memory (E. M. Robertson, 2007). Conceived of as a perceptual counterpart to the SRTT, the serial prediction task (SPT) (Schubotz, 1999) requires the extraction and prediction of repetitive sensory patterns within sequentially presented series of (visual) stimuli. Importantly, there is no motor co-production, and the stimuli are not given any explicit motor significance. Performance is assessed subsequently by means of a forced judgment concerning the incidence of a sequential violation. In a series of studies employing $\mathrm{fMRI}$ it has been demonstrated that this paradigm robustly engages the cortical motor network, particularly premotor and associated parietal areas (Schubotz and Cramon, 2002a; 2001a; Schubotz and Yves von Cramon, 2002). The activity also extends to primary motor cortex, the cerebellum and basal ganglia (Schubotz and Cramon, 2001b). Corresponding findings are obtained when the stimuli are in the auditory domain (Schubotz and Cramon, 2002b; Schubotz et al., 2003). Indeed, when assessed on the basis of extended collections of studies in the context of meta-analyses, it is further emphasised that in perceptual-attentional tasks - even those that do not call for either the execution or 
the imagination of movement, the activation of premotor cortex is a cardinal feature (Schubotz, 2007; 2004; Schubotz and Cramon, 2003).

\subsubsection{Tests of Cognitive Flexibility}

Trail Making Tests (TSTs) are variously described as measuring processing speed, sequencing, mental flexibility (Bowie and Harvey, 2006), visual search, scanning, and executive functions (Tombaugh, 2004). In the first part of the test (TMT-A), lines are drawn sequentially in order to connect 25 encircled numbers distributed on a sheet of paper. In the second part (TMT-B), the requirements are similar, with exception that the individual being tested must alternate between letters and numbers (e.g., 1, A, 2, B, 3, C, etc.). When patterns of association with the levels of performance exhibited on other neuropsychological tests are analysed, it has been surmised that the TMT-A draws mainly upon visuoperceptual abilities, that the TMT-B is primarily an expression of working memory and task-switching ability, and that B-A difference scores are a "relatively pure indicator of executive control abilities" (Sánchez-Cubillo et al., 2009). The TMT is also used extensively in clinical contexts with a view to detecting frontal executive dysfunction (Chan et al., 2015). It is notable therefore that when variants of the TMT (with the same visual and motor demands) specifically adapted for neuroimaging are employed, the contrast between the TMT-A and TMT-B reveals differences in BOLD response in the dorsal part of M1 (Jacobson et al., 2011; see also Zakzanis et al., 2005), consistent with previous observations that activity in this brain region varies in a nearly linear fashion with working memory load (Tsukiura et al., 2001; Zarahn et al., 2005). Using a fMRI optimized version of the TST-B, Allen et al. (M. D. Allen et al., 2011) also noted the presence of task-related activation in the left precentral gyrus (M1), bilateral premotor cortex, and the medial pre-supplementary motor area (see also Moll et al., 2002). When measured using functional near-infrared spectroscopy, bilateral activity is detected during the TMT-B task in premotor regions (in addition to ventro- and dorsolateral prefrontal cortex) (Müller et al., 2014). In a further study using the same imaging methodology, activity was also detected in primary motor cortex, with older participants exhibiting greater task related changes in $\mathrm{O}^{2} \mathrm{Hb}$ signal strength in this brain region and in the right premotor cortex in both forms of the test (Hagen et al., 2014). Employing EEG-derived measures, Wölwer et al. (Wölwer et al., 2012) reported associations between TMT-B task performance and the current density of M1 designated sources.

\subsubsection{Numerical Cognition}

The more general difficulties (e.g. Uttal, 2001) that arise in seeking to develop a taxonomy of cognitive processes notwithstanding, the triple code model (TCM) of numerical cognition (e.g., Dehaene and Cohen, 1997; 1995; Dehaene et al., 2003) has informed contemporary attempts to identify an anatomo-functional system subserving the processing of numbers and mental calculation. The TCM model encapsulates (i) a (bihemispheric) numerical magnitude representation associated with the intraparietal sulcus 
(IPS); (ii) a verbal representation of numbers associated with left perisylvian language areas and the left angular gyrus (AG); and (iii) a visual number form representation involved in the recognition of Arabic digits and associated with bilateral fusiform and lingual regions. And yet there is persuasive evidence that neural circuits nominally classified as "motor" also assume functional relevance in the domain of numerical cognition. In a series of initial investigations, often conducted using PET, differential (i.e. specific task-related) activity in the (left) precentral gyrus (i.e. the motor strip), along with the (left) intraparietal sulcus, was noted repeatedly during the retrieval of mathematical facts (e.g. $2 \times 4$ ) (Zago et al., 2001), additions (Pesenti et al., 2000; Venkatraman et al., 2005), and multiplications (Dehaene et al., 1996; Zago et al., 2001). Bilateral activity in the precentral gyrus has been reported for numerical judgements of relative magnitude (Liu et al., 2006) and for subtractions (Rueckert et al., 1996). In studies (typically using fMRI) that have distinguished Brodmann area 4 (primary motor cortex) from Brodmann area 6 (PMC and medially SMA) - both of which occupy portions of the precentral gyrus, bilateral activity is registered in premotor cortex during subtractions (Rueckert et al., 1996). When performing additions, engagement of left premotor cortex and SMA has been registered (B. M. de Jong et al., 1996; E. Klein et al., 2014). In summarising the outcomes of ten studies that evaluated patterns of functional and "effective" brain connectivity associated with numerical cognition, Moeller et al. (Moeller et al., 2015) noted that the task relevant networks are widespread, extending beyond (intra) parietal and (pre) frontal cortex to encapsulate the precentral gyrus. A role for bilateral SMA in the processing of number magnitude is also suggested by analysis of white matter structural connectivity (E. Klein et al., 2014). This overall pattern of observations is consistent with the hypothesis that brain regions that play an obligatory role in the planning and execution of finger movements are also central to the representation and manipulation of numbers (Anderson, 2010; Anderson and PennerWilger, 2013; Penner-Wilger and Anderson, 2013).

\subsubsection{Object Knowledge}

In the context of the hypothesis that simulation is a basic brain operation that supports a broad spectrum of cognitive processes, it has been proposed that procedural knowledge is central to the formation of object concepts (e.g. Barsalou, 2009). As a corollary, it is predicted that brain regions engaged during the planning of actions also play a necessary role in the encoding that mediates a recollection of objects and events (Beauchamp and A. Martin, 2007; A. Martin, 2007). There is now a good deal of empirical evidence in support of the supposition that retrieval of the properties associated with an object's appearance engenders activity within the motor (and sensory) networks that were active during the acquisition of this information. For example, Weisberg et al. (Weisberg et al., 2007) asked individuals to match pictures of objects never encountered previously, both prior to and following training during which they were required to use these novel objects as tools to perform specific actions. Following the completion of training, visual object identification was associated with (BOLD fMRI) activity registered in left dorsal premotor cortex and intraparietal sulcus - areas that were active during manipulation of the objects. 
The engagement of various elements of the motor network (i.e. motor and premotor cortex, basal ganglia, and cerebellum) is not restricted to the identification of objects. It also occurs during the mental rotation of abstract objects. In an influential study employing Shepard and Metzler's (Shepard and Metzler, 1971) mental rotation task - a classic assessment of dynamic imagery, Richter et al. (Richter et al., 2000) reported bilateral fMRI registered activity in the lateral premotor and supplementary areas that appeared directly related to the act of mental rotation. This pattern of observations has been repeated on a number of occasions using protocols employing abstract objects, and concrete items such as tools (Vingerhoets et al., 2002). Furthermore, it is now apparent that the recruitment of dorsolateral premotor areas cannot simply be accounted for in terms of the processing of spatial orientation information. Rather, it relates to mental rotation in a fashion that suggests a more general role in mediating the distribution of visuo-spatial attention (Lamm et al., 2007). This is consistent with the findings derived from the use of fMRI (e.g. Simon et al., 2002; Tanaka et al., 2005), PET (e.g. Hanakawa et al., 2002) and EEG (e.g. Brovelli et al., 2005), that the dorsolateral premotor cortex plays an instrumental role in other tasks designed to assess spatial attention and spatial working memory. The balance of available evidence suggests that activity in SMA during mental rotation tasks is at least as conspicuous (see Zacks, 2008 for a meta-analysis). While the involvement of parietal regions is also an obligatory aspect of the neural processing that mediates the performance of these tests, examination of the spatialtemporal evolution of the corresponding brain dynamics reveals that BOLD registered activity in SMA and premotor areas discriminates the nature of the cognitive event prior to that recorded from parietal cortex (Mourao-Miranda et al., 2009, see also Sasaoka et al., 2014).

\subsubsection{Language}

Assuming particular prominence in relation to the general theory that the emergence of cognitive faculties was driven by exaptation - the exploitation of existing neural resources that had evolved to serve a specific motoric purpose, is the supposition that there exists a common brain architecture for language and certain key aspects of motor control (e.g. Steele et al., 2012). In providing a source of evidence that complements and extends inferences drawn on the basis of paleoneurological work on hominin fossils and the archaeological record of tool use and manufacture, with respect to this issue more so than any other, modern neuroimaging techniques have permitted the development and evaluation of coherent and physiologically grounded hypotheses (e.g. Hopkins et al., 2012). The scope of this field of enquiry is sufficiently large that it will suffice to note only those findings that are currently beyond reasonable doubt. While it is natural to accept that the human motor system participates in the production of language, it has for some time also been apparent that Broca's area plays a role in receptive language function, the perception of music, and working memory (Amunts et al., 2010; Poeppel et al., 2012). While the mining of large neuroimaging data sets suggests that Broca's area should no longer simply be designated a "language" region (Poldrack, 2006), such analytic approaches have similarly made apparent the engagement of primary motor (Anderson and Penner-Wilger, 2013) and premotor (Rosselli et al., 2015) cortex in 
various facets of language function. These findings are complemented by further metaanalyses that reveal consistent engagement of primary motor (Jirak et al., 2010; Yang and Shu, 2016) and premotor cortex (e.g. Ferstl et al., 2008; Jirak et al., 2010; Price, 2012; Rapp et al., 2012) in mediating components of language that are not directly related to action. While the precise interpretation of such evidence remains a matter of discussion, it can be reconciled with explanatory schemes that emphasise the predictive nature of the motor system in contributing to the perception of speech sounds (e.g. D'Ausilio et al., 2009), and the role of audio-motor mappings in the long term retention of language and other pronounceable sounds (Schulze et al., 2012). It is also consistent with the broader perspective that motor circuits contribute to many facets of language, including the comprehension of phonemes, semantic categories and grammar (Pulvermüller and Fadiga, 2010), and support other aspects of speech processing including the control of conversation (Scott et al., 2009).

\subsubsection{Timing}

In relation to the putative evolutionary pressures that may have promoted the emergence of language - among other hallmarks of human cognition, the argument has been made that an evolved neural organization that permitted objects to be thrown with great force and precision, may also have supported oral-facial motor sequencing, phonemic competence and the emergence of syntax (Calvin, 1994; 1983). The tenet is that neural circuitry having been subjected to strong selection acting upon the proficiency of motor control and coordination, was co-opted to support language functions. More specifically, brain circuits that control the precise and anticipatory timing of muscle activation sequences necessary for adaptive movements, provide the substrate for timerelated functions within the broader domain of cognition (Calvin, 1983). A wealth of contemporary evidence has now emerged to support this assertion, demonstrating that these circuits are engaged not only in the perception of time, but also in related facets of executive function.

As there have been numerous studies reporting functional neuroimaging data in association with the performance of reasonably well-standardised time perception tasks, their outcomes are amenable to summary using "voxel-wise" meta-analysis (e.g. activation likelihood estimate (ALE), Seed-based $d$ Mapping (SDM)). The general picture to emerge is that task-related activity ascribed to Brodmann area 6 is a characteristic feature of temporal cognition (Ortuño et al., 2011; Wiener et al., 2010). Wiener et al. (Wiener et al., 2010) differentiated activity in SMA from that registered in other parts of Brodmann area 6 (BA6). On the basis of eight experiments, they concluded that sub-second perceptual timing reliably engages SMA and other parts of BA6 bilaterally. Considering seven studies that examined supra-second perceptual timing, bilateral activity was reliably evident in SMA, and in BA4 i.e. the primary motor cortex. Using a label-based meta-analysis (i.e. enumerating activation in specific regions of interest (ROIs)), Penney and Vaitilingam (Penney and Vaitilingam, 2008) noted that the presence of activity in SMA (and pre-SMA) and premotor cortex was commonly reported in sub-second timing tasks. Adopting a similar analytic approach, Lewis and Miall 
(Lewis and Miall, 2003) delineated bilateral SMA and premotor activity (along with right cerebellum and left basal ganglia) during timing tasks requiring covert decisions, memory encoding, memory rehearsal of rhythms, or detection of oddballs. The ubiquity and reliability of SMA engagement in particular is such as to suggest that this brain region (along with the right inferior frontal gyrus (IFG)) is integral to the core network mediating time perception (Wiener et al., 2010). Indeed, beyond the integral functional role assumed by this region in the domain of action, the connectivity of SMA promotes many facets of perception and cognition (Narayana et al., 2012). Notably in this regard, brain regions that are particularly active during time estimation - especially those lying within BA6, are recruited when there are increases in the difficulty of working memory and executive function tasks (Radua et al., 2014). It is clear too however, that parts of the cerebellum and components of the basal ganglia (e.g. caudate nucleus and putamen) are implicated both in perceptual timing (Press and Cook, 2015; Wiener et al., 2010) and many other cognitive functions.

\subsection{Focus on the Cerebellum}

In light of persuasive arguments to the effect that a disproportionate and speciesspecific enlargement of the cerebral neocortex over the course of human evolution gave rise to unique cognitive faculties (e.g. Kirkcaldie and Kitchener, 2007; Rakic, 2009), there is an understandable tendency to focus on brain regions therein when investigating the neural circuits that mediate our distinctive mental capabilities (Barton, 2012). It has long been recognized that the cerebellum plays a key role not only in controlling and regulating movement, but also in facets of perception (Baumann et al., 2015) and learning for which the precise representation of temporal information is required (Ivry, 1997). It is now becoming increasingly apparent that the cerebellum was also subject to a rapid increase in size during the course of human evolution. Indeed, it has been proposed that it was the specialisation of cerebellar circuits subserving sensory-motor control and learning that provided preadaptation for language (Barton and Venditti, 2014). In line with the view that a distributed brain network supports cognitive functions across a range of domains (e.g. "flexibility", "inhibition", "working memory", "initiation", "planning" and "vigilance) that are typically treated as distinct, large scale meta-analyses (e.g. Niendam et al., 2012) are consistent in noting that activation in cerebellum (along with other subcortical structures including thalamus, caudate, and putamen) is a pervasive feature. In the context of meta-analyses that restrict attention to the cerebellum however, regional differences in activation can be detected for sensorimotor processing on the one hand, and performing cognitive and "emotional" tasks on the other (Stoodley and Schmahmann, 2009). As with any brain region demarcated along neuroanatomical lines, there are hazards in conceiving of the cerebellum as having a uniform cyto- architecture (Cerminara et al., 2015), and variations in cerebellar cortical anatomy and physiology may contribute to regional differences in function (Keren-Happuch et al., 2014). Nonetheless, it is widely held that spatial differentiation of cerebellar functional activity is due largely to differences in afferent and efferent connectivity (e.g. D'Angelo and Casali, 2012; Ramnani, 2006; Stoodley, 2012). This is in accord with the view that circuits in the cerebellum play a generic role in the formulation of internal models that 
are integral to both motor and cognitive functions (Ito, 2008). More particularly, such models may be crucial not only in the anticipatory feedforward control that is a requirement for adaptive movement, but also when organising behaviour to achieve future goals; in formulating or parsing language; or engaging in social interactions that are based on projecting the actions of others (G. Allen et al., 1997; Imamizu and Kawato, 2009). In line with the assumption that equivalent computations are performed throughout the cerebellum (Ito, 1993), it has therefore been argued that age-related degeneration or other challenges to its integrity, will have directly corresponding (negative) implications for anticipatory planning and coordination, both in the context of movement and cognition (Stoodley, 2012).

When assessed in the context of cross sectional studies, it is increasingly apparent that in older adults, imaging derived markers of cerebellar morphology and connectivity exhibit close associations with a range of indices of cognitive function. For example, the volume of the cerebellar hemispheres is correlated with the performance of verbal and non-verbal working memory tasks (Raz et al., 2000), and with the strength of associative learning (Woodruff-Pak et al., 2001). The volume of the cerebellar vermis is also a reliable predictor of the aspects of cognitive function captured by tests of processing speed, memory, and visual reproduction (MacLullich et al., 2004; T. D. Miller et al., 2013). Voxel based morphometry suggests the presence in older adults of relationships between cerebellar grey matter and "intelligence" measured on general scales such as the WAIS (J.-Y. Lee et al., 2005), and with levels of performance on tests of short and long term memory, non-verbal reasoning, and speed of processing (Hogan et al., 2011). In older individuals (circa 65 years) the "strength" of (resting state fMRI derived) indices of functional connectivity between the cerebellum and the medial frontal gyrus, and between the cerebellum and both the precuneus and posterior cingulate cortex, are predictive of working memory task performance (Bernard et al., 2013). On the basis of the assumption that degenerative changes in cerebellar morphology and connectivity will necessarily compromise existing internal models and impede the formation of new internal models, with consequential effect upon all aspects of prospective control, it has been argued that cerebellar degeneration may account for a significant portion of the cognitive and motor deficits that are a characteristic feature of ageing (Bernard and Seidler, 2014).

\subsection{Focus on the Basal Ganglia}

A group of structures located at the base of the forebrain, the basal ganglia (in the strict classical sense) comprising the dorsal striatum i.e. caudate nucleus-putamen, and globus pallidus, play a prominent role in regulating the coordination of movement. As part of a functional network that encompasses the subthalamic nucleus, substantia nigra, and ventral striatum, it is increasingly appreciated that the basal ganglia also mediate various aspects of cognition (e.g. Leisman and Melillo, 2013; Middleton, 2003; Middleton and Strick, 2000a). While the structures of the basal ganglia comprise important subdivisions and groupings, the general premise is that in terms of function, they subserve the same roles for cognition as they do for movement (Koziol and Budding, 2009). 
As there are authoritative and comprehensive reviews dealing with the anatomy and functions of the basal ganglia (e.g. Koziol and Budding, 2009; Middleton and Strick, $2000 \mathrm{~b}$ ), the information provided here is simply a summary pertinent to the present piece that draws in part upon these previous works. By virtue of a network of connections constituting a cortical-striatal-pallidal-thalamic-cortical system, the basal ganglia have means to modulate activity in multiple cortical regions. Indeed, these nuclei exhibit reciprocal connectivity with all cortical areas. As is the case for the cerebellum, it is differences in the afferent and efferent connectivity of separate channels of the basal ganglia circuitry (Postuma and Dagher, 2006; Robinson et al., 2012) that appears to account for the spatial separation of activity in areas engaged differentially during motor, cognitive, and affective behaviour (Koziol and Budding, 2009). In general however, these nuclei can be conceived of as comprising: input structures, intermediate structures; and output structures, consistent with an evolved role in mediating attention and action selection in sensory and motor systems. In the context of phylogenetically recent neocortical-basal ganglia circuits, they may be conceived of as an interface between subcortical and cortical systems, with the capacity to influence all aspects of behavioral selection (McHaffie et al., 2005), by selectively gating the operations that are performed by the neocortex (Frank et al., 2001; Houk et al., 2007; Stocco et al., 2010). For example, in view of a mediating role for the basal ganglia in various expressions of learning and memory, it has been proposed that circuits within these nuclei serve to "train" connections between posterior cortical areas and frontal cortical regions, as automaticity of behaviour emerges through training (e.g. Frank et al., 2001; Graybiel, 1997; Hélie et al., 2015; E. K. Miller, 2013). These conceptual models are accompanied by a wealth of empirical evidence, collated via meta-analyses, indicating consistent basal ganglia involvement in novelty detection (Kim, 2013) and declarative memory (Scimeca and Badre, 2012; Spaniol et al., 2009). Widespread activity is also typically registered in the basal ganglia during ("working memory) tasks that require the encoding, storing, manipulating and retrieval of information (Arsalidou et al., 2013; Rottschy et al., 2012), and during procedures (e.g. planning and task switching) to which the label "executive functioning" has been applied (Arsalidou et al., 2013).

While we are accustomed to their expression in terms of disorders of movement, diseases of the basal ganglia have a profound impact on cognition. Indeed, although the most readily apparent symptoms of Parkinson's disease (PD) and Huntington's disease (HD) - the two most common neurological disorders of the basal ganglia, are motoric, both first give rise to psychiatric and cognitive syndromes (Aarsland et al., 2009). Within the cognitive domain, these include deficiencies in working memory, attention and other aspects of executive function (e.g. Lawrence et al., 1998; 1996; Owen et al., 1997; 1992; Pagonabarraga and Kulisevsky, 2012). Of particular importance in the present context is the emerging recognition that basal ganglia dysfunction is a crucial factor in the expression of cognitive symptoms in neurodegenerative disease (O'Callaghan et al., 2014). Indeed, atrophy of the striatum and its substructures (as indexed by grey matter volume) is closely associated with age-related cognitive decline (and the occurrence of dementia), in a manner that is typically independent of markers such as hippocampal volume (L. W. de Jong et al., 2012; Hedden et al., 2016; Pini et al., 2016). In summary, disruption of the cortical-striatal-pallidal-thalamic-cortical system arising from disease or degeneration of circuits within the basal ganglia, has not only an exemplary impact on 
motor control, but also profound negative implications for many aspects of cognitive function (Leisman and Melillo, 2013).

As the foregoing presentations make clear, with the benefit of modern neuroimaging methods, it is possible to detect regions in the cerebellum and basal ganglia that exhibit a low degree of spatial overlap when defined in terms of activation thresholds during the execution of "cognitive" and "motor" tasks respectively. It is important to bear in mind however, that all behaviour (i.e. regardless of whether labeled as "cognitive" or "motor") is mediated by distributed neural systems. While these may encapsulate separate anatomic regions or divisions that are spatially circumscribed, and which in some cases exhibit unique cyto-architectural features, they are nonetheless linked in a functionally specific manner (Schmahmann and Pandya, 2014). This characterisation applies with equal force to both subcortical and cortical areas. As a general consequence, degeneration or damage of the basal ganglia or cerebellum (or indeed the thalamus) may produce the same spectrum of cognitive and motor syndromes as those associated with disorders that affect different parts of the cerebral cortex (Graff-Radford et al., 2017). The key point in this regard is that, even in circumstances in which brain damage/degeneration is restricted to territory within subcortical divisions demarcated "motor" or "cognitive", the extensive and distributed nature of the related neural networks dictate that the consequential impairments of behaviour will rarely respect this distinction. In addition, as in many cases these divisions share the same blood supply, they are equally susceptible to microvascular damage and to the impact of the various vascular risk factors associated with ageing.

\section{Age-related changes in brain integrity associated with variations in maximum grip strength}

As the evidence presented in the preceding sections makes clear, with respect to the facets of cognition that have been studied using brain imaging methodologies, taskrelated activity is always observed in brain regions (i.e. constituting the classical motor network) that play an obligatory role in the production of coordinated motor output. This phenomenon can be studied in another way - by selecting a specific region of interest (ROI) within the motor network, and looking across various aspects of cognition to ascertain the scope of its task-related engagement (e.g. Hoffstaedter et al., 2014). In an approach of this type, Penner-Wilger and Anderson (Penner-Wilger and Anderson, 2011) analysed sixty-five studies that reported reliable (i.e. post-subtraction relative to control) activation within the left precentral gyrus (eighty subtractions in total). Of these, eleven were within the domain of "action", two within "emotion", seven within "perception", and sixty within "cognition". In relation to the last, twenty were classified as being in the sub-domain of "attention"; twenty in "memory" (10 in working memory and 7 in explicit memory); sixteen in "language" ( 1 in orthography, 2 in phonology, 9 in semantics, and 4 in speech); two in "mathematics", one in "theory of mind"; and one in "time". The point has also been made that attempts to locate cognitive processes only in specific areas in the human brain do not accord with the characteristics of neurodegenerative conditions in which cellular changes, inclusion bodies, atrophy, hypoperfusion or hypometabolism, are seldom restricted to a single brain region (Bak and Chandran, 2012). The distribution of 
degenerative change is not however "diffuse, random or confluent" (Seeley et al., 2009). Rather, it follows an orderly and sequential process, affecting brain networks that mediate functionally related processes. Accordingly, in Amyotrophic Lateral Sclerosis (ALS) for example, the aspects of cognitive function that demonstrate the greatest impairment are those with the closest functional links to the motor system (Bak and Chandran, 2012). Frontal-striatal circuits, regardless of whether they mediate cognitive or motor processes, exhibit equivalent age-related declines in functional connectivity (Bo et al., 2014).

Concisely, neurodegenerative processes affect coherent systems constituted by the brain's intrinsic functional network architecture (Ahmed et al., 2016; Seeley et al., 2009). It is in this context that the relationships between motor and cognitive dysfunction in general, and between grip strength, frailty and cognitive decline in particular, are to be understood. Disintegration of neuronal networks that is expressed phenotypically as deficits in motor coordination and expressions of frailty will, by virtue of convergent intrinsic functional and structural covariance, also compromise cognitive function. Accordingly, declines in grip strength - in so much as they provide a marker of CNS deficits that affect the quality of motor coordination, also reflect the integrity of the whole brain networks that mediate cognition.

An ALE meta-analysis of twenty-eight fMRI studies revealed that in producing power grips, brain areas that correspond to the peak probability of converging activation across experiments included: L. precentral gyrus (area 4a), R. cerebellum (lobule V), L. SMA (area 6), L. middle cingulate cortex (area 6), R. supramarginal gyrus (hIP2 and IPC), L. rolandic operculum and L. inferior frontal gyrus (p. opercularis, area 44), L. cerebellum (lobule IV), L. precentral gyrus (area 44) and R. cerebellar vermis (lobule VI) (King et al., 2014). The point that the production of high levels of grip force imposes significant demands for coordination is emphasised by the observation that overlapping activation extrema for precision and power grips are obtained for the L. postcentral gyrus (area 3b), L. precentral gyrus (areas 4a, 44), the L. supplementary motor area (SMA, area 6), L. middle cingulate cortex (area 6), and R. cerebellum (lobules V, VI). Aside from the obvious conclusion that the application of grip force requires the engagement of a distributed brain network, it is notable that the brain activity that is generically present during all types of handgrip also encompasses the fronto-parietal cerebellar network (FPCN) - which is typically discussed in relation to cognition (e.g. Dosenbach et al., 2008), and the thalamus, rolandic operculum, and putamen (King et al., 2014). In light of the dependence upon multiple brain regions (and effective network connectivity), it might be supposed that individual variations in grip force should be closely associated with other markers of brain health. This indeed appears to be the case.

The presence of white matter hyperintensities (WMHs) is typically inferred from T2-weighted MRI. While these nonspecific changes in the cerebral white matter (aka leukoaraiosis) are commonly observed in the brains of clinically healthy middle-aged and older individuals, as a manifestation of chronic small vessel disease they are almost certainly not benign. Indeed, their prevalence has been linked to cognitive decline and dementia (e.g. Prins and Scheltens, 2015). When assessed in a random community sample of individuals aged 60-64 years, a negative relationship between the relative extent of WMHs and grip strength has been observed (Sachdev et al., 2009; 2005). In the Religious Orders Study and Memory and Aging Project, the sample is composed of 
individuals who receive annual clinical evaluations, and agree to have a brain autopsy performed after death. Within a sub-group of 791 persons followed up for 6.4 years (age at death 88.5 years), the rate of decline in grip strength was positively associated with markers of Alzheimer disease (AD) pathology (neuritic plaques, diffuse plaques, and neurofibrillary tangles in the frontal, temporal, parietal, and entorhinal cortex, and the hippocampus), and nigral neuronal loss (in the substantia nigra in the mid to rostral midbrain) (Buchman et al., 2013). Assessed over the course of the I-Lan Longitudinal Aging Study (ILAS) of community-dwelling adults aged 50 or older, individuals exhibiting cerebral (brainstem) microbleeds (CMBs) - one of the principal neuroimaging indices of cerebral small vessel diseases (CSVDs), present with lower grip strength than those without these small hemorrhages (Chung et al., 2016).

When evaluated over an interval of six years in a sample free of clinical dementia and stroke, drawn from more than two thousand members of the Framingham Offspring Study, larger declines in grip strength were associated with greater decreases in total cerebral brain volume (Camargo et al., 2016). In a group of approximately 700 individuals drawn from the Lothian Birth Cohort, who were assessed at ages 70 and 73, changes in grip strength were associated with the volume of normal-appearing white matter (i.e. areas of white matter not affected by white matter lesions). Specifically, those with the highest normal white matter (NAWM) volumes exhibited the smallest declines in maximum grip force over the two years interval (Aribisala et al., 2013) (Figure 13).

Insert Figure 13 About Here

Assessed at a single time-point in a sample aged above 64 years, individual grip strength correlates positively with concurrently derived DTI indices (fractional anisotropy (FA) and mean diffusivity (MD)) of structural brain connectivity (specifically white matter within the cingulum bundle) (Hirsiger et al., 2016). Resting state fMRI (rsfMRI) based estimates of functional brain connectivity generated for the same cohort, indicate that maximum grip strength is positively associated with greater connectivity from: i) a motor cortex seed to bilateral sensorimotor cortex and SMA; ii) a putamen seed region to the medial frontal cortex and precuneus; and iii) from cerebellar seeds to frontal cortex and temporal regions. Grip strength is also positively associated with the degree of connectivity between cerebellar Lobule V and lobules VIIIa and VIIIb (Seidler et al., 2015).

In line with the general expectation that age-related atrophy of the key elements of the cortical motor network will impact negatively on grip strength, MRI-derived indices of primary motor cortex volume are positively and linearly associated with grip strength in adults aged 65 and above (Koppelmans et al., 2015). Estimates of the area of the midbody of the corpus callosum - comprising fibres that connect homologous motor, somatosemsory and auditory cortices, correlate positively with grip strength when assessed in a large $(\approx 500)$ cohort of older adults (60-64 years) (Anstey et al., 2007). That the relationship between grip strength and markers of brain health of extends beyond the 
cortical motor network, is further emphasised by findings that both cerebellar grey matter volume and cerebellar white matter volumes are also reliable predictors of maximum grip strength (Koppelmans et al., 2015). Converging evidence has been presented for 456 participants drawn from the The I-Lan Longitudinal Aging Study. Compared to a subset of individuals without any manifestations of frailty, those who exhibited low grip strength (lowest quintile within gender) were characterized by smaller cerebellar grey matter volumes (Chen et al., 2015).

\section{Generalising the concept of shared neural substrates}

The key inference to be drawn on the basis of the present analysis is that changes in central neurological processes are a common factor mediating the close relationship that exists between lifespan variations in grip strength and cognition. The conclusion that there is a shared neural basis for expressions of motor and cognitive decline has similarly been prompted by observed associations between measures of gait sufficiency and cognitive function. Contemporary meta-analyses indicate that in healthy adults without a diagnosis of MCI or dementia, slower gait speed is associated with lower scores on: global tests of cognition (e.g. MMSE), tests of memory; tests of processing speed; and assessments of executive function (Demnitz et al., 2016). A meta-analysis of cohort studies indicates that older adults without overt neurological disease, who were classified as having slow gait velocity, exhibit an increased risk of developing dementia ((Kueper et al., 2017). Another recent meta-analysis further suggests that variability of stride time discriminates healthy older adults from those having a clinical diagnosis of MCI or dementia (Beauchet et al., 2014).

In recognition of these associations, the construct of Motoric Cognitive Risk (MCR) syndrome (Verghese et al., 2013) has been introduced. This defines a predementia syndrome characterised by cognitive complaints and slow gait speed. It was conceived of originally as a means of identifying individuals at risk for dementia in general, and for vascular dementia in particular. As with diminished grip strength, elevated mortality is attendant upon MCR syndrome (Ayers \& Verghese, 2016). Aside from the phenomenology of an association between declines in motor and cognitive function - as defined in epidemiological studies, the deductive power of the construct derives from the hypothesis that there are common regions in the brain that mediate behaviour in both domains. In more mechanistic terms, the premise is one of shared susceptibility to vascular factors and to other agents of neurodegeneration (e.g. Kueper et al., 2017). Although declines in gait speed can be registered more than ten years prior to the detection of cognitive impairment leading to a diagnosis of MCI (Buracchio et al., 2010), it is assumed that individuals who come to manifest both cognitive and motor deficits do so by reason of conjoint underlying pathophysiology (Hausdorff \& Buchman, 2013). For example, the relationships that exist between hypertension and subsequent declines in both gait speed and elements of cognition exhibit common mediation by the degree of cerebral microvascular damage (Hajjar et al., 2011).

Although there have been numerous investigations of potential associations between neural imaging derived indices of brain integrity/connectivity/pathology and the 
sufficiency of gait or cognition, in only a relatively few instances has the neurological basis of any common mediation been the explicit focus of interest. It has more typically been the case (as in section 8 above) that the existence of a shared neural substrate has been inferred from observations that a brain network (or region) conventionally held to support functions in one domain (e.g. a fronto-parietal network in cognition), is engaged in a contingent manner during the execution of a task defined in the other domain. As the findings generated through approaches of this nature have been summarised elsewhere (e.g. Hamacher et al., 2015, Wennberg et al., 2017; see also Tian et al., 2017), and they are not directly pertinent to the current review, they will not be covered here. Investigations of indices derived from neural imaging that are discriminating in relation to the expression of MCR syndrome are potentially of greater relevance. In this regard, Beauchet et al. (2016) noted that a designation of MCR syndrome was associated with: smaller volumes of total grey matter; total cortical grey matter; and volumes of grey matter in premotor cortex and prefrontal cortex. The region of the brain for which there were the largest differences in volume between those with and without MCR status was however the premotor cortex. Similarly, regional grey matter volume covariance patterns defined for the brain stem, precuneus, fusiform, motor, supplementary motor, and prefrontal (particularly ventrolateral prefrontal) cortex that are associated in nondemented community-dwelling older adults with individual variations in gait speed, also predict: speed of processing (Trail Making Test: part A); an expression of "executive function" (in a sub-cohort); but not episodic memory (Blumen et al., 2018). When evaluated using a linear regression model, an observed relationship between variations in prefrontal (in this instance the only RoI evaluated) grey matter volume and gait speed is attenuated markedly when a measure of information processing speed (Digit Symbol Substitution Test: DSTT) is included in the model (Rosano et al., 2012). This suggests that a high proportion of the shared inter-individual variation in prefrontal grey matter volume and gait speed is also common to the measure of information processing speed. In a closely related study, whereby a subset of cerebellar regions defined the RoIs for which grey matter volumes were calculated, an analogous pattern of shared association with both DSTT performance and gait speed was obtained (Nadkarni et al., 2013). Correspondingly it has been shown that statistical associations between cortical grey matter volume and gait speed are moderated by inclusion of a measure of verbal memory (Free and Cued Selective Reminding Test-Immediate Recall) (Ezzati et al., 2015). Furthermore, reports that relationships between speed of gait and: total WMH volume; WMHs located in the frontal corpus callosum; and in the right anterior thalamic radiation, are attenuated after adjustment for (DSTT derived) information processing speed - an outcome corroborated by formal mediation analysis (Bolandzadeh et al., 2014), indicate that the dependence of gait and some facets of cognition upon shared neural systems is not simply a phenomenon restricted to the cerebral grey matter.

Although typically considered a largely automated task, as with the application of grip force, walking requires that the CNS generate exquisitely timed patterns of muscle activation tailored to accommodate the demands imposed by complex biomechanical chains, and contingencies arising from interactions with a dynamic external environment. In walking, conditional sensory feedback must also be integrated to ensure an adequate level of prospective and reactive control. On this basis alone it can be deduced that there is overlap between the areas of the brain and connecting tracts that regulate certain 
aspects of cognition and motor coordination (Rosano \& Snitz, 2018). As they depend, at least in part, upon common neural systems, it is therefore to be anticipated that the neurodegenerative processes that are a feature of normal and pathological aging will exert corresponding effects on gait and cognitive sufficiency. The supporting evidence is now compelling. It serves also to emphasise the more general message of this review. The close and pervasive relationships that exist between age-related declines in motor function and expressions of cognitive dysfunction can be understood in terms of the convergent functional and structural mediation of cognitive and motor processes by the human brain.

\section{Conclusion}

The foregoing empirical studies lend additional support to the central argument advanced in the present review. Namely, the application of grip force is a complex coordinated behaviour that is mediated by integrated activity across distributed brain networks. Accordingly, individual variations in the capacity to generate grip force are closely associated with a broad spectrum of markers that reflect brain health. In view of the convergent functional and structural mediation of cognitive and motor processes by the brain, the origins of the close and pervasive relationships between age-related declines in maximum grip strength and expressions of cognitive dysfunction are thus readily appreciated. Terminal motor decline is closely associated with the onset of terminal cognitive decline (Wilson et al., 2012). Both are manifestations of common underlying pathophysiology. In marked contrast, the empirical evidence points emphatically to an absence of association between cognition and peripheral factors such as muscle mass, that have an independent influence upon individual differences in strength (Kilgour et al., 2014).

The overriding conclusion arising from the foregoing analyses is that maximum grip strength testing provides a discriminating measure of neurological function. The ramifications are clear. To the extent that changes in maximum grip strength can be registered over relatively short periods (e.g. Xue et al., 2015), they have the potential to serve as early markers of incipient changes in brain health. In presaging the accumulation of deficits that will ultimately impact negatively not only upon cognitive status, but also give rise to manifestations of frailty, loss of functional independence, and reduced quality of life, the monitoring of grip strength may assist in prognosis and facilitate early intervention.

\section{Implications}

In respect of various conditions of ageing, including those to which the designations sarcopenia or frailty have been applied, the implications of the current analysis extend beyond our understanding of aetiology. They also bear prominently upon the design and deployment of therapeutic interventions geared to combat these conditions. It is not unusual to read statements along the lines of "sarcopenia is the major 
cause of frailty" (Morley et al., 2014, page 6). Although the basis for such conclusions is typically evidence (e.g. statistical associations with expressions of frailty) derived using consensus classifications of "sarcopenia" that include indices of "muscle function" (maximum grip strength and/or walking speed), the associated discussion of causal relations is often restricted entirely to the molecular and cellular physiology of skeletal muscle (e.g. Morley, 2016). While in any domain of research there will be a range of emphases that emerge in the interpretation of empirical findings, there is presently a widespread and striking disconnect between the multifactorial measures that are used to assess "sarcopenia", and the rather uniform prescriptions that issue forth. The latter are in many instances predicated upon an interpretation of the "muscle function" dimension of "sarcopenia" determinations that excludes entirely the contributory role of the central nervous system.

This uniformity of emphasis is reflected in the pharmacological approaches being developed, or currently available, with the goal of treating "sarcopenia" (see Table 4 of Morley, 2016). These include: Vitamin D; protein (essential amino acids); testosterone; selective androgen receptor modulators (SARMS); growth hormone; ghrelin agonists, myostatin inhibitors; activin $11 \mathrm{R}$ antagonists; angiotensin converting enzyme inhibitor (perindopril); espindolol ( $\mathrm{B}_{1} / \mathrm{B}_{2}$ adrenergic receptor antagonist); and fast skeletal muscle troponin activators (Tirasemtiv). While the respective efficacy of these treatments is a matter for empirical enquiry, it is clear that the assumed nature of the underlying pathophysiology does not in most cases extend to the brain. Why is this of practical concern? The achievement of a useful endpoint in Phase II trials of such treatments may be defined in terms of some measure of muscle mass or muscle quality (i.e. consistent with the presumed mechanism of action of most of the pharmacological agents listed above). This may however prove to be entirely irrelevant if a consensus (e.g. AWGS, EWGSOP, FNIH) definition of "sarcopenia" is used to judge efficacy in Phase III clinical trials (see also Roman et al., 2013). As the analyses presented above make plain, tests of maximum grip strength (and indeed gait sufficiency) - the elements of the current consensus definitions of "sarcopenia" that have the greatest predictive power in relation to functional status, reflect primarily the integrity of the brain. Stated briefly, the steps that are being taken presently to address a perceived deficiency in muscle mass or muscle quality are quite distinct from those actually mandated by what are evidently deficits in neuromuscular control. 


\section{Acknowledgments}

The author thanks Atlantic Philanthropies for their generous support of his research through their funding of the NEIL (Neuroenhancement for Independent Lives) programme at Trinity College Institute of Neuroscience. Appreciation is extended to Mark Latash, Rachael Seidler and three anonymous reviewers, for constructive commentary. 


\section{References}

Aarsland, D., Brønnick, K., Larsen, J.P., Tysnes, O.B., Alves, G., Norwegian ParkWest Study Group, 2009. Cognitive impairment in incident, untreated Parkinson disease: the Norwegian ParkWest study. Neurology 72, 1121-1126. doi:10.1212/01.wnl.0000338632.00552.cb

Abellan van Kan, G., André, E., Bischoff Ferrari, H.A., Boirie, Y., Onder, G., Pahor, M., Ritz, P., Rolland, Y., Sampaio, C., Studenski, S., Visser, M., Vellas, B., 2009. Carla Task Force on Sarcopenia: propositions for clinical trials. J Nutr Health Aging 13, 700-707.

Abellan van Kan, G., Cesari, M., Gillette-Guyonnet, S., Dupuy, C., Nourhashémi, F., Schott, A.-M., Beauchet, O., Annweiler, C., Vellas, B., Rolland, Y., 2013. Sarcopenia and cognitive impairment in elderly women: results from the EPIDOS cohort. Age Ageing 42, 196-202. doi:10.1093/ageing/afs173

Abizanda, P., Navarro, J.L., García-Tomás, M.I., López-Jiménez, E., Martínez-Sánchez, E., Paterna, G., 2012. Validity and usefulness of hand-held dynamometry for measuring muscle strength in community-dwelling older persons. Arch Gerontol Geriatr 54, 21-27. doi:10.1016/j.archger.2011.02.006

Ahmed, R.M., Devenney, E.M., Irish, M., Ittner, A., Naismith, S., Ittner, L.M., Rohrer, J.D., Halliday, G.M., Eisen, A., Hodges, J.R., Kiernan, M.C., 2016. Neuronal network disintegration: common pathways linking neurodegenerative diseases. J Neurol Neurosurg Psychiatr. doi:10.1136/jnnp-2014-308350

Aichberger, M.C., Busch, M.A., Reischies, F.M., Ströhle, A., Heinz, A., Rapp, M.A., 2010. Effect of Physical Inactivity on Cognitive Performance after 2.5 Years of Follow-Up. GeroPsych: The Journal of Gerontopsychology and Geriatric Psychiatry 23, 7-15. doi:10.1024/1662-9647/a000003

Ailshire, J., Crimmins, E., 2013. Physical and Biological Indicators of Health and Functioning in U.S. Oldest Old. Annual Review of Gerontology and Geriatrics 33, 193-215. doi:10.1891/0198-8794.33.193

Alahmadi, A.A.S., Samson, R.S., Gasston, D., Pardini, M., Friston, K.J., D'Angelo, E., Toosy, A.T., Wheeler-Kingshott, C.A.M., 2016. Complex motor task associated with non-linear BOLD responses in cerebro-cortical areas and cerebellum. Brain Struct Funct 221, 2443-2458. doi:10.1007/s00429-015-1048-1

Alfaro-Acha, A.A., Snih, Al, S.S., Raji, M.A.M., Kuo, Y.-F.Y., Markides, K.S.K., Ottenbacher, K.J.K., 2006. Handgrip strength and cognitive decline in older Mexican Americans. J Gerontol A Biol Sci Med Sci 61, 859-865.

Allen, G., Buxton, R.B., Wong, E.C., Courchesne, E., 1997. Attentional activation of the cerebellum independent of motor involvement. Science 275, 1940-1943.

Allen, M.D., Owens, T.E., Fong, A.K., Richards, D.R., 2011. A functional neuroimaging analysis of the Trail Making Test-B: implications for clinical application. Behav Neurol 24, 159-171. doi:10.3233/BEN-2011-0278

Ambike, S.S., Paclet, F., Latash, M.L., Zatsiorsky, V.M., 2013. Grip-force modulation in multi-finger prehension during wrist flexion and extension. Exp Brain Res 227, 509522. doi:10.1007/s00221-013-3527-z

Amunts, K., Lenzen, M., Friederici, A.D., Schleicher, A., Morosan, P., PalomeroGallagher, N., Zilles, K., 2010. Broca's region: novel organizational principles and 
multiple receptor mapping. PLoS Biol 8. doi:10.1371/journal.pbio.1000489

Anderson, M.L., 2015. Mining the Brain for a New Taxonomy of the Mind. Philosophy Compass 10, 68-77. doi:10.1111/phc3.12155

Anderson, M.L., 2010. Neural reuse: a fundamental organizational principle of the brain. Behavioral and brain sciences 33, 245-66-discussion 266-313. doi: $10.1017 / \mathrm{S} 0140525 \mathrm{X} 10000853$

Anderson, M.L., Penner-Wilger, M., 2013. Neural reuse in the evolution and development of the brain: evidence for developmental homology? Developmental Psychobiology 55, 42-51. doi:10.1002/dev.21055

Anstey, K.J., Mack, H.A., Christensen, H., Li, S.-C., Reglade-Meslin, C., Maller, J., Kumar, R., Dear, K., Easteal, S., Sachdev, P., 2007. Corpus callosum size, reaction time speed and variability in mild cognitive disorders and in a normative sample. Neuropsychologia 45, 1911-1920. doi:10.1016/j.neuropsychologia.2006.11.020

Aribisala, B.S., Gow, A.J., Bastin, M.E., del Carmen Valdés Hernández, M., Murray, C., Royle, N.A., Muñoz Maniega, S., Starr, J.M., Deary, I.J., Wardlaw, J.M., 2013. Associations between level and change in physical function and brain volumes. PLoS ONE 8, e80386. doi:10.1371/journal.pone.0080386

Arsalidou, M., Duerden, E.G., Taylor, M.J., 2013. The centre of the brain: topographical model of motor, cognitive, affective, and somatosensory functions of the basal ganglia. Hum Brain Mapp 34, 3031-3054. doi:10.1002/hbm.22124

Aruin, A.S., Latash, M.L., 1995. Directional specificity of postural muscles in feedforward postural reactions during fast voluntary arm movements. Exp Brain Res 103, 323-332.

Asaka, T., Wang, Y., 2008. Effects of Aging on Feedforward Postural Synergies. Journal of Human Kinetics 20. doi:10.2478/v10078-008-0018-6

Atkinson, H.H., Rapp, S.R., Williamson, J.D., Lovato, J., Absher, J.R., Gass, M., Henderson, V.W., Johnson, K.C., Kostis, J.B., Sink, K.M., Mouton, C.P., Ockene, J.K., Stefanick, M.L., Lane, D.S., Espeland, M.A., 2010. The Relationship Between Cognitive Function and Physical Performance in Older Women: Results From the Women's Health Initiative Memory Study. J Gerontol A Biol Sci Med Sci 65A, 300306. doi:10.1093/gerona/glp149

Auyeung, T.W., Kwok, T., Lee, J., Leung, P.C., Leung, J., Woo, J., 2008. Functional decline in cognitive impairment--the relationship between physical and cognitive function. Neuroepidemiology 31, 167-173. doi:10.1159/000154929

Auyeung, T.W., Lee, J.S.W., Kwok, T., Woo, J., 2011. Physical frailty predicts future cognitive decline - a four-year prospective study in 2737 cognitively normal older adults. J Nutr Health Aging 15, 690-694.

Ayers, E., \& Verghese, J. (2016). Motoric cognitive risk syndrome and risk of mortality in older adults. Alzheimer's \& dementia: the journal of the Alzheimer's Association, 12(5), 556-564.

Backhouse, K.M., 1968. Functional anatomy of the hand. Physiotherapy 54, 114-117.

Bak, T.H., Chandran, S., 2012. What wires together dies together: verbs, actions and neurodegeneration in motor neuron disease. Cortex 48, 936-944. doi:10.1016/j.cortex.2011.07.008

Balice-Gordon, R.J., 1997. Age-related changes in neuromuscular innervation. Muscle Nerve Suppl 5, S83-7. 
Barry, B., Carson, R.G., 2004a. Transfer of resistance training to enhance rapid coordinated force production by older adults. Exp Brain Res 159, 225-238.

Barry, B., Carson, R.G., 2004b. The consequences of resistance training for movement control in older adults. Journals of Gerontology Series a-Biological Sciences and Medical Sciences 59, 730-754.

Barry, B.K., Warman, G.E., Carson, R.G., 2005. Age-related differences in rapid muscle activation after rate of force development training of the elbow flexors. Exp Brain Res 162, 122-132. doi:10.1007/s00221-004-2127-3

Barsalou, L.W., 2009. Simulation, situated conceptualization, and prediction. Philosophical Transactions of the Royal Society B: Biological Sciences 364, 1281. doi:10.1098/rstb.2008.0319

Barton, R.A., 2012. Embodied cognitive evolution and the cerebellum. Philos Trans R Soc Lond, B, Biol Sci 367, 2097-2107. doi:10.1098/rstb.2012.0112

Barton, R.A., Venditti, C., 2014. Rapid evolution of the cerebellum in humans and other great apes. Curr Biol 24, 2440-2444. doi:10.1016/j.cub.2014.08.056

Baumann, O., Borra, R.J., Bower, J.M., Cullen, K.E., Habas, C., Ivry, R.B., Leggio, M., Mattingley, J.B., Molinari, M., Moulton, E.A., Paulin, M.G., Pavlova, M.A., Schmahmann, J.D., Sokolov, A.A., 2015. Consensus paper: the role of the cerebellum in perceptual processes. Cerebellum 14, 197-220. doi:10.1007/s12311014-0627-7

Baumgartner, R.N., Waters, D.L., Gallagher, D., Morley, J.E., Garry, P.J., 1999. Predictors of skeletal muscle mass in elderly men and women. Mechanisms of Ageing and Development 107, 123-136. doi:10.1016/S0047-6374(98)00130-4

Beauchamp, M.S., Martin, A., 2007. Grounding object concepts in perception and action: evidence from fMRI studies of tools. Cortex 43, 461-468.

Belen'ki1̌, V.E., Gurfinkel, V.S., Pal'tsev, E.I., 1967. [Control elements of voluntary movements]. Biofizika 12, 135-141.

Bernard, J.A., Peltier, S.J., Wiggins, J.L., Jaeggi, S.M., Buschkuehl, M., Fling, B.W., Kwak, Y., Jonides, J., Monk, C.S., Seidler, R.D., 2013. Disrupted cortico-cerebellar connectivity in older adults. Neuroimage 83, 103-119.

doi:10.1016/j.neuroimage.2013.06.042

Bernard, J.A., Seidler, R.D., 2014. Moving forward: age effects on the cerebellum underlie cognitive and motor declines. Neuroscience and Biobehavioral Reviews 42, 193-207. doi:10.1016/j.neubiorev.2014.02.011

Bernstein, N.A., 1967. The co-ordination and regulation of movements. Pergamon Press Ltd.

Beauchet, O., Allali, G., Montero-Odasso, M., Sejdić, E., Fantino, B., \& Annweiler, C. (2014). Motor phenotype of decline in cognitive performance among communitydwellers without dementia: population-based study and meta-analysis. PloS one, 9(6), e99318.

Beauchet, O., Allali, G., Annweiler, C., \& Verghese, J. (2016). Association of motoric cognitive risk syndrome with brain volumes: results from the GAIT study. Journals of Gerontology Series A: Biomedical Sciences and Medical Sciences, 71(8), 10811088.

Bijlsma, A.Y., Meskers, C.G.M., Ling, C.H.Y., Narici, M., Kurrle, S.E., Cameron, I.D., Westendorp, R.G.J., Maier, A.B., 2013. Defining sarcopenia: the impact of different 
diagnostic criteria on the prevalence of sarcopenia in a large middle aged cohort. Age (Dordr) 35, 871-881. doi:10.1007/s11357-012-9384-Z

Blumen, H. M., Brown, L. L., Habeck, C., Allali, G., Ayers, E., Beauchet, O., ... \& Kumar, V. P. (2018). Gray matter volume covariance patterns associated with gait speed in older adults: a multi-cohort MRI study. Brain imaging and behavior, 1-15.

Bo, J., Lee, C.-M., Kwak, Y., Peltier, S.J., Bernard, J.A., Buschkuehl, M., Jaeggi, S.M., Wiggins, J.L., Jonides, J., Monk, C.S., Seidler, R.D., 2014. Lifespan differences in cortico-striatal resting state connectivity. Brain Connectivity 4, 166-180. doi:10.1089/brain.2013.0155

Bolandzadeh, N., Liu-Ambrose, T., Aizenstein, H., Harris, T., Launer, L., Yaffe, K., ... \& Rosano, C. (2014). Pathways linking regional hyperintensities in the brain and slower gait. Neuroimage, 99, 7-13.

Bohannon, R.W., 2008. Hand-Grip Dynamometry Predicts Future Outcomes in Aging Adults. J Geriatr Phys Ther 31, 3.

Boisgontier, M., Vuillerme, N., Iversen, M.D., 2010. Superimposed electrical stimulation decreases maximal grip force. J Sports Med Phys Fitness 50, 152-158.

Bouisset, S., Zattara, M., 1987. Biomechanical study of the programming of anticipatory postural adjustments associated with voluntary movement. J Biomech 20, 735-742.

Bowden, J.L., McNulty, P.A., 2013. The magnitude and rate of reduction in strength, dexterity and sensation in the human hand vary with ageing. Exp. Gerontol. 48, 756765. doi:10.1016/j.exger.2013.03.011

Bowie, C.R., Harvey, P.D., 2006. Administration and interpretation of the Trail Making Test. Nat Protoc 1, 2277-2281. doi:10.1038/nprot.2006.390

Boyes, N.G., Yee, P., Lanovaz, J.L., Farthing, J.P., 2017. Cross-education after highfrequency versus low-frequency volume-matched handgrip training. Muscle Nerve. doi:10.1002/mus.25637

Boyle, P.A., Buchman, A.S., Wilson, R.S., Leurgans, S.E., Bennett, D.A., 2010. Physical Frailty Is Associated with Incident Mild Cognitive Impairment in Community-Based Older Persons. Journal of the American Geriatrics Society 58, 248-255. doi:10.1111/j.1532-5415.2009.02671.x

Boyle, P.A., Buchman, A.S., Wilson, R.S., Leurgans, S.E., Bennett, D.A., 2009. Association of muscle strength with the risk of Alzheimer disease and the rate of cognitive decline in community-dwelling older persons. Archives of Neurology 66, 1339-1344. doi:10.1001/archneurol.2009.240

Brovelli, A., Lachaux, J.-P., Kahane, P., Boussaoud, D., 2005. High gamma frequency oscillatory activity dissociates attention from intention in the human premotor cortex. Neuroimage 28, 154-164. doi:10.1016/j.neuroimage.2005.05.045

Buchman, A.S., Schneider, J.A., Leurgans, S., Bennett, D.A., 2008. Physical frailty in older persons is associated with Alzheimer disease pathology. Neurology 71, 499504. doi:10.1212/01.wnl.0000324864.81179.6a

Buchman, A.S., Wilson, R.S., Boyle, P.A., Bienias, J.L., Bennett, D.A., 2007. Grip strength and the risk of incident Alzheimer's disease. Neuroepidemiology 29, 66-73. doi:10.1159/000109498

Buchman, A.S., Yu, L., Wilson, R.S., Boyle, P.A., Schneider, J.A., Bennett, D.A., 2014. Brain pathology contributes to simultaneous change in physical frailty and cognition in old age. J Gerontol A Biol Sci Med Sci 69, 1536-1544. doi:10.1093/gerona/glu117 
Buchman, A.S., Yu, L., Wilson, R.S., Schneider, J.A., Bennett, D.A., 2013. Association of brain pathology with the progression of frailty in older adults. Neurology 80, 2055-2061. doi:10.1212/WNL.0b013e318294b462

Bullock, T.H., 1965. Physiological bases of behavior, in: Moore, J.A. (Ed.), Ideas in Modern Biology. 1965., pp. 449-482.

Bunnell, S., Boyes, J.H., 1970. Bunnell's Surgery of the Hand, 5 ed. JP Lippincott, Philadelphia.

Buracchio, T., Dodge, H. H., Howieson, D., Wasserman, D., \& Kaye, J. (2010). The trajectory of gait speed preceding mild cognitive impairment. Archives of neurology, 67(8), 980-986.

Burssens, A., Schelpe, N., Vanhaecke, J., Dezillie, M., Stockmans, F., 2017. Influence of wrist position on maximum grip force in a post-operative orthosis. Prosthet Orthot Int 41, 78-84. doi:10.1177/0309364615605395

Calvin, W.H., 1994. The emergence of intelligence. Sci Am 271, 100-107. doi:10.1038/scientificamerican1094-100

Calvin, W.H., 1986. The river that flows uphill. The Sierra Club, San Francisco, California.

Calvin, W.H., 1983. A stone's throw and its launch window: timing precision and its implications for language and hominid brains. J. Theor. Biol. 104, 121-135.

Camargo, E.C., Weinstein, G., Beiser, A.S., Tan, Z.S., Decarli, C., Kelly-Hayes, M., Kase, C., Murabito, J.M., Seshadri, S., 2016. Association of Physical Function with Clinical and Subclinical Brain Disease: The Framingham Offspring Study. J Alzheimers Dis 53, 1597-1608. doi:10.3233/JAD-160229

Campbell, A.J., Buchner, D.M., 1997. Unstable disability and the fluctuations of frailty. Age Ageing 26, 315-318.

Carmeli, E., Patish, H., Coleman, R., 2003. The Aging Hand. J Gerontol A Biol Sci Med Sci 58, M146-M152. doi:10.1093/gerona/58.2.M146

Carson, R.G., 2006. Changes in muscle coordination with training. J Appl Physiol 101, $1506-1513$.

Cawthon, P.M., Fox, K.M., Gandra, S.R., Delmonico, M.J., Chiou, C.-F., Anthony, M.S., Sewall, A., Goodpaster, B., Satterfield, S., Cummings, S.R., Harris, T.B., Health, Aging and Body Composition Study, 2009. Do muscle mass, muscle density, strength, and physical function similarly influence risk of hospitalization in older adults? Journal of the American Geriatrics Society 57, 1411-1419. doi:10.1111/j.1532-5415.2009.02366.x

Cerminara, N.L., Lang, E.J., Sillitoe, R.V., Apps, R., 2015. Redefining the cerebellar cortex as an assembly of non-uniform Purkinje cell microcircuits. Nature Reviews Neuroscience 16, 79-93. doi:10.1038/nrn3886

Cesari, M., Onder, G., Zamboni, V., Manini, T., Shorr, R.I., Russo, A., Bernabei, R., Pahor, M., Landi, F., 2008. Physical function and self-rated health status as predictors of mortality: results from longitudinal analysis in the ilSIRENTE study. BMC Geriatr 8, 34. doi:10.1186/1471-2318-8-34

Chainani, V., Shaharyar, S., Dave, K., Choksi, V., Ravindranathan, S., Hanno, R., Jamal, O., Abdo, A., Abi Rafeh, N., 2016. Objective measures of the frailty syndrome (hand grip strength and gait speed) and cardiovascular mortality: A systematic review. Int. J. Cardiol. 215, 487-493. doi:10.1016/j.ijcard.2016.04.068 
Chan, E., MacPherson, S.E., Robinson, G., Turner, M., Lecce, F., Shallice, T., Cipolotti, L., 2015. Limitations of the trail making test part-B in assessing frontal executive dysfunction. J Int Neuropsychol Soc 21, 169-174. doi:10.1017/S135561771500003X

Charles, L.E., Burchfiel, C.M., Fekedulegn, D., Kashon, M.L., Ross, G.W., Sanderson, W.T., Petrovitch, H., 2006. Occupational and other risk factors for hand-grip strength: the Honolulu-Asia Aging Study. Occup Environ Med 63, 820-827. doi:10.1136/oem.2006.027813

Chen, L. K., Liu, L. K., Woo, J., Assantachai, P., Auyeung, T. W., Bahyah, K. S., ... \& Lee, J. S. (2014). Sarcopenia in Asia: consensus report of the Asian Working Group for Sarcopenia. Journal of the American Medical Directors Association, 15(2), 95101.

Chen, W.-T., Chou, K.-H., Liu, L.-K., Lee, P.-L., Lee, W.-J., Chen, L.-K., Wang, P.-N., Lin, C.-P., 2015. Reduced cerebellar gray matter is a neural signature of physical frailty. Hum Brain Mapp 36, 3666-3676. doi:10.1002/hbm.22870

Christensen, H., Korten, A.E., Mackinnon, A.J., Jorm, A.F., Henderson, A.S., Rodgers, B., 2000. Are changes in sensory disability, reaction time, and grip strength associated with changes in memory and crystallized Intelligence? A longitudinal analysis in an elderly community sample. Gerontology 46, 276-292.

Christensen, H., Mackinnon, A.J., Korten, A.E., Jorm, A.F., Henderson, A.S., Jacomb, P., Rodgers, B., 1999. An analysis of diversity in the cognitive performance of elderly community dwellers: Individual differences in change scores as a function of age. Psychol Aging 14, 365-379. doi:10.1037/0882-7974.14.3.365

Chung, C.-P., Chou, K.-H., Chen, W.-T., Liu, L.-K., Lee, W.-J., Chen, L.-K., Lin, C.-P., Wang, P.-N., 2016. Cerebral microbleeds are associated with physical frailty: a community-based study. Neurobiol Aging 44, 143-150. doi:10.1016/j.neurobiolaging.2016.04.025

Cicerone, K., Levin, H., Malec, J., Stuss, D., Whyte, J., 2006. Cognitive rehabilitation interventions for executive function: moving from bench to bedside in patients with traumatic brain injury. J Cogn Neurosci 18, 1212-1222. doi:10.1162/jocn.2006.18.7.1212

Clark, B.C., Manini, T.M., 2008. Sarcopenia =/= dynapenia. J Gerontol A Biol Sci Med Sci 63, 829-834.

Clark, B.C., Taylor, J.L., Hong, S.L., Law, T.D., Russ, D.W., 2015. Weaker Seniors Exhibit Motor Cortex Hypoexcitability and Impairments in Voluntary Activation. J Gerontol A Biol Sci Med Sci 70, 1112-1119. doi:10.1093/gerona/glv030

Clark, D.J., Fielding, R.A., 2012. Neuromuscular contributions to age-related weakness. J Gerontol A Biol Sci Med Sci 67, 41-47. doi:10.1093/gerona/glr041

Clark, D.J., Patten, C., Reid, K.F., Carabello, R.J., Phillips, E.M., Fielding, R.A., 2011. Muscle performance and physical function are associated with voluntary rate of neuromuscular activation in older adults. J Gerontol A Biol Sci Med Sci 66, 115-121. doi:10.1093/gerona/glq153

Clark, D.J., Patten, C., Reid, K.F., Carabello, R.J., Phillips, E.M., Fielding, R.A., 2010. Impaired voluntary neuromuscular activation limits muscle power in mobility-limited older adults. J Gerontol A Biol Sci Med Sci 65, 495-502. doi:10.1093/gerona/glq012

Clegg, A., Young, J., Iliffe, S., Rikkert, M.O., Rockwood, K., 2013. Frailty in elderly people. The Lancet. 
Clouston, S.A.P., Brewster, P., Kuh, D., Richards, M., Cooper, R., Hardy, R., Rubin, M.S., Hofer, S.M., 2013. The Dynamic Relationship Between Physical Function and Cognition in Longitudinal Aging Cohorts. Epidemiol Rev 35, 33-50. doi:10.1093/epirev/mxs004

Cooper, R., Kuh, D., Cooper, C., Gale, C.R., Lawlor, D.A., Matthews, F., Hardy, R., FALCon and HALCyon Study Teams, 2011. Objective measures of physical capability and subsequent health: a systematic review. Age Ageing 40, 14-23. doi:10.1093/ageing/afq117

Cooper, R., Kuh, D., Hardy, R., Mortality Review Group, FALCon and HALCyon Study Teams, 2010. Objectively measured physical capability levels and mortality: systematic review and meta-analysis. BMJ 341, c4467.

Cooper, R., Richards, M., Kuh, D., 2017. Childhood Cognitive Ability and Age-Related Changes in Physical Capability From Midlife: Findings From a British Birth Cohort Study. Psychosom Med 79, 785-791. doi:10.1097/PSY.0000000000000482

Cremen, I.A., Carson, R.G., 2017. Have standard tests of cognitive function been misappropriated in the study of cognitive enhancement? Front. Hum. Neurosci. 11, 276.

Cruz-Jentoft, A.J., Baeyens, J.P., Bauer, J.M., Boirie, Y., Cederholm, T., Landi, F., Martin, F.C., Michel, J.-P., Rolland, Y., Schneider, S.M., Topinková, E., Vandewoude, M., Zamboni, M., European Working Group on Sarcopenia in Older People, 2010. Sarcopenia: European consensus on definition and diagnosis: Report of the European Working Group on Sarcopenia in Older People., in:. Presented at the Age and ageing, pp. 412-423. doi:10.1093/ageing/afq034

D'Angelo, E., Casali, S., 2012. Seeking a unified framework for cerebellar function and dysfunction: from circuit operations to cognition. Front Neural Circuits 6, 116. doi:10.3389/fncir.2012.00116

D'Ausilio, A., Pulvermüller, F., Salmas, P., Bufalari, I., Begliomini, C., Fadiga, L., 2009. The motor somatotopy of speech perception. Curr Biol 19, 381-385. doi:10.1016/j.cub.2009.01.017

Dam, T.-T., Peters, K.W., Fragala, M., Cawthon, P.M., Harris, T.B., McLean, R., Shardell, M., Alley, D.E., Kenny, A., Ferrucci, L., Guralnik, J., Kiel, D.P., Kritchevsky, S., Vassileva, M.T., Studenski, S., 2014. An evidence-based comparison of operational criteria for the presence of sarcopenia. J Gerontol A Biol Sci Med Sci 69, 584-590. doi:10.1093/gerona/glu013

Danielmeier, C., Eichele, T., Forstmann, B.U., Tittgemeyer, M., Ullsperger, M., 2011. Posterior medial frontal cortex activity predicts post-error adaptations in task-related visual and motor areas. Journal of Neuroscience 31, 1780-1789. doi:10.1523/JNEUROSCI.4299-10.2011

Darbin, O., 2012. The aging striatal dopamine function. Parkinsonism Relat Disord 18, 426-432. doi:10.1016/j.parkreldis.2011.11.025

Darwin, C., 1871. The descent of man, 2 Vols. London 81, 130-131.

De Buyser, S.L., Petrovic, M., Taes, Y.E., Toye, K.R.C., Kaufman, J.-M., Goemaere, S., 2013. Physical function measurements predict mortality in ambulatory older men.

Eur. J. Clin. Invest. 43, 379-386. doi:10.1111/eci.12056

de Jong, B.M., van Zomeren, A.H., Willemsen, A.T., Paans, A.M., 1996. Brain activity related to serial cognitive performance resembles circuitry of higher order motor 
control. Exp Brain Res 109, 136-140.

de Jong, L.W., Wang, Y., White, L.R., Yu, B., van Buchem, M.A., Launer, L.J., 2012.

Ventral striatal volume is associated with cognitive decline in older people: a population based MR-study. Neurobiol Aging 33, 424.e1-10.

doi:10.1016/j.neurobiolaging.2010.09.027

de Lange, F.P., Rahnev, D.A., Donner, T.H., Lau, H., 2013. Prestimulus oscillatory activity over motor cortex reflects perceptual expectations. Journal of Neuroscience 33, 1400-1410. doi:10.1523/JNEUROSCI.1094-12.2013

Deary, I.J., Johnson, W., Gow, A.J., Pattie, A., Brett, C.E., Bates, T.C., Starr, J.M., 2011. Losing one's grip: a bivariate growth curve model of grip strength and nonverbal reasoning from age 79 to 87 years in the Lothian Birth Cohort 1921. J Gerontol B Psychol Sci Soc Sci 66, 699-707. doi:10.1093/geronb/gbr059

Deary, I.J., Whalley, L.J., Batty, G.D., Starr, J.M., 2006. Physical fitness and lifetime cognitive change. Neurology 67, 1195-1200. doi:10.1212/01.wnl.0000238520.06958.6a

Dehaene, S., Cohen, L., 1997. Cerebral pathways for calculation: Double dissociation between rote verbal and quantitative knowledge of arithmetic. Cortex 33, 219-250.

Dehaene, S., Cohen, L., 1995. Towards an anatomical and functional model of number processing. Mathematical cognition 1, 83-120.

Dehaene, S., Piazza, M., Pinel, P., Cohen, L., 2003. Three parietal circuits for number processing. Cogn Neuropsychol 20, 487-506. doi:10.1080/02643290244000239

Dehaene, S., Tzourio, N., Frak, V., Raynaud, L., Cohen, L., Mehler, J., Mazoyer, B., 1996. Cerebral activations during number multiplication and comparison: a PET study. Neuropsychologia 34, 1097-1106.

Delbono, O., 2003. Neural control of aging skeletal muscle. Aging cell.

Delmonico, M.J., Harris, T.B., Visser, M., Park, S.W., Conroy, M.B., Velasquez-Mieyer, P., et al. (2009). Longitudinal study of muscle strength, quality, and adipose tissue infiltration. The American Journal of Clinical Nutrition, 90(6), 1579-1585.

Demnitz, N., Esser, P., Dawes, H., Valkanova, V., Johansen-Berg, H., Ebmeier, K. P., \& Sexton, C. (2016). A systematic review and meta-analysis of cross-sectional studies examining the relationship between mobility and cognition in healthy older adults. Gait \& posture, 50, 164-174.

Deschenes, M.R., Roby, M.A., Eason, M.K., Harris, M.B., 2010. Remodeling of the neuromuscular junction precedes sarcopenia related alterations in myofibers. Exp. Gerontol. 45, 389-393. doi:10.1016/j.exger.2010.03.007

deVries, H.A., Wiswell, R.A., Romero, G.T., Heckathorne, E., 1985. Changes with age in monosynaptic reflexes elicited by mechanical and electrical stimulation. Am J Phys Med 64, 71-81.

Diermayr, G., McIsaac, T.L., Gordon, A.M., 2011. Finger force coordination underlying object manipulation in the elderly - a mini-review. Gerontology 57, 217-227. doi:10.1159/000295921

Donner, T.H., Siegel, M., Fries, P., Engel, A.K., 2009. Buildup of choice-predictive activity in human motor cortex during perceptual decision making. Curr Biol 19, 1581-1585. doi:10.1016/j.cub.2009.07.066

Donner, T.H., Siegel, M., Oostenveld, R., Fries, P., Bauer, M., Engel, A.K., 2007. Population activity in the human dorsal pathway predicts the accuracy of visual 
motion detection. J Neurophysiol 98, 345-359. doi:10.1152/jn.01141.2006

Dosenbach, N.U.F., Fair, D.A., Cohen, A.L., Schlaggar, B.L., Petersen, S.E., 2008. A dual-networks architecture of top-down control. Trends in Cognitive Sciences 12, 99-105. doi:10.1016/j.tics.2008.01.001

E, K.-H., Chen, S.-H.A., Ho, M.-H.R., Desmond, J.E., 2014. A meta-analysis of cerebellar contributions to higher cognition from PET and fMRI studies. Hum Brain Mapp 35, 593-615. doi:10.1002/hbm.22194

Eisen, A., Entezari-Taher, M., Stewart, H., 1996. Cortical projections to spinal motoneurons: changes with aging and amyotrophic lateral sclerosis. Neurology 46, 1396-1404.

El-Sais, W.M., Mohammad, W.S., 2014. INFLUENCE OF DIFFERENT TESTING POSTURES ON HAND GRIP STRENGTH. European Scientific Journal 10, 290301.

Emmelot-Vonk, M.H., Verhaar, H.J.J., Nakhai Pour, H.R., Aleman, A., Lock, T.M.T.W., Bosch, J.L.H.R., Grobbee, D.E., van der Schouw, Y.T., 2008. Effect of testosterone supplementation on functional mobility, cognition, and other parameters in older men: a randomized controlled trial. Jama 299, 39-52. doi:10.1001/jama.2007.51

Enoka, R.M., 1988. Muscle strength and its development. New perspectives. Sports Medicine 6, 146-168.

Ensrud, K.E., Nevitt, M.C., Yunis, C., Cauley, J.A., Seeley, D.G., Fox, K.M., Cummings, S.R., 1994. Correlates of impaired function in older women. Journal of the American Geriatrics Society 42, 481-489.

Ezzati, A., Katz, M. J., Lipton, M. L., Lipton, R. B., \& Verghese, J. (2015). The association of brain structure with gait velocity in older adults: a quantitative volumetric analysis of brain MRI. Neuroradiology, 57(8), 851-861.

Farthing, J.P., Krentz, J.R., Magnus, C.R.A., Barss, T.S., Lanovaz, J.L., Cummine, J., Esopenko, C., Sarty, G.E., Borowsky, R., 2011. Changes in functional magnetic resonance imaging cortical activation with cross education to an immobilized limb. Med Sci Sports Exerc 43, 1394-1405. doi:10.1249/MSS.0b013e318210783c

Felicio, D.C., Pereira, D.S., Assumpção, A.M., de Jesus-Moraleida, F.R., de Queiroz, B.Z., da Silva, J.P., de Brito Rosa, N.M., Dias, J.M.D., Pereira, L.S.M., 2014. Poor correlation between handgrip strength and isokinetic performance of knee flexor and extensor muscles in community-dwelling elderly women. Geriatr Gerontol Int 14, 185-189. doi:10.1111/ggi.12077

Ferstl, E.C., Neumann, J., Bogler, C., Cramon, von, D.Y., 2008. The extended language network: a meta-analysis of neuroimaging studies on text comprehension. Hum Brain Mapp 29, 581-593. doi:10.1002/hbm.20422

Fielding, R.A., Vellas, B., Evans, W.J., Bhasin, S., Morley, J.E., Newman, A.B., Abellan van Kan, G., Andrieu, S., Bauer, J., Breuille, D., Cederholm, T., Chandler, J., De Meynard, C., Donini, L., Harris, T., Kannt, A., Keime Guibert, F., Onder, G., Papanicolaou, D., Rolland, Y., Rooks, D., Sieber, C., Souhami, E., Verlaan, S., Zamboni, M., 2011. Sarcopenia: an undiagnosed condition in older adults. Current consensus definition: prevalence, etiology, and consequences. International working group on sarcopenia. J Am Med Dir Assoc 12, 249-256.

doi:10.1016/j.jamda.2011.01.003

Frank, M.J., Loughry, B., O'Reilly, R.C., 2001. Interactions between frontal cortex and 
basal ganglia in working memory: a computational model. Cogn Affect Behav Neurosci 1, 137-160.

Fried, L.P., Ferrucci, L., Darer, J., Williamson, J.D., Anderson, G., 2004. Untangling the concepts of disability, frailty, and comorbidity: implications for improved targeting and care. J Gerontol A Biol Sci Med Sci 59, 255-263.

Fried, L.P.L., Tangen, C.M.C., Walston, J.J., Newman, A.B.A., Hirsch, C.C., Gottdiener, J.J., Seeman, T.T., Tracy, R.R., Kop, W.J.W., Burke, G.G., McBurnie, M.A.M., 2001. Frailty in older adults: evidence for a phenotype. J Gerontol A Biol Sci Med Sci 56, M146-M156.

Gale, C.R., Martyn, C.N., Cooper, C., Sayer, A.A., 2006. Grip strength, body composition, and mortality. International Journal of Epidemiology 36, 228-235. doi:10.1093/ije/dyl224

Gallucci, M., Mazzuco, S., Ongaro, F., Di Giorgi, E., Mecocci, P., Cesari, M., Albani, D., Forloni, G.L., Durante, E., Gajo, G.B., Zanardo, A., Siculi, M., Caberlotto, L., Regini, C., 2013. Body mass index, lifestyles, physical performance and cognitive decline: the "Treviso Longeva (TRELONG)" study. J Nutr Health Aging 17, 378-384. doi:10.1007/s12603-012-0397-1

Gatz, M., Reynolds, C.A., Finkel, D., Pedersen, N.L., Walters, E., 2010. Dementia in Swedish twins: predicting incident cases. Behav. Genet. 40, 768-775. doi:10.1007/s10519-010-9407-4

Gelfand, I.M., Tsetlin, M.L., 1966. On mathematical modeling of the mechanisms of the central nervous system, in: Gelfand, I., Gurfinkel, S., Fomin, S., Tsetlin, M. (Eds.). Nauka Moscow, pp. 9-26.

Giampaoli, S., Ferrucci, L., Cecchi, F., Noce, Lo, C., Poce, A., Dima, F., Santaquilani, A., Vescio, M.F., Menotti, A., 1999. Hand-grip strength predicts incident disability in non-disabled older men. Age Ageing 28, 283-288.

Glahn, D.C., Ragland, J.D., Abramoff, A., Barrett, J., Laird, A.R., Bearden, C.E., Velligan, D.I., 2005. Beyond hypofrontality: a quantitative meta-analysis of functional neuroimaging studies of working memory in schizophrenia. Hum Brain Mapp 25, 60-69. doi:10.1002/hbm.20138

Gluth, S., Rieskamp, J., Büchel, C., 2013. Classic EEG motor potentials track the emergence of value-based decisions. Neuroimage 79, 394-403. doi:10.1016/j.neuroimage.2013.05.005

Gluth, S., Rieskamp, J., Büchel, C., 2012. Deciding when to decide: time-variant sequential sampling models explain the emergence of value-based decisions in the human brain. Journal of Neuroscience 32, 10686-10698. doi:10.1523/JNEUROSCI.0727-12.2012

Goldberger, M.E., 1969. The extrapyramidal systems of the spinal cord. II. Results of combined pyramidal and extrapyramidal lesions in the macaque. J Comp Neurol 135, 1-26. doi:10.1002/cne.901350102

Goodpaster, B.H., Park, S.W., Harris, T.B., Kritchevsky, S.B., Nevitt, M., Schwartz, A.V., Simonsick, E.M., Tylavsky, F.A., Visser, M., Newman, A.B., 2006. The loss of skeletal muscle strength, mass, and quality in older adults: the health, aging and body composition study. J Gerontol A Biol Sci Med Sci 61, 1059-1064.

Gordon, A.M., Huxley, A.F., Julian, F.J., 1966. The variation in isometric tension with sarcomere length in vertebrate muscle fibres. The Journal of Physiology 184, 170- 
192.

Gorniak, S.L., Zatsiorsky, V.M., Latash, M.L., 2011. Manipulation of a fragile object by elderly individuals. Exp Brain Res 212, 505-516. doi:10.1007/s00221-011-2755-3

Graff-Radford, J., Williams, L., Jones, D. T., \& Benarroch, E. E. (2017). Caudate nucleus as a component of networks controlling behavior. Neurology, 89(21), 2192-2197.

Graybiel, A.M., 1997. The basal ganglia and cognitive pattern generators. Schizophr Bull 23, 459-469.

Grent-'t-Jong, T., Oostenveld, R., Jensen, O., Medendorp, W.P., Praamstra, P., 2013. Oscillatory dynamics of response competition in human sensorimotor cortex. Neuroimage 83, 27-34. doi:10.1016/j.neuroimage.2013.06.051

Hagen, K., Ehlis, A.-C., Haeussinger, F.B., Heinzel, S., Dresler, T., Mueller, L.D., Herrmann, M.J., Fallgatter, A.J., Metzger, F.G., 2014. Activation during the Trail Making Test measured with functional near-infrared spectroscopy in healthy elderly subjects. Neuroimage 85 Pt 1, 583-591. doi:10.1016/j.neuroimage.2013.09.014

Hajjar, I., Quach, L., Yang, F., Chaves, P. H., Newman, A. B., Mukamal, K., ... \& Lipsitz, L. A. (2011). Hypertension, white matter hyperintensities, and concurrent impairments in mobility, cognition, and mood: the Cardiovascular Health Study. Circulation, 123, 858-865.

Hairi, N.N., Cumming, R.G., Naganathan, V., Handelsman, D.J., Le Couteur, D.G., Creasey, H., Waite, L.M., Seibel, M.J., Sambrook, P.N., 2010. Loss of Muscle Strength, Mass (Sarcopenia), and Quality (Specific Force) and Its Relationship with Functional Limitation and Physical Disability: The Concord Health and Ageing in Men Project. Journal of the American Geriatrics Society 58, 2055-2062. doi:10.1111/j.1532-5415.2010.03145.x

Halla B Olafsdottir, S.W.K.V.M.Z.M.L.L., 2008. Anticipatory synergy adjustments in preparation to self-triggered perturbations in elderly individuals. J Appl Biomech 24, 175.

Hamer, M., Stamatakis, E., 2013. Screen-based sedentary behavior, physical activity, and muscle strength in the English longitudinal study of ageing. PLoS ONE 8, e66222. doi:10.1371/journal.pone.0066222

Hamacher, D., Herold, F., Wiegel, P., Hamacher, D., \& Schega, L. (2015). Brain activity during walking: a systematic review. Neuroscience \& Biobehavioral Reviews, 57, 310-327.

Hanakawa, T., Honda, M., Sawamoto, N., Okada, T., Yonekura, Y., Fukuyama, H., Shibasaki, H., 2002. The role of rostral Brodmann area 6 in mental-operation tasks: an integrative neuroimaging approach. Cereb Cortex 12, 1157-1170.

Harridge, S.D., Kryger, A., Stensgaard, A., 1999. Knee extensor strength, activation, and size in very elderly people following strength training. Muscle Nerve 22, 831-839.

Hausdorff, J. M., \& Buchman, A. S. (2013). What links gait speed and MCI with dementia? A fresh look at the association between motor and cognitive function. The Journals of Gerontology Series a, Biological Sciences and Medical Sciences, 68(4), 409-411.

Hedden, T., Schultz, A.P., Rieckmann, A., Mormino, E.C., Johnson, K.A., Sperling, R.A., Buckner, R.L., 2016. Multiple Brain Markers are Linked to Age-Related Variation in Cognition. CEREBRAL CORTEX 26, 1388-1400. doi:10.1093/cercor/bhu238

Heekeren, H.R., Marrett, S., Ruff, D.A., Bandettini, P.A., Ungerleider, L.G., 2006. 
Involvement of human left dorsolateral prefrontal cortex in perceptual decision making is independent of response modality. Proceedings of the National Academy of Sciences of the United States of America 103, 10023-10028. doi:10.1073/pnas.0603949103

Hélie, S., Ell, S.W., Ashby, F.G., 2015. Learning robust cortico-cortical associations with the basal ganglia: an integrative review. Cortex 64, 123-135. doi:10.1016/j.cortex.2014.10.011

Hepple, R. T. (2012). Muscle atrophy is not always sarcopenia. Journal of Applied Physiology, 113(4), 677-679.

Hirsiger, S., Koppelmans, V., Mérillat, S., Liem, F., Erdeniz, B., Seidler, R.D., Jäncke, L., 2016. Structural and functional connectivity in healthy aging: Associations for cognition and motor behavior. Hum Brain Mapp 37, 855-867. doi:10.1002/hbm.23067

Hirose, D., Hanyu, H., Fukasawa, R., Namioka, N., Hatanaka, H., \& Sato, T. (2017). Diabetes - related dementia is associated with dynapenia, but not with sarcopenia. Geriatrics \& gerontology international, 17(1), 175-177.

Hodkinson, H.M., 1972. Evaluation of a mental test score for assessment of mental impairment in the elderly. Age Ageing.

Hoffstaedter, F., Grefkes, C., Caspers, S., Roski, C., Palomero-Gallagher, N., Laird, A.R., Fox, P.T., Eickhoff, S.B., 2014. The role of anterior midcingulate cortex in cognitive motor control: evidence from functional connectivity analyses. Hum Brain Mapp 35, 2741-2753. doi:10.1002/hbm.22363

Hogan, M.J., Staff, R.T., Bunting, B.P., Murray, A.D., Ahearn, T.S., Deary, I.J., Whalley, L.J., 2011. Cerebellar brain volume accounts for variance in cognitive performance in older adults. Cortex 47, 441-450. doi:10.1016/j.cortex.2010.01.001

Hopkins, W.D., Russell, J.L., Schaeffer, J.A., 2012. The neural and cognitive correlates of aimed throwing in chimpanzees: a magnetic resonance image and behavioural study on a unique form of social tool use. Philos Trans R Soc Lond, B, Biol Sci 367, 37-47. doi:10.1098/rstb.2011.0195

Houk, J.C., Bastianen, C., Fansler, D., Fishbach, A., Fraser, D., Reber, P.J., Roy, S.A., Simo, L.S., 2007. Action selection and refinement in subcortical loops through basal ganglia and cerebellum. Philos Trans R Soc Lond, B, Biol Sci 362, 1573-1583. doi:10.1098/rstb.2007.2063

Huang, C. Y., Hwang, A. C., Liu, L. K., Lee, W. J., Chen, L. Y., Peng, L. N., ... \& Chen, L. K. (2016). Association of dynapenia, sarcopenia, and cognitive impairment among community-dwelling older Taiwanese. Rejuvenation research, 19(1), 71-78.

Hubbard, R.E., O'Mahony, M.S., Woodhouse, K.W., 2009. Characterising frailty in the clinical setting--a comparison of different approaches. Age Ageing 38, 115-119. doi:10.1093/ageing/afn252

Hyatt, R.H., Whitelaw, M.N., Bhat, A., Scott, S., MAXWELL, J.D., 1990. Association of muscle strength with functional status of elderly people.

Imamizu, H., Kawato, M., 2009. Brain mechanisms for predictive control by switching internal models: implications for higher-order cognitive functions. Psychol Res 73, 527-544. doi:10.1007/s00426-009-0235-1

Incel, N.A., Sezgin, M., As, I., Cimen, O.B., Sahin, G., 2009. The geriatric hand: correlation of hand-muscle function and activity restriction in elderly. Int J Rehabil 
Res 32, 213-218. doi:10.1097/MRR.0b013e3283298226

Inglin, B., Woollacott, M., 1988. Age-related changes in anticipatory postural adjustments associated with arm movements. J Gerontol 43, M105-13.

Ito, M., 2008. Control of mental activities by internal models in the cerebellum. Nature Reviews Neuroscience 9, 304-313. doi:10.1038/nrn2332

Ito, M., 1993. New concepts in cerebellar function. Rev. Neurol. (Paris) 149, 596-599.

Ivry, R., 1997. Cerebellar timing systems. Int. Rev. Neurobiol. 41, 555-573.

Incel, N.A., 2014. Hand Function in Geriatric Conditions, in: Duruoz, M.T. (Ed.), Hand Function. Hand Function, New York, pp. 171-183.

Iwamura, M., \& Kanauchi, M. (2017). A cross-sectional study of the association between dynapenia and higher-level functional capacity in daily living in community-dwelling older adults in Japan. BMC geriatrics, 17(1), 1.

Jacobson, S.C., Blanchard, M., Connolly, C.C., Cannon, M., Garavan, H., 2011. An fMRI investigation of a novel analogue to the Trail-Making Test. Brain and Cognition 77, 60-70. doi:10.1016/j.bandc.2011.06.001

Jaeggi, S.M., Seewer, R., Nirkko, A.C., Eckstein, D., Schroth, G., Groner, R., Gutbrod, K., 2003. Does excessive memory load attenuate activation in the prefrontal cortex? Load-dependent processing in single and dual tasks: functional magnetic resonance imaging study. Neuroimage 19, 210-225.

Jang, Y.C., Van Remmen, H., 2011. Age-associated alterations of the neuromuscular junction. Exp. Gerontol. 46, 193-198. doi:10.1016/j.exger.2010.08.029

Jenkins, N.D.M., Buckner, S.L., Bergstrom, H.C., Cochrane, K.C., Goldsmith, J.A., Housh, T.J., Johnson, G.O., Schmidt, R.J., Cramer, J.T., 2014. Reliability and relationships among handgrip strength, leg extensor strength and power, and balance in older men. Exp. Gerontol. 58C, 47-50. doi:10.1016/j.exger.2014.07.007

Jirak, D., Menz, M.M., Buccino, G., Borghi, A.M., Binkofski, F., 2010. Grasping language--a short story on embodiment. CONSCIOUSNESS AND COGNITION 19, 711-720. doi:10.1016/j.concog.2010.06.020

Johanson, M.E., James, M.A., Skinner, S.R., 1998. Forearm muscle activation during power grip and release. J Hand Surg Am 23, 938-944. doi:10.1016/S03635023(98)80177-9

Johnston, J.A., Bobich, L.R., Santello, M., 2010. Coordination of intrinsic and extrinsic hand muscle activity as a function of wrist joint angle during two-digit grasping. Neurosci Lett 474, 104-108. doi:10.1016/j.neulet.2010.03.017

Kallman, D.A., Plato, C.C., Tobin, J.D., 1990. The role of muscle loss in the age-related decline of grip strength: cross-sectional and longitudinal perspectives. J Gerontol 45, M82-8.

Kane, M.J., Conway, A.R.A., Miura, T.K., Colflesh, G.J.H., 2007. Working memory, attention control, and the N-back task: a question of construct validity. J Exp Psychol Learn Mem Cogn 33, 615-622. doi:10.1037/0278-7393.33.3.615

Kapur, S., Zatsiorsky, V.M., Latash, M.L., 2010. Age-related changes in the control of finger force vectors. Journal of Applied Physiology 109, 1827-1841.

doi:10.1152/japplphysiol.00430.2010

KATZ, S., FORD, A.B., MOSKOWITZ, R.W., JACKSON, B.A., JAFFE, M.W., 1963. STUDIES OF ILLNESS IN THE AGED. THE INDEX OF ADL: A STANDARDIZED MEASURE OF BIOLOGICAL AND PSYCHOSOCIAL 
FUNCTION. Jama 185, 914-919.

Keuken, M.C., Müller-Axt, C., Langner, R., Eickhoff, S.B., Forstmann, B.U., Neumann, J., 2014. Brain networks of perceptual decision-making: an fMRI ALE meta-analysis. Front. Hum. Neurosci. 8, 445. doi:10.3389/fnhum.2014.00445

Kilgour, A.H.M., Todd, O.M., Starr, J.M., 2014. A systematic review of the evidence that brain structure is related to muscle structure and their relationship to brain and muscle function in humans over the lifecourse. BMC Geriatr 14, 85. doi:10.1186/1471-2318-14-85

Kim, H., 2013. Differential neural activity in the recognition of old versus new events: an activation likelihood estimation meta-analysis. Hum Brain Mapp 34, 814-836. doi:10.1002/hbm.21474

King, M., Rauch, H.G., Stein, D.J., Brooks, S.J., 2014. The handyman's brain: a neuroimaging meta-analysis describing the similarities and differences between grip type and pattern in humans. Neuroimage $102 \mathrm{Pt}$ 2, 923-937. doi:10.1016/j.neuroimage.2014.05.064

Kinoshita, H., Kawai, S., Ikuta, K., 1995. Contributions and co-ordination of individual fingers in multiple finger prehension. Ergonomics 38, 1212-1230. doi:10.1080/00140139508925183

Kirchner, W.K., 1958. Age differences in short-term retention of rapidly changing information. Journal of Experimental Psychology 55, 352-358.

Kirkcaldie, M.T.K., Kitchener, P.D., 2007. When brains expand: mind and the evolution of cortex. Acta Neuropsychiatr 19, 139-148. doi:10.1111/j.1601-5215.2007.00204.x

Klass, M., Baudry, S., Duchateau, J., 2007. Voluntary activation during maximal contraction with advancing age: a brief review. European Journal of Applied Physiology 100, 543-551. doi:10.1007/s00421-006-0205-x

Klass, M., Baudry, S., Duchateau, J., 2005. Aging does not affect voluntary activation of the ankle dorsiflexors during isometric, concentric, and eccentric contractions. J Appl Physiol 99, 31-38. doi:10.1152/japplphysiol.01426.2004

Klein, E., Suchan, J., Moeller, K., Karnath, H.-O., Knops, A., Wood, G., Nuerk, H.-C., Willmes, K., 2014. Considering structural connectivity in the triple code model of numerical cognition: differential connectivity for magnitude processing and arithmetic facts. Brain Struct Funct. doi:10.1007/s00429-014-0951-1

Klein, P.-A., Petitjean, C., Olivier, E., Duque, J., 2014. Top-down suppression of incompatible motor activations during response selection under conflict. Neuroimage 86, 138-149. doi:10.1016/j.neuroimage.2013.08.005

Koppelmans, V., Hirsiger, S., Mérillat, S., Jäncke, L., Seidler, R.D., 2015. Cerebellar gray and white matter volume and their relation with age and manual motor performance in healthy older adults. Hum Brain Mapp 36, 2352-2363. doi:10.1002/hbm. 22775

Koziol, L.F., Budding, D.E., 2009. Subcortical Structures and Cognition. Springer Science \& Business Media.

Kueper, J. K., Speechley, M., Lingum, N. R., \& Montero-Odasso, M. (2017). Motor function and incident dementia: a systematic review and meta-analysis. Age and ageing, 46(5), 729-738.

Kuh, D., Cooper, R., Hardy, R., Guralnik, J., Richards, M., Musculoskeletal Study Team, 2009. Lifetime cognitive performance is associated with midlife physical 
performance in a prospective national birth cohort study. Psychosom Med 71, 38-48. doi:10.1097/PSY.0b013e31818a1620

Kwan, P., 2013. Sarcopenia: the gliogenic perspective. Mechanisms of Ageing and Development 134, 349-355. doi:10.1016/j.mad.2013.06.001

Laessoe, U., Voigt, M., 2008. Anticipatory postural control strategies related to predictive perturbations. Gait Posture 28, 62-68. doi:10.1016/j.gaitpost.2007.10.001

Lamm, C., Windischberger, C., Moser, E., Bauer, H., 2007. The functional role of dorsolateral premotor cortex during mental rotation: an event-related fMRI study separating cognitive processing steps using a novel task paradigm. Neuroimage 36, 1374-1386. doi:10.1016/j.neuroimage.2007.04.012

LaStayo, P., Hartzel, J., 1999. Dynamic versus static grip strength: how grip strength changes when the wrist is moved, and why dynamic grip strength may be a more functional measurement. J Hand Ther 12, 212-218.

Laukkanen, P., Heikkinen, E., Kauppinen, M., 1995. Muscle strength and mobility as predictors of survival in 75-84-year-old people. Age Ageing 24, 468-473.

Lawrence, A.D., Hodges, J.R., Rosser, A.E., Kershaw, A., ffrench-Constant, C., Rubinsztein, D.C., Robbins, T.W., Sahakian, B.J., 1998. Evidence for specific cognitive deficits in preclinical Huntington's disease. Brain 121 ( Pt 7), 1329-1341.

Lawrence, A.D., Sahakian, B.J., Hodges, J.R., Rosser, A.E., Lange, K.W., Robbins, T.W., 1996. Executive and mnemonic functions in early Huntington's disease. Brain 119 ( Pt 5), 1633-1645.

Lawton, M.P., Brody, E.M., 1969. Instrumental activities of daily living scale (IADL). Gerontologist 9, 179-186.

Lee, J.-Y., Lyoo, I.K., Kim, S.-U., Jang, H.-S., Lee, D.-W., Jeon, H.-J., Park, S.-C., Cho, M.J., 2005. Intellect declines in healthy elderly subjects and cerebellum. Psychiatry Clin. Neurosci. 59, 45-51. doi:10.1111/j.1440-1819.2005.01330.x

Lee, W.-J., Peng, L.-N., Chiou, S.-T., Chen, L.-K., 2016. Relative Handgrip Strength Is a Simple Indicator of Cardiometabolic Risk among Middle-Aged and Older People: A Nationwide Population-Based Study in Taiwan. PLoS ONE 11, e0160876. doi:10.1371/journal.pone.0160876

Legrand, D., Vaes, B., Matheï, C., Adriaensen, W., Van Pottelbergh, G., Degryse, J.M., 2014. Muscle strength and physical performance as predictors of mortality, hospitalization, and disability in the oldest old. Journal of the American Geriatrics Society 62, 1030-1038. doi:10.1111/jgs. 12840

Legrand, D., Vaes, B., Matheï, C., Swine, C., Degryse, J.M., 2013. The prevalence of sarcopenia in very old individuals according to the European consensus definition: insights from the BELFRAIL study. Age Ageing 42, 727-734. doi:10.1093/ageing/aft128

Leisman, G., Melillo, R., 2013. The basal ganglia: motor and cognitive relationships in a clinical neurobehavioral context. Reviews in the Neurosciences 24, 9-25. doi:10.1515/revneuro-2012-0067

Leong, D.P., Teo, K.K., Rangarajan, S., Lopez-Jaramillo, P., Avezum, A., Orlandini, A., Seron, P., Ahmed, S.H., Rosengren, A., Kelishadi, R., Rahman, O., Swaminathan, S., Iqbal, R., Gupta, R., Lear, S.A., Oguz, A., Yusoff, K., Zatonska, K., Chifamba, J., Igumbor, E., Mohan, V., Anjana, R.M., Gu, H., Li, W., Yusuf, S., Prospective Urban Rural Epidemiology (PURE) Study investigators, 2015. Prognostic value of grip 
strength: findings from the Prospective Urban Rural Epidemiology (PURE) study. Lancet 386, 266-273. doi:10.1016/S0140-6736(14)62000-6

Lewis, P.A., Miall, R.C., 2003. Distinct systems for automatic and cognitively controlled time measurement: evidence from neuroimaging. Curr Opin Neurobiol 13, 250-255.

Li, S., Latash, M.L., Yue, G.H., Siemionow, V., Sahgal, V., 2003. The effects of stroke and age on finger interaction in multi-finger force production tasks. Clinical Neurophysiology 114, 1646-1655.

Li, Z.-M., 2002. The influence of wrist position on individual finger forces during forceful grip. J Hand Surg Am 27, 886-896. doi:10.1053/jhsu.2002.35078

Li, Z.M., Latash, M.L., Newell, K.M., Zatsiorsky, V.M., 1998a. Motor redundancy during maximal voluntary contraction in four-finger tasks. Exp Brain Res 122, 71-78.

Li, Z.M., Latash, M.L., Zatsiorsky, V.M., 1998b. Force sharing among fingers as a model of the redundancy problem. Exp Brain Res 119, 276-286.

Liddel, E., Sherrington, C., 1925. Recruitment and some other factors of reflex inhibition. Proceedings of the Royal Society of London Series B-Biological Sciences 97, 488518.

Ling, C.H.Y., Taekema, D., de Craen, A.J.M., Gussekloo, J., Westendorp, R.G.J., Maier, A.B., 2010. Handgrip strength and mortality in the oldest old population: the Leiden 85-plus study. CMAJ 182, 429-435. doi:10.1503/cmaj.091278

Liu, X., Wang, H., Corbly, C.R., Zhang, J., Joseph, J.E., 2006. The involvement of the inferior parietal cortex in the numerical Stroop effect and the distance effect in a twodigit number comparison task. J Cogn Neurosci 18, 1518-1530.

doi:10.1162/jocn.2006.18.9.1518

Lock, M., 2013. The Alzheimer Conundrum. Princeton University Press.

Long, C., Conrad, P.W., Hall, E.A., Furler, S.L., 1970. Intrinsic-extrinsic muscle control of the hand in power grip and precision handling. An electromyographic study. $J$ Bone Joint Surg Am 52, 853-867.

MacDonald, S.W.S., DeCarlo, C.A., Dixon, R.A., 2011. Linking biological and cognitive aging: toward improving characterizations of developmental time. J Gerontol B Psychol Sci Soc Sci 66 Suppl 1, i59-70. doi:10.1093/geronb/gbr039

MacDonald, S.W.S., Dixon, R.A., Cohen, A.-L., Hazlitt, J.E., 2004. Biological age and 12-year cognitive change in older adults: findings from the Victoria Longitudinal Study. Gerontology 50, 64-81. doi:10.1159/000075557

MacLullich, A.M.J., Edmond, C.L., Ferguson, K.J., Wardlaw, J.M., Starr, J.M., Seckl, J.R., Deary, I.J., 2004. Size of the neocerebellar vermis is associated with cognition in healthy elderly men. Brain and Cognition 56, 344-348.

doi:10.1016/j.bandc.2004.08.001

Malmstrom, T.K., Wolinsky, F.D., Andresen, E.M., Miller, J.P., Miller, D.K., 2005. Cognitive ability and physical performance in middle-aged African Americans. Journal of the American Geriatrics Society 53, 997-1001. doi:10.1111/j.15325415.2005.53318.x

Man'kovskii, N.B., Mints AYa, Lysenyuk, V.P., 1980. Regulation of the preparatory period for complex voluntary movement in old and extreme old age. Human Physiology 6, 46-50.

Manini, T. M., \& Clark, B. C. (2012). Dynapenia and aging: an update. Journals of Gerontology Series A: Biomedical Sciences and Medical Sciences, 67(1), 28-40. 
Manini, T.M., Hong, S.L., Clark, B.C., 2013. Aging and muscle: a neuron's perspective. Curr Opin Clin Nutr Metab Care 16, 21-26. doi:10.1097/MCO.0b013e32835b5880

Marmon, A.R., Pascoe, M.A., Schwartz, R.S., Enoka, R.M., 2011. Associations among strength, steadiness, and hand function across the adult life span. Med Sci Sports Exerc 43, 560-567. doi:10.1249/MSS.0b013e3181f3f3ab

Martin, A., 2007. The representation of object concepts in the brain. Annu. Rev. Psychol. 58, 25-45. doi:10.1146/annurev.psych.57.102904.190143

Martin, J.A., Ramsay, J., Hughes, C., Peters, D.M., Edwards, M.G., 2015. Age and grip strength predict hand dexterity in adults. PLoS ONE 10, e0117598. doi:10.1371/journal.pone.0117598

Massion, J., 1992. Movement, posture and equilibrium: interaction and coordination. Prog Neurobiol 38, 35-56.

McGough, E.L., Cochrane, B.B., Pike, K.C., Logsdon, R.G., McCurry, S.M., Teri, L., 2013. Dimensions of physical frailty and cognitive function in older adults with amnestic mild cognitive impairment. Ann Phys Rehabil Med 56, 329-341. doi:10.1016/j.rehab.2013.02.005

McHaffie, J.G., Stanford, T.R., Stein, B.E., Coizet, V., Redgrave, P., 2005. Subcortical loops through the basal ganglia. Trends Neurosci 28, 401-407. doi:10.1016/j.tins.2005.06.006

McLean, R.R., Shardell, M.D., Alley, D.E., Cawthon, P.M., Fragala, M.S., Harris, T.B., Kenny, A.M., Peters, K.W., Ferrucci, L., Guralnik, J.M., Kritchevsky, S.B., Kiel, D.P., Vassileva, M.T., Xue, Q.-L., Perera, S., Studenski, S.A., Dam, T.-T.L., 2014. Criteria for clinically relevant weakness and low lean mass and their longitudinal association with incident mobility impairment and mortality: the foundation for the National Institutes of Health (FNIH) sarcopenia project. J Gerontol A Biol Sci Med Sci 69, 576-583. doi:10.1093/gerona/glu012

Meincke, R.H., Osler, M., Mortensen, E.L., Hansen, Å.M., 2016. Is Intelligence in Early Adulthood Associated With Midlife Physical Performance Among Danish Males? J Aging Health 28, 530-545. doi:10.1177/0898264315594139

Mendoza, G., Merchant, H., 2014. Motor system evolution and the emergence of high cognitive functions. Prog Neurobiol 122, 73-93.

doi:10.1016/j.pneurobio.2014.09.001

Metter, E.J., Talbot, L.A., Schrager, M., Conwit, R., 2002. Skeletal muscle strength as a predictor of all-cause mortality in healthy men. J Gerontol A Biol Sci Med Sci 57, B359-65.

Michelet, T., Duncan, G.H., Cisek, P., 2010. Response competition in the primary motor cortex: corticospinal excitability reflects response replacement during simple decisions. J Neurophysiol 104, 119-127. doi:10.1152/jn.00819.2009

Middleton, F.A., 2003. Fundamental and clinical evidence for basal ganglia influences on cognition, in: Bédard, M.-A. (Ed.). Mental and behavioral dysfunction in movement disorders. Humana Press, pp. 13-33.

Middleton, F.A., Strick, P.L., 2000a. Basal ganglia output and cognition: evidence from anatomical, behavioral, and clinical studies. Brain and Cognition 42, 183-200. doi:10.1006/brcg.1999.1099

Middleton, F.A., Strick, P.L., 2000b. Basal ganglia and cerebellar loops: motor and cognitive circuits. Brain Res Brain Res Rev 31, 236-250. 
Miller, E.K., 2013. The "working” of working memory. Dialogues Clin Neurosci 15, 411-418.

Miller, T.D., Ferguson, K.J., Reid, L.M., Wardlaw, J.M., Starr, J.M., Seckl, J.R., Deary, I.J., MacLullich, A.M.J., 2013. Cerebellar vermis size and cognitive ability in community-dwelling elderly men. Cerebellum 12, 68-73. doi:10.1007/s12311-0120397-z

Mitnitski, A.B., Mogilner, A.J., Rockwood, K., 2001. Accumulation of deficits as a proxy measure of aging. ScientificWorldJournal 1, 323-336. doi:10.1100/tsw.2001.58

Moeller, K., Willmes, K., Klein, E., 2015. A review on functional and structural brain connectivity in numerical cognition. Front. Hum. Neurosci. 9, 227. doi:10.3389/fnhum.2015.00227

Mogk, J.P.M., Keir, P.J., 2003. The effects of posture on forearm muscle loading during gripping. Ergonomics 46, 956-975. doi:10.1080/0014013031000107595

Moll, J., de Oliveira-Souza, R., Moll, F.T., Bramati, I.E., Andreiuolo, P.A., 2002. The cerebral correlates of set-shifting: an fMRI study of the trail making test. Arq Neuropsiquiatr 60, 900-905.

Morgan, M., Phillips, J.G., Bradshaw, J.L., Mattingley, J.B., Iansek, R., Bradshaw, J.A., 1994. Age-related motor slowness: simply strategic? J Gerontol 49, M133-9.

Morley, J.E., Abbatecola, A.M., Argiles, J.M., Baracos, V., Bauer, J., Bhasin, S., Cederholm, T., Coats, A.J.S., Cummings, S.R., Evans, W.J., Fearon, K., Ferrucci, L., Fielding, R.A., Guralnik, J.M., Harris, T.B., Inui, A., Kalantar-Zadeh, K., Kirwan, B.-A., Mantovani, G., Muscaritoli, M., Newman, A.B., Rossi-Fanelli, F., Rosano, G.M.C., Roubenoff, R., Schambelan, M., Sokol, G.H., Storer, T.W., Vellas, B., Haehling, von, S., Yeh, S.-S., Anker, S.D., Society on Sarcopenia, Cachexia and Wasting Disorders Trialist Workshop, 2011. Sarcopenia with limited mobility: an international consensus. J Am Med Dir Assoc 12, 403-409. doi:10.1016/j.jamda.2011.04.014

Morley, J. E. (2016). Pharmacologic options for the treatment of sarcopenia. Calcified tissue international, 98(4), 319-333.

Morley, J. E., von Haehling, S., Anker, S. D., \& Vellas, B. (2014). From sarcopenia to frailty: a road less traveled. Journal of cachexia, sarcopenia and muscle, 5(1), 5-8.

Morse, C.I., Thom, J.M., Davis, M.G., Fox, K.R., Birch, K.M., Narici, M.V., 2004. Reduced plantarflexor specific torque in the elderly is associated with a lower activation capacity. European Journal of Applied Physiology 92, 219-226. doi:10.1007/s00421-004-1056-y

Mourao-Miranda, J., Ecker, C., Sato, J.R., Brammer, M., 2009. Dynamic changes in the mental rotation network revealed by pattern recognition analysis of fMRI data. J Cogn Neurosci 21, 890-904. doi:10.1162/jocn.2009.21078

Müller, L.D., Guhn, A., Zeller, J.B.M., Biehl, S.C., Dresler, T., Hahn, T., Fallgatter, A.J., Polak, T., Deckert, J., Herrmann, M.J., 2014. Neural correlates of a standardized version of the trail making test in young and elderly adults: a functional near-infrared spectroscopy study. Neuropsychologia 56, 271-279.

doi:10.1016/j.neuropsychologia.2014.01.019

Nadkarni, N. K., Nunley, K. A., Aizenstein, H., Harris, T. B., Yaffe, K., Satterfield, S., ... \& Health ABC Study. (2013). Association between cerebellar gray matter volumes, gait speed, and information-processing ability in older adults enrolled in the Health 
ABC study. Journals of Gerontology Series A: Biomedical Sciences and Medical Sciences, 69(8), 996-1003.

Nam, Y.-S., Lee, G., Yun, J.M., Cho, B., 2018. Testosterone Replacement, Muscle Strength, and Physical Function. World J Mens Health. doi:10.5534/wjmh.182001

Napier, J.R., 1966. Functional aspects of the anatomy of the hand, in: Pulvertaft, R.G. (Ed.). Clinical Surgery. Butterworths, London.

Narayana, S., Laird, A.R., Tandon, N., Franklin, C., Lancaster, J.L., Fox, P.T., 2012. Electrophysiological and functional connectivity of the human supplementary motor area. Neuroimage 62, 250-265. doi:10.1016/j.neuroimage.2012.04.060

Neubert, F.X., Mars, R.B., Rushworth, M.F., 2013. Is there an inferior frontal cortical network for cognitive control and inhibition. Principles of frontal lobe ....

Neves, T., Lopes, M. B. M., Souza, M. C., Ferriolli, E., Fett, C. A., \& Fett, W. R. (2018). Sarcopenia versus dynapenia" functional performance and physical disability in cross sectional study. Journal of Aging Research and Clinical Practice, 7, 60-68.

Newman, A.B., Kupelian, V., Visser, M., Simonsick, E.M., Goodpaster, B.H., Kritchevsky, S.B., Tylavsky, F.A., Rubin, S.M., Harris, T.B., 2006. Strength, but not muscle mass, is associated with mortality in the health, aging and body composition study cohort. J Gerontol A Biol Sci Med Sci 61, 72-77.

Nielsen, J.B., Sinkjaer, T., 2002. Afferent feedback in the control of human gait. J Electromyogr Kinesiol 12, 213-217.

Niendam, T.A., Laird, A.R., Ray, K.L., Dean, Y.M., Glahn, D.C., Carter, C.S., 2012. Meta-analytic evidence for a superordinate cognitive control network subserving diverse executive functions. Cogn Affect Behav Neurosci 12, 241-268. doi:10.3758/s13415-011-0083-5

Nofuji, Y., Shinkai, S., Taniguchi, Y., Amano, H., Nishi, M., Murayama, H., Fujiwara, Y., Suzuki, T., 2016. Associations of Walking Speed, Grip Strength, and Standing Balance With Total and Cause-Specific Mortality in a General Population of Japanese Elders. J Am Med Dir Assoc 17, 184.e1-7. doi:10.1016/j.jamda.2015.11.003

O'Callaghan, C., Bertoux, M., Hornberger, M., 2014. Beyond and below the cortex: the contribution of striatal dysfunction to cognition and behaviour in neurodegeneration. J Neurol Neurosurg Psychiatr 85, 371-378. doi:10.1136/jnnp-2012-304558

O'Connell, M.D.L., Wu, F.C.W., 2014. Androgen effects on skeletal muscle: implications for the development and management of frailty. Asian J. Androl. 16, 203-212. doi:10.4103/1008-682X.122581

O'Driscoll, S.W., Horii, E., Ness, R., Cahalan, T.D., Richards, R.R., An, K.N., 1992. The relationship between wrist position, grasp size, and grip strength. J Hand Surg Am 17, 169-177.

Ohtsuki, T., 1981. Inhibition of individual fingers during grip strength exertion. Ergonomics 24, 21-36. doi:10.1080/00140138108924827

Olafsdottir, H., Yoshida, N., Zatsiorsky, V.M., Latash, M.L., 2007a. Elderly show decreased adjustments of motor synergies in preparation to action. Clin Biomech (Bristol, Avon) 22, 44-51. doi:10.1016/j.clinbiomech.2006.08.005

Olafsdottir, H., Yoshida, N., Zatsiorsky, V.M., Latash, M.L., 2005. Anticipatory covariation of finger forces during self-paced and reaction time force production. Neurosci Lett 381, 92-96. doi:10.1016/j.neulet.2005.02.003 
Olafsdottir, H., Zhang, W., Zatsiorsky, V.M., Latash, M.L., 2007b. Age-related changes in multifinger synergies in accurate moment of force production tasks. J Appl Physiol 102, 1490-1501. doi:10.1152/japplphysiol.00966.2006

Olafsdottir, H.B., Zatsiorsky, V.M., Latash, M.L., 2008. The effects of strength training on finger strength and hand dexterity in healthy elderly individuals. J Appl Physiol 105, 1166-1178. doi:10.1152/japplphysiol.00054.2008

Ortega, F.B., Silventoinen, K., Tynelius, P., Rasmussen, F., 2012. Muscular strength in male adolescents and premature death: cohort study of one million participants. BMJ 345, e7279. doi:10.1136/bmj.e7279

Ortuño, F., Guillen-Grima, F., López-García, P., Gómez, J., Pla, J., 2011. Functional neural networks of time perception: challenge and opportunity for schizophrenia research. Schizophr Res 125, 129-135. doi:10.1016/j.schres.2010.10.003

Ouden, den, M.E.M., Schuurmans, M.J., Arts, I.E.M.A., van der Schouw, Y.T., 2011. Physical performance characteristics related to disability in older persons: a systematic review. Maturitas 69, 208-219. doi:10.1016/j.maturitas.2011.04.008

Owen, A.M., Iddon, J.L., Hodges, J.R., Summers, B.A., Robbins, T.W., 1997. Spatial and non-spatial working memory at different stages of Parkinson's disease. Neuropsychologia 35, 519-532.

Owen, A.M., James, M., Leigh, P.N., Summers, B.A., Marsden, C.D., Quinn, N.P., Lange, K.W., Robbins, T.W., 1992. Fronto-striatal cognitive deficits at different stages of Parkinson's disease. Brain 115 ( Pt 6), 1727-1751.

Owen, A.M., McMillan, K.M., Laird, A.R., Bullmore, E., 2005. N-back working memory paradigm: a meta-analysis of normative functional neuroimaging studies. Hum Brain Mapp 25, 46-59. doi:10.1002/hbm.20131

Page, S.T., Amory, J.K., Bowman, F.D., Anawalt, B.D., Matsumoto, A.M., Bremner, W.J., Tenover, J.L., 2005. Exogenous testosterone (T) alone or with finasteride increases physical performance, grip strength, and lean body mass in older men with low serum T. J. Clin. Endocrinol. Metab. 90, 1502-1510. doi:10.1210/jc.2004-1933

Pagonabarraga, J., Kulisevsky, J., 2012. Cognitive impairment and dementia in Parkinson's disease. Neurobiol. Dis. 46, 590-596. doi:10.1016/j.nbd.2012.03.029

Parikh, P.J., Cole, K.J., 2012. Handling objects in old age: forces and moments acting on the object. Journal of Applied Physiology 112, 1095-1104. doi:10.1152/japplphysiol.01385.2011

Park, J., Lewis, M.M., Huang, X., Latash, M.L., 2013. Effects of olivo-ponto-cerebellar atrophy (OPCA) on finger interaction and coordination. Clin Neurophysiol 124, 991998. doi:10.1016/j.clinph.2012.10.021

Park, J., Sun, Y., Zatsiorsky, V.M., Latash, M.L., 2011. Age-related changes in optimality and motor variability: an example of multifinger redundant tasks. Exp Brain Res 212, 1-18. doi:10.1007/s00221-011-2692-1

Park, J., Wu, Y.H., Lewis, M.M., Huang, X., Latash, M.L., 2012. Changes in multifinger interaction and coordination in Parkinson's disease. J Neurophysiol 108, 915-924. doi:10.1152/jn.00043.2012

Peinemann, A., Lehner, C., Conrad, B., Siebner, H.R., 2001. Age-related decrease in paired-pulse intracortical inhibition in the human primary motor cortex. Neurosci Lett 313, 33-36.

Penner-Wilger, M., Anderson, M.L., 2013. The relation between finger gnosis and 
mathematical ability: why redeployment of neural circuits best explains the finding. Front Psychol 4, 877. doi:10.3389/fpsyg.2013.00877

Penner-Wilger, M., Anderson, M.L., 2011. The relation between finger gnosis and mathematical ability: Can we attribute function to cortical structure with crossdomain modeling, in:.Proceedings of the Annual Meeting of the Cognitive Science Society (Vol. 33, No. 33).

Penney, T.B., Vaitilingam, L., 2008. Imaging time. Psychology of time.

Pesenti, M., Thioux, M., Seron, X., De Volder, A., 2000. Neuroanatomical substrates of arabic number processing, numerical comparison, and simple addition: a PET study. J Cogn Neurosci 12, 461-479.

Phillips, P., 1986. Grip strength, mental performance and nutritional status as indicators of mortality risk among female geriatric patients. Age Ageing 15, 53-56. doi:10.1093/ageing/15.1.53

Pini, L., Pievani, M., Bocchetta, M., Altomare, D., Bosco, P., Cavedo, E., Galluzzi, S., Marizzoni, M., Frisoni, G.B., 2016. Brain atrophy in Alzheimer's Disease and aging. Ageing Res. Rev. 30, 25-48. doi:10.1016/j.arr.2016.01.002

Plant, D.R., Lynch, G.S., 2002. Excitation- contraction coupling and sarcoplasmic reticulum function in lechanically skinned fibres from fast skeletal muscles of aged mice. The Journal of Physiology 543, 169-176. doi:10.1113/jphysiol.2002.022418

Poeppel, D., Emmorey, K., Hickok, G., Pylkkänen, L., 2012. Towards a new neurobiology of language. J Neurosci 32, 14125-14131.

Poldrack, R.A., 2006. Can cognitive processes be inferred from neuroimaging data? Trends in Cognitive Sciences 10, 59-63. doi:10.1016/j.tics.2005.12.004

Postuma, R.B., Dagher, A., 2006. Basal ganglia functional connectivity based on a metaanalysis of 126 positron emission tomography and functional magnetic resonance imaging publications. Cereb Cortex 16, 1508-1521. doi:10.1093/cercor/bhj088

Praetorius Björk, M., Johansson, B., Hassing, L.B., 2016. I forgot when I lost my gripstrong associations between cognition and grip strength in level of performance and change across time in relation to impending death. Neurobiol Aging 38, 68-72. doi:10.1016/j.neurobiolaging.2015.11.010

Press, C., Cook, R., 2015. Beyond action-specific simulation: domain-general motor contributions to perception. Trends in Cognitive Sciences 19, 176-178. doi:10.1016/j.tics.2015.01.006

Preuschhof, C., Heekeren, H.R., Taskin, B., Schubert, T., Villringer, A., 2006. Neural correlates of vibrotactile working memory in the human brain. Journal of Neuroscience 26, 13231-13239. doi:10.1523/JNEUROSCI.2767-06.2006

Preuschhof, C., Schubert, T., Villringer, A., Heekeren, H.R., 2010. Prior Information biases stimulus representations during vibrotactile decision making. J Cogn Neurosci 22, 875-887. doi:10.1162/jocn.2009.21260

Price, C.J., 2012. A review and synthesis of the first 20 years of PET and fMRI studies of heard speech, spoken language and reading. Neuroimage 62, 816-847. doi:10.1016/j.neuroimage.2012.04.062

Prins, N.D., Scheltens, P., 2015. White matter hyperintensities, cognitive impairment and dementia: an update. Nat Rev Neurol 11, 157-165. doi:10.1038/nrneurol.2015.10

Pulvermüller, F., Fadiga, L., 2010. Active perception: sensorimotor circuits as a cortical basis for language. Nature Reviews Neuroscience 11, 351-360. doi:10.1038/nrn2811 
Purser, J.L., Kuchibhatla, M.N., Fillenbaum, G.G., Harding, T., Peterson, E.D., Alexander, K.P., 2006. Identifying frailty in hospitalized older adults with significant coronary artery disease. Journal of the American Geriatrics Society 54, 1674-1681. doi:10.1111/j.1532-5415.2006.00914.x

Radua, J., Del Pozo, N.O., Gómez, J., Guillen-Grima, F., Ortuño, F., 2014. Meta-analysis of functional neuroimaging studies indicates that an increase of cognitive difficulty during executive tasks engages brain regions associated with time perception. Neuropsychologia 58, 14-22. doi:10.1016/j.neuropsychologia.2014.03.016

Raji, M.A., Kuo, Y.-F., Snih, S.A., Markides, K.S., Kristen Peek, M., Ottenbacher, K.J., 2005. Cognitive Status, Muscle Strength, and Subsequent Disability in Older Mexican Americans. Journal of the American Geriatrics Society 53, 1462-1468. doi:10.1111/j.1532-5415.2005.53457.x

Rakic, P., 2009. Evolution of the neocortex: a perspective from developmental biology. Nature Reviews Neuroscience 10, 724-735. doi:10.1038/nrn2719

Ramnani, N., 2006. The primate cortico-cerebellar system: anatomy and function. Nat Rev Neurosci 7, 511-522. doi:10.1038/nrn1953

Rantanen, T., Guralnik, J.M., Foley, D., Masaki, K., Leveille, S., Curb, J.D., White, L., 1999. Midlife hand grip strength as a predictor of old age disability. Jama 281, 558560.

Rantanen, T., Harris, T., Leveille, S.G., Visser, M., Foley, D., Masaki, K., Guralnik, J.M., 2000. Muscle strength and body mass index as long-term predictors of mortality in initially healthy men. J Gerontol A Biol Sci Med Sci 55, M168-73.

Rantanen, T., Masaki, K., Foley, D., Izmirlian, G., White, L., Guralnik, J.M., 1998. Grip strength changes over 27 yr in Japanese-American men. J Appl Physiol 85, 20472053.

Rapp, A.M., Mutschler, D.E., Erb, M., 2012. Where in the brain is nonliteral language? A coordinate-based meta-analysis of functional magnetic resonance imaging studies. Neuroimage 63, 600-610. doi:10.1016/j.neuroimage.2012.06.022

Raz, N., Williamson, A., Gunning-Dixon, F., Head, D., Acker, J.D., 2000. Neuroanatomical and cognitive correlates of adult age differences in acquisition of a perceptual-motor skill. Microsc. Res. Tech. 51, 85-93. doi:10.1002/10970029(20001001)51:1<85::AID-JEMT9>3.0.CO;2-0

Rearick, M.P., Casares, A., Santello, M., 2003. Task-dependent modulation of multi-digit force coordination patterns. J Neurophysiol 89, 1317-1326. doi:10.1152/jn.00581.2002

Richard Ridderinkhof, K., Forstmann, B.U., Wylie, S.A., Burle, B., van den Wildenberg, W.P.M., 2011. Neurocognitive mechanisms of action control: resisting the call of the Sirens. WIREs Cogn Sci 2, 174-192. doi:10.1002/wcs.99

Richter, W., Somorjai, R., Summers, R., Jarmasz, M., Menon, R.S., Gati, J.S., Georgopoulos, A.P., Tegeler, C., Uğurbil, K., Kim, S.G., 2000. Motor area activity during mental rotation studied by time-resolved single-trial fMRI. J Cogn Neurosci 12, 310-320.

Ridan, T., Pieniążek, M., Cieślar, G., Berwecki, A., Janusz, M., Kilar, Z., 2003. Influence of Number of Trials, Grip Breadth and Elbow Position on the Maximal Grip Strength. J. Hum. Kinet 3, 73-91.

Rijk, J.M., Roos, P.R., Deckx, L., van den Akker, M., Buntinx, F., 2016. Prognostic value 
of handgrip strength in people aged 60 years and older: A systematic review and meta-analysis. Geriatr Gerontol Int 16, 5-20. doi:10.1111/ggi.12508

Rijk, J.M., Roos, P.R., Deckx, L., van den Akker, M., Buntinx, F., 2015. Prognostic value of handgrip strength in people aged 60 years and older: A systematic review and meta-analysis. Geriatr Gerontol Int. doi:10.1111/ggi.12508

Roberts, H.C., Denison, H.J., Martin, H.J., Patel, H.P., Syddall, H., Cooper, C., Sayer, A.A., 2011. A review of the measurement of grip strength in clinical and epidemiological studies: towards a standardised approach.

Roberts, H.C., Syddall, H.E., Sparkes, J., Ritchie, J., Butchart, J., Kerr, A., Cooper, C., Sayer, A.A., 2014. Grip strength and its determinants among older people in different healthcare settings. Age Ageing 43, 241-246. doi:10.1093/ageing/aft118

Robertson, D.A., Savva, G.M., Coen, R.F., Kenny, R.A., 2014. Cognitive function in the prefrailty and frailty syndrome. Journal of the American Geriatrics Society 62, 2118 2124. doi:10.1111/jgs.13111

Robertson, E.M., 2007. The serial reaction time task: implicit motor skill learning? Journal of Neuroscience 27, 10073-10075. doi:10.1523/JNEUROSCI.2747-07.2007

Robinson, J.L., Laird, A.R., Glahn, D.C., Blangero, J., Sanghera, M.K., Pessoa, L., Fox, P.M., Uecker, A., Friehs, G., Young, K.A., Griffin, J.L., Lovallo, W.R., Fox, P.T., 2012. The functional connectivity of the human caudate: an application of metaanalytic connectivity modeling with behavioral filtering. Neuroimage 60, 117-129. doi:10.1016/j.neuroimage.2011.12.010

Rockwood, K., Mitnitski, A., 2007. Frailty in relation to the accumulation of deficits. J Gerontol A Biol Sci Med Sci 62, 722-727.

Rockwood, K., Mitnitski, A., Song, X., Steen, B., Skoog, I., 2006. Long-term risks of death and institutionalization of elderly people in relation to deficit accumulation at age 70. Journal of the American Geriatrics Society 54, 975-979. doi:10.1111/j.15325415.2006.00738.x

Rogers, M.W., Kukulka, C.G., Soderberg, G.L., 1992. Age-related changes in postural responses preceding rapid self-paced and reaction time arm movements. J Gerontol 47, M159-65.

Rolland, Y., Lauwers-Cances, V., Cesari, M., Vellas, B., Pahor, M., Grandjean, H., 2006. Physical performance measures as predictors of mortality in a cohort of communitydwelling older French women. Eur J Epidemiol 21, 113-122. doi:10.1007/s10654005-5458-x

Roman, D., Mahoney, K., \& Mohamadi, A. (2013). Sarcopenia: What's in a name?. Journal of the American Medical Directors Association, 14(2), 80-82.

Romero-Ortuno, R., Kenny, R.A., 2012. The frailty index in Europeans: association with age and mortality. Age Ageing. doi:10.1093/ageing/afs051

Romo, R., de Lafuente, V., 2013. Conversion of sensory signals into perceptual decisions. Prog Neurobiol 103, 41-75. doi:10.1016/j.pneurobio.2012.03.007

Rosano, C., Studenski, S. A., Aizenstein, H. J., Boudreau, R. M., Longstreth Jr, W. T., \& Newman, A. B. (2012). Slower gait, slower information processing and smaller prefrontal area in older adults. Age and Ageing, 41(1), 58-64.

Rosenberg, I.H., 1989. Summary comments. Am. J. Clin. Nutr. 50, 1231-1233.

Rosselli, M., Ardila, A., Bernal, B., 2015. [Angular gyrus connectivity model for language: a functional neuroimaging meta-analysis]. Rev Neurol 60, 495-503. 
Rothman, M.D., Leo-Summers, L., Gill, T.M., 2008. Prognostic significance of potential frailty criteria. Journal of the American Geriatrics Society 56, 2211-2116.

doi:10.1111/j.1532-5415.2008.02008.x

Rottschy, C., Langner, R., Dogan, I., Reetz, K., Laird, A.R., Schulz, J.B., Fox, P.T., Eickhoff, S.B., 2012. Modelling neural correlates of working memory: a coordinatebased meta-analysis. Neuroimage 60, 830-846.

doi:10.1016/j.neuroimage.2011.11.050

Rueckert, L., Lange, N., Partiot, A., Appollonio, I., Litvan, I., Le Bihan, D., Grafman, J., 1996. Visualizing cortical activation during mental calculation with functional MRI. Neuroimage 3, 97-103. doi:10.1006/nimg.1996.0011

Russ, D.W., Gregg-Cornell, K., Conaway, M.J., Clark, B.C., 2012. Evolving concepts on the age-related changes in "muscle quality". J Cachexia Sarcopenia Muscle 3, 95-109. doi:10.1007/s13539-011-0054-2

Sachdev, P.S., Parslow, R., Wen, W., Anstey, K.J., Easteal, S., 2009. Sex differences in the causes and consequences of white matter hyperintensities. Neurobiol Aging 30, 946-956. doi:10.1016/j.neurobiolaging.2007.08.023

Sachdev, P.S., Wen, W., Christensen, H., Jorm, A.F., 2005. White matter hyperintensities are related to physical disability and poor motor function. J Neurol Neurosurg Psychiatr 76, 362-367. doi:10.1136/jnnp.2004.042945

Sakai, H., Takahara, M., Honjo, N.F., Doi, S., Sadato, N., Uchiyama, Y., 2012. Regional frontal gray matter volume associated with executive function capacity as a risk factor for vehicle crashes in normal aging adults. PLoS ONE 7, e45920. doi:10.1371/journal.pone.0045920

Salthouse, T.A., Babcock, R.L., 1991. Decomposing adult age differences in working memory. Developmental Psychology 27, 763.

Samuel, D., Rowe, P., 2012. An investigation of the association between grip strength and hip and knee joint moments in older adults. Arch Gerontol Geriatr 54, 357-360. doi:10.1016/j.archger.2011.03.009

Santello, M., Baud-Bovy, G., Jörntell, H., 2013. Neural bases of hand synergies. Front Comput Neurosci 7, 23. doi:10.3389/fncom.2013.00023

Santos, L., Cyrino, E. S., Antunes, M., Santos, D. A., \& Sardinha, L. B. (2017). Sarcopenia and physical independence in older adults: the independent and synergic role of muscle mass and muscle function. Journal of cachexia, sarcopenia and muscle, 8(2), 245-250.

Sasaki, H., Kasagi, F., Yamada, M., Fujita, S., 2007. Grip strength predicts cause-specific mortality in middle-aged and elderly persons. Am. J. Med. 120, 337-342. doi:10.1016/j.amjmed.2006.04.018

Sasaoka, T., Mizuhara, H., Inui, T., 2014. Dynamic parieto-premotor network for mental image transformation revealed by simultaneous EEG and fMRI measurement. J Cogn Neurosci 26, 232-246. doi:10.1162/jocn_a_00493

Sayer, A.A., 2006. Is grip strength associated with health-related quality of life? Findings from the Hertfordshire Cohort Study. Age Ageing 35, 409-415. doi:10.1093/ageing/afl024

Sayer, A.A., Syddall, H., Martin, H., Patel, H., Baylis, D., Cooper, C., 2008. The developmental origins of sarcopenia. J Nutr Health Aging 12, 427-432.

Sánchez-Cubillo, I., Periáñez, J.A., Adrover-Roig, D., Rodríguez-Sánchez, J.M., Ríos- 
Lago, M., Tirapu, J., Barceló, F., 2009. Construct validity of the Trail Making Test: role of task-switching, working memory, inhibition/interference control, and visuomotor abilities. J Int Neuropsychol Soc 15, 438-450. doi:10.1017/S1355617709090626

Scaglioni, G., Ferri, A., Minetti, A.E., Martin, A., Van Hoecke, J., Capodaglio, P., Sartorio, A., Narici, M.V., 2002. Plantar flexor activation capacity and H reflex in older adults: adaptations to strength training. J Appl Physiol 92, 2292-2302. doi:10.1152/japplphysiol.00367.2001

Schmahmann, J. D., \& Pandya, D. N. (2014). Neuroanatomy and Behavioral Neurology of Subcortical Systems. In Dementia: Comprehensive Principles and Practices, (pages 32-69). Edited by Bradford Dickerson, and Alireza Atri, Oxford University Press.

Schubotz, R., 1999. Instruction differentiates the processing of temporal and spatial sequential patterns: evidence from slow wave activity in humans. Neurosci Lett 265 , $1-4$.

Schubotz, R.I., 2007. Prediction of external events with our motor system: towards a new framework. Trends in Cognitive Sciences 11, 211-218.

doi:10.1016/j.tics.2007.02.006

Schubotz, R.I., 2004. Human premotor cortex: Beyond motor performance. Max Planck Institute for Human Cognitive and Brain Sciences Leipzig.

Schubotz, R.I., Cramon, von, D.Y., 2003. Functional-anatomical concepts of human premotor cortex: evidence from fMRI and PET studies. Neuroimage 20 Suppl 1, S120-31.

Schubotz, R.I., Cramon, von, D.Y., 2002a. A blueprint for target motion: fMRI reveals perceived sequential complexity to modulate premotor cortex. Neuroimage 16, 920935.

Schubotz, R.I., Cramon, von, D.Y., 2002b. Predicting perceptual events activates corresponding motor schemes in lateral premotor cortex: an fMRI study. Neuroimage 15, 787-796. doi:10.1006/nimg.2001.1043

Schubotz, R.I., Cramon, von, D.Y., 2001a. Functional organization of the lateral premotor cortex: fMRI reveals different regions activated by anticipation of object properties, location and speed. Cognitive Brain Research.

Schubotz, R.I., Cramon, von, D.Y., 2001b. Interval and ordinal properties of sequences are associated with distinct premotor areas. Cereb Cortex 11, 210-222.

Schubotz, R.I., Cramon, von, D.Y., Lohmann, G., 2003. Auditory what, where, and when: a sensory somatotopy in lateral premotor cortex. Neuroimage 20, 173-185. doi:10.1016/S1053-8119(03)00218-0

Schubotz, R.I., Yves von Cramon, D., 2002. Dynamic patterns make the premotor cortex interested in objects: influence of stimulus and task revealed by fMRI. Brain Res Cogn Brain Res 14, 357-369.

Schulze, K., Vargha-Khadem, F., Mishkin, M., 2012. Test of a motor theory of long-term auditory memory. Proceedings of the National Academy of Sciences 109, 7121-7125. doi:10.1073/pnas.1204717109

Scimeca, J.M., Badre, D., 2012. Striatal contributions to declarative memory retrieval. Neuron 75, 380-392. doi:10.1016/j.neuron.2012.07.014

Scott, S.K., McGettigan, C., Eisner, F., 2009. A little more conversation, a little less 
action--candidate roles for the motor cortex in speech perception. Nature Reviews Neuroscience 10, 295-302. doi:10.1038/nrn2603

Scripture, E., Smith, T., Brown, E., 1894. On the education of muscular control and power. Stud Yale Psychol Lab 2, 114-119.

Searle, S.D., Mitnitski, A., Gahbauer, E.A., Gill, T.M., Rockwood, K., 2008. A standard procedure for creating a frailty index. BMC Geriatr 8, 24. doi:10.1186/1471-2318-824

Seeley, W.W., Crawford, R.K., Zhou, J., Miller, B.L., Greicius, M.D., 2009.

Neurodegenerative diseases target large-scale human brain networks. Neuron 62, $42-$ 52. doi:10.1016/j.neuron.2009.03.024

Seidler, R., Erdeniz, B., Koppelmans, V., Hirsiger, S., Mérillat, S., Jäncke, L., 2015. Associations between age, motor function, and resting state sensorimotor network connectivity in healthy older adults. Neuroimage 108, 47-59. doi:10.1016/j.neuroimage.2014.12.023

Seidler, R.D., Bernard, J.A., Burutolu, T.B., Fling, B.W., Gordon, M.T., Gwin, J.T., Kwak, Y., Lipps, D.B., 2010. Motor control and aging: links to age-related brain structural, functional, and biochemical effects. Neuroscience and Biobehavioral Reviews 34, 721-733. doi:10.1016/j.neubiorev.2009.10.005

Shechtman, O., Mann, W.C., Justiss, M.D., Tomita, M., 2004. Grip Strength in the Frail Elderly. Am J Phys Med Rehabil 83, 819.

Shepard, R.N., Metzler, J., 1971. Mental rotation of three-dimensional objects. Science 171, 701-703.

Shields, R.K., Leo, K.C., Messaros, A.J., Somers, V.K., 1999. Effects of repetitive handgrip training on endurance, specificity, and cross-education. Phys Ther 79, 467475.

Shim, J.K., Lay, B.S., Zatsiorsky, V.M., Latash, M.L., 2004. Age-related changes in finger coordination in static prehension tasks. J Appl Physiol 97, 213-224. doi:10.1152/japplphysiol.00045.2004

Shinohara, M., Latash, M.L., Zatsiorsky, V.M., 2003a. Age effects on force produced by intrinsic and extrinsic hand muscles and finger interaction during MVC tasks. J Appl Physiol 95, 1361-1369. doi:10.1152/japplphysiol.00070.2003

Shinohara, M., Li, S., Kang, N., Zatsiorsky, V.M., Latash, M.L., 2003b. Effects of age and gender on finger coordination in MVC and submaximal force-matching tasks. $\mathrm{J}$ Appl Physiol 94, 259-270. doi:10.1152/japplphysiol.00643.2002

Shinohara, M., Scholz, J.P., Zatsiorsky, V.M., Latash, M.L., 2004. Finger interaction during accurate multi-finger force production tasks in young and elderly persons. Exp Brain Res 156, 282-292. doi:10.1007/s00221-003-1786-9

Siegel, M., Engel, A.K., Donner, T.H., 2011. Cortical network dynamics of perceptual decision-making in the human brain. Front. Hum. Neurosci. 5, 21. doi:10.3389/fnhum.2011.00021

Simon, S.R., Meunier, M., Piettre, L., Berardi, A.M., Segebarth, C.M., Boussaoud, D., 2002. Spatial attention and memory versus motor preparation: premotor cortex involvement as revealed by fMRI. J Neurophysiol 88, 2047-2057.

Sjöbeck, M., Dahlén, S., Englund, E., 1999. Neuronal loss in the brainstem and cerebellum--part of the normal aging process? A morphometric study of the vermis cerebelli and inferior olivary nucleus. J Gerontol A Biol Sci Med Sci 54, B363-8. 
Snih, Al, S., Markides, K.S., Ray, L., Ostir, G.V., Goodwin, J.S., 2002. Handgrip Strength and Mortality in Older Mexican Americans. Journal of the American Geriatrics Society 50, 1250-1256. doi:10.1046/j.1532-5415.2002.50312.x

Snyder, P.J., Peachey, H., Hannoush, P., Berlin, J.A., Loh, L., Lenrow, D.A., Holmes, J.H., Dlewati, A., Santanna, J., Rosen, C.J., Strom, B.L., 1999. Effect of testosterone treatment on body composition and muscle strength in men over 65 years of age. J. Clin. Endocrinol. Metab. 84, 2647-2653. doi:10.1210/jcem.84.8.5885

Solnik, S., Zatsiorsky, V.M., Latash, M.L., 2014. Internal forces during static prehension: effects of age and grasp configuration. Journal of Motor Behavior 46, 211-222. doi:10.1080/00222895.2014.881315

Spaniol, J., Davidson, P.S.R., Kim, A.S.N., Han, H., Moscovitch, M., Grady, C.L., 2009. Event-related fMRI studies of episodic encoding and retrieval: meta-analyses using activation likelihood estimation. Neuropsychologia 47, 1765-1779. doi:10.1016/j.neuropsychologia.2009.02.028

Speed, C.A., Campbell, R., 2012. Mechanisms of strength gain in a handgrip exercise programme in rheumatoid arthritis. Rheumatol. Int. 32, 159-163. doi:10.1007/s00296-010-1596-X

Srinivas-Shankar, U., Roberts, S.A., Connolly, M.J., O'Connell, M.D.L., Adams, J.E., Oldham, J.A., Wu, F.C.W., 2010. Effects of testosterone on muscle strength, physical function, body composition, and quality of life in intermediate-frail and frail elderly men: a randomized, double-blind, placebo-controlled study. J. Clin. Endocrinol. Metab. 95, 639-650. doi:10.1210/jc.2009-1251

Steele, J., Ferrari, P.F., Fogassi, L., 2012. From action to language: comparative perspectives on primate tool use, gesture and the evolution of human language. Philos Trans R Soc Lond, B, Biol Sci 367, 4-9. doi:10.1098/rstb.2011.0295

Steiber, N., 2016. Strong or Weak Handgrip? Normative Reference Values for the German Population across the Life Course Stratified by Sex, Age, and Body Height. PLoS ONE 11, e0163917. doi:10.1371/journal.pone.0163917

Stelmach, G.E., Populin, L., Müller, F., 1990. Postural muscle onset and voluntary movement in the elderly. Neurosci Lett 117, 188-193.

Sternäng, O., Reynolds, C.A., Finkel, D., Ernsth-Bravell, M., Pedersen, N.L., Dahl Aslan, A.K., 2015. Grip Strength and Cognitive Abilities: Associations in Old Age. J Gerontol B Psychol Sci Soc Sci. doi:10.1093/geronb/gbv017

Stevens, J.E., Stackhouse, S.K., Binder-Macleod, S.A., Snyder-Mackler, L., 2003. Are voluntary muscle activation deficits in older adults meaningful? Muscle Nerve 27, 99-101. doi:10.1002/mus.10279

Stijntjes, M., Aartsen, M.J., Taekema, D.G., Gussekloo, J., Huisman, M., Meskers, C.G.M., de Craen, A.J.M., Maier, A.B., 2016. Temporal Relationship Between Cognitive and Physical Performance in Middle-Aged to Oldest Old People. J

Gerontol A Biol Sci Med Sci. doi:10.1093/gerona/glw133

Stocco, A., Lebiere, C., Anderson, J.R., 2010. Conditional routing of information to the cortex: a model of the basal ganglia's role in cognitive coordination. Psychol Rev 117, 541-574. doi:10.1037/a0019077

Stoodley, C.J., 2012. The cerebellum and cognition: evidence from functional imaging studies. Cerebellum 11, 352-365. doi:10.1007/s12311-011-0260-7

Stoodley, C.J., Schmahmann, J.D., 2009. Functional topography in the human 
cerebellum: a meta-analysis of neuroimaging studies. Neuroimage 44, 489-501. doi:10.1016/j.neuroimage.2008.08.039

Strand, B.H., Cooper, R., Bergland, A., Jørgensen, L., Schirmer, H., Skirbekk, V., Emaus, N., 2016. The association of grip strength from midlife onwards with all-cause and cause-specific mortality over 17 years of follow-up in the Tromsø Study. J Epidemiol Community Health. doi:10.1136/jech-2015-206776

Stromberg, B.V., 1986. Contralateral therapy in upper extremity rehabilitation. Am J Phys Med 65, 135-143.

Studenski, S.A., Peters, K.W., Alley, D.E., Cawthon, P.M., McLean, R.R., Harris, T.B., Ferrucci, L., Guralnik, J.M., Fragala, M.S., Kenny, A.M., Kiel, D.P., Kritchevsky, S.B., Shardell, M.D., Dam, T.-T.L., Vassileva, M.T., 2014. The FNIH sarcopenia project: rationale, study description, conference recommendations, and final estimates. J Gerontol A Biol Sci Med Sci 69, 547-558. doi:10.1093/gerona/glu010

Stuss, D.T., 2011a. Traumatic brain injury: relation to executive dysfunction and the frontal lobes. Curr Opin Neurol 24, 584-589. doi:10.1097/WCO.0b013e32834c7eb9

Stuss, D.T., 2011b. Functions of the frontal lobes: relation to executive functions. J Int Neuropsychol Soc 17, 759-765. doi:10.1017/S1355617711000695

Syddall, H., Cooper, C., Martin, F., Briggs, R., Aihie Sayer, A., 2003. Is grip strength a useful single marker of frailty? Age Ageing 32, 650-656.

Taekema, D.G., Gussekloo, J., Maier, A.B., Westendorp, R.G.J., de Craen, A.J.M., 2010. Handgrip strength as a predictor of functional, psychological and social health. A prospective population-based study among the oldest old. Age Ageing 39, 331-337. doi:10.1093/ageing/afq022

Taekema, D.G.D., Ling, C.H.Y.C., Kurrle, S.E.S., Cameron, I.D.I., Meskers, C.G.M.C., Blauw, G.J.G., Westendorp, R.G.J.R., de Craen, A.J.M.A., Maier, A.B.A., 2012. Temporal relationship between handgrip strength and cognitive performance in oldest old people. Age Ageing 41, 506-512. doi:10.1093/ageing/afs013

Tanaka, S., Honda, M., Sadato, N., 2005. Modality-specific cognitive function of medial and lateral human Brodmann area 6. Journal of Neuroscience 25, 496-501. doi:10.1523/JNEUROSCI.4324-04.2005

Taylor, P.C.J., Nobre, A.C., Rushworth, M.F.S., 2007. Subsecond changes in top down control exerted by human medial frontal cortex during conflict and action selection: a combined transcranial magnetic stimulation electroencephalography study. Journal of Neuroscience 27, 11343-11353. doi:10.1523/JNEUROSCI.2877-07.2007

Tenover, J.S., 1992. Effects of testosterone supplementation in the aging male. J. Clin. Endocrinol. Metab. 75, 1092-1098. doi:10.1210/jcem.75.4.1400877

Theou, O., Jones, G.R., Jakobi, J.M., Mitnitski, A., Vandervoort, A.A., 2011. A comparison of the relationship of 14 performance-based measures with frailty in older women. Appl Physiol Nutr Metab 36, 928-938. doi:10.1139/h11-116

Tian, Q., Chastan, N., Bair, W. N., Resnick, S. M., Ferrucci, L., \& Studenski, S. A. (2017). The brain map of gait variability in aging, cognitive impairment and dementia - a systematic review. Neuroscience \& Biobehavioral Reviews, 74, 149162.

Tieland, M., Trouwborst, I., Clark, B.C., 2018. Skeletal muscle performance and ageing. J Cachexia Sarcopenia Muscle 9, 3-19. doi:10.1002/jcsm.12238

Tombaugh, T.N., 2004. Trail Making Test A and B: normative data stratified by age and 
education. Arch Clin Neuropsychol 19, 203-214. doi:10.1016/S08876177(03)00039-8

Travison, T.G., Basaria, S., Storer, T.W., Jette, A.M., Miciek, R., Farwell, W.R., Choong, K., Lakshman, K., Mazer, N.A., Coviello, A.D., Knapp, P.E., Ulloor, J., Zhang, A., Brooks, B., Nguyen, A.-H., Eder, R., LeBrasseur, N., Elmi, A., Appleman, E., HedeBrierley, L., Bhasin, G., Bhatia, A., Lazzari, A., Davis, S., Ni, P., Collins, L., Bhasin, S., 2011. Clinical meaningfulness of the changes in muscle performance and physical function associated with testosterone administration in older men with mobility limitation. J Gerontol A Biol Sci Med Sci 66, 1090-1099. doi:10.1093/gerona/glr100

Tsukiura, T., Fujii, T., Takahashi, T., Xiao, R., Inase, M., Iijima, T., Yamadori, A., Okuda, J., 2001. Neuroanatomical discrimination between manipulating and maintaining processes involved in verbal working memory; a functional MRI study. Brain Res Cogn Brain Res 11, 13-21.

Tubiana, R., 1981a. Architecture and functions of the hand, in:. WB Saunders, Philadelphia, pp. 19-93.

Tubiana, R., 1981b. The hand, vol. 1. Philadelphia, Penn.: Sanders.

Ulfhake, B., Bergman, E., Edstrom, E., Fundin, B.T., Johnson, H., Kullberg, S., Ming, Y., 2000. Regulation of neurotrophin signaling in aging sensory and motoneurons: dissipation of target support? Mol. Neurobiol. 21, 109-135. doi: $10.1385 / \mathrm{MN}: 21: 3: 109$

Uttal, W.R., 2013. Reliability in Cognitive Neuroscience. MIT Press.

Uttal, W.R., 2001. The new phrenology: The limits of localizing cognitive processes in the brain. The MIT press, Cambridge, MA.

Vahdat, S., Darainy, M., Ostry, D.J., 2014. Structure of plasticity in human sensory and motor networks due to perceptual learning. Journal of Neuroscience 34, 2451-2463. doi:10.1523/JNEUROSCI.4291-13.2014

van Gaal, S., Scholte, H.S., Lamme, V.A.F., Fahrenfort, J.J., Ridderinkhof, K.R., 2011. Pre-SMA graymatter density predicts individual differences in action selection in the face of conscious and unconscious response conflict. J Cogn Neurosci 23, 382-390. doi:10.1162/jocn.2010.21444

van Iersel, M.B., Rikkert, M.G.M.O., 2006. Frailty criteria give heterogeneous results when applied in clinical practice. Journal of the American Geriatrics Society 54, 728-729. doi:10.1111/j.1532-5415.2006.00668_14.x

van Kan, G.A., Rolland, Y., Bergman, H., Morley, J.E., Kritchevsky, S.B., Vellas, B., 2008. The I.A.N.A Task Force on frailty assessment of older people in clinical practice. J Nutr Health Aging 12, 29-37.

Venkatraman, V., Ansari, D., Chee, M.W.L., 2005. Neural correlates of symbolic and non-symbolic arithmetic. Neuropsychologia 43, 744-753. doi:10.1016/j.neuropsychologia.2004.08.005

Verghese, J., Wang, C., Lipton, R. B., \& Holtzer, R. (2012). Motoric cognitive risk syndrome and the risk of dementia. Journals of Gerontology Series A: Biomedical Sciences and Medical Sciences, 68(4), 412-418.

Verleger, R., Kuniecki, M., Möller, F., Fritzmannova, M., Siebner, H.R., 2009. On how the motor cortices resolve an inter-hemispheric response conflict: an event-related EEG potential-guided TMS study of the flankers task. Eur J Neurosci 30, 318-326. doi:10.1111/j.1460-9568.2009.06817.x 
Vermeulen, J., Neyens, J.C.L., van Rossum, E., Spreeuwenberg, M.D., de Witte, L.P., 2011. Predicting ADL disability in community-dwelling elderly people using physical frailty indicators: a systematic review. BMC Geriatr 11, 33. doi:10.1186/1471-2318-11-33

Vingerhoets, G., de Lange, F.P., Vandemaele, P., Deblaere, K., Achten, E., 2002. Motor imagery in mental rotation: an fMRI study. Neuroimage 17, 1623-1633.

Wang, L., Larson, E.B., Bowen, J.D., van Belle, G., 2006. Performance-Based Physical Function and Future Dementia in Older People. Arch. Intern. Med. 166, 1115. doi:10.1001/archinte.166.10.1115

Wechsler, D., 1981. WAIS-R manual: Wechsler adult intelligence scale-revised. Psychological Corporation.

Weisberg, J., van Turennout, M., Martin, A., 2007. A neural system for learning about object function. Cereb Cortex 17, 513-521. doi:10.1093/cercor/bhj176

Weiss, E.J., Flanders, M., 2004. Muscular and postural synergies of the human hand. J Neurophysiol 92, 523-535. doi:10.1152/jn.01265.2003

Wennberg, A. M., Savica, R., \& Mielke, M. M. (2017). Association between Various Brain Pathologies and Gait Disturbance. Dementia and geriatric cognitive disorders, 43(3-4), 128-143.

Westerholz, J., Schack, T., Koester, D., 2013. Event-related brain potentials for goalrelated power grips. PLoS ONE 8, e68501. doi:10.1371/journal.pone.0068501

Wiener, M., Turkeltaub, P., Coslett, H.B., 2010. The image of time: a voxel-wise metaanalysis. Neuroimage 49, 1728-1740. doi:10.1016/j.neuroimage.2009.09.064

Wilson, R.S., Segawa, E., Buchman, A.S., Boyle, P.A., Hizel, L.P., Bennett, D.A., 2012. Terminal decline in motor function. Psychol Aging 27, 998-1007. doi:10.1037/a0028182

Windhorst, U., Burke, R., Dieringer, N., Evinger, C., Feldman, A., Hasan, Z., Humphrey, D., Freund, H.-J., 1991. What are the outputs of motor behavior and how are they controlled?, Motor Control: Concepts and Issues.

Wittert, G.A., Chapman, I.M., Haren, M.T., Mackintosh, S., Coates, P., Morley, J.E., 2003. Oral testosterone supplementation increases muscle and decreases fat mass in healthy elderly males with low-normal gonadal status. J Gerontol A Biol Sci Med Sci $58,618-625$.

Woodruff-Pak, D.S., Vogel, R.W., Ewers, M., Coffey, J., Boyko, O.B., Lemieux, S.K., 2001. MRI-assessed volume of cerebellum correlates with associative learning. Neurobiol Learn Mem 76, 342-357. doi:10.1006/nlme.2001.4026

Woollacott, M.H., Manchester, D.L., 1993. Anticipatory postural adjustments in older adults: are changes in response characteristics due to changes in strategy?

Wölwer, W., Stroth, S., Brinkmeyer, J., Gaebel, W., 2012. Electrophysiological correlates of planning and monitoring in first episode schizophrenia. Psychiatry Res 203, 83-88. doi:10.1016/j.pscychresns.2011.11.009

Wu, Y., Wang, W., Liu, T., Zhang, D., 2017. Association of Grip Strength With Risk of All-Cause Mortality, Cardiovascular Diseases, and Cancer in Community-Dwelling Populations: A Meta-analysis of Prospective Cohort Studies. J Am Med Dir Assoc 18, 551.e17-551.e35. doi:10.1016/j.jamda.2017.03.011

Xue, Q.-L., Bandeen-Roche, K., Varadhan, R., Zhou, J., Fried, L.P., 2008. Initial manifestations of frailty criteria and the development of frailty phenotype in the 
Women's Health and Aging Study II. J Gerontol A Biol Sci Med Sci 63, 984-990.

Xue, Q.-L., Guralnik, J.M., Beamer, B.A., Fried, L.P., Chaves, P.H.M., 2015. Monitoring 6-month trajectory of grip strength improves the prediction of long-term change in grip strength in disabled older women. J Gerontol A Biol Sci Med Sci 70, 367-373. doi:10.1093/gerona/glu149

Xue, Q.-L., Walston, J.D., Fried, L.P., Beamer, B.A., 2011. Prediction of risk of falling, physical disability, and frailty by rate of decline in grip strength: the women's health and aging study. Arch. Intern. Med. 171, 1119-1121.

doi:10.1001/archinternmed.2011.252

Yang, J., Shu, H., 2016. Involvement of the Motor System in Comprehension of NonLiteral Action Language: A Meta-Analysis Study. Brain Topography 29, 94-107. doi:10.1007/s10548-015-0427-5

Yassuda, M.S., Lopes, A., Cachioni, M., Falcao, D.V.S., Batistoni, S.S.T., Guimaraes, V.V., Neri, A.L., 2012. Frailty criteria and cognitive performance are related: data from the FIBRA study in Ermelino Matarazzo, São Paulo, Brazil. J Nutr Health Aging 16, 55-61.

Yasuda, Y., Miyamura, M., 1983. Cross transfer effects of muscular training on blood flow in the ipsilateral and contralateral forearms. European Journal of Applied Physiology 51, 321-329. doi:10.1007/BF00429068

Yu, W.S., van Duinen, H., Gandevia, S.C., 2010. Limits to the control of the human thumb and fingers in flexion and extension. J Neurophysiol 103, 278-289. doi:10.1152/jn.00797.2009

Zacks, J.M., 2008. Neuroimaging studies of mental rotation: a meta-analysis and review. J Cogn Neurosci 20, 1-19. doi:10.1162/jocn.2008.20013

Zago, L., Pesenti, M., Mellet, E., Crivello, F., Mazoyer, B., Tzourio-Mazoyer, N., 2001. Neural correlates of simple and complex mental calculation. Neuroimage 13, 314327. doi:10.1006/nimg.2000.0697

Zakzanis, K.K., Mraz, R., Graham, S.J., 2005. An fMRI study of the Trail Making Test. Neuropsychologia 43, 1878-1886. doi:10.1016/j.neuropsychologia.2005.03.013

Zarahn, E., Rakitin, B., Abela, D., Flynn, J., Stern, Y., 2005. Positive evidence against human hippocampal involvement in working memory maintenance of familiar stimuli. Cereb Cortex 15, 303-316. doi:10.1093/cercor/bhh132

Zhang, W., Sainburg, R.L., Zatsiorsky, V.M., Latash, M.L., 2006. Hand dominance and multi-finger synergies. Neurosci Lett 409, 200-204. doi:10.1016/j.neulet.2006.09.048

Zurawska Vel Grajewska, B., Sim, E.-J., Hoenig, K., Herrnberger, B., Kiefer, M., 2011. Mechanisms underlying flexible adaptation of cognitive control: behavioral and neuroimaging evidence in a flanker task. BRAIN RESEARCH 1421, 52-65. doi:10.1016/j.brainres.2011.09.022 


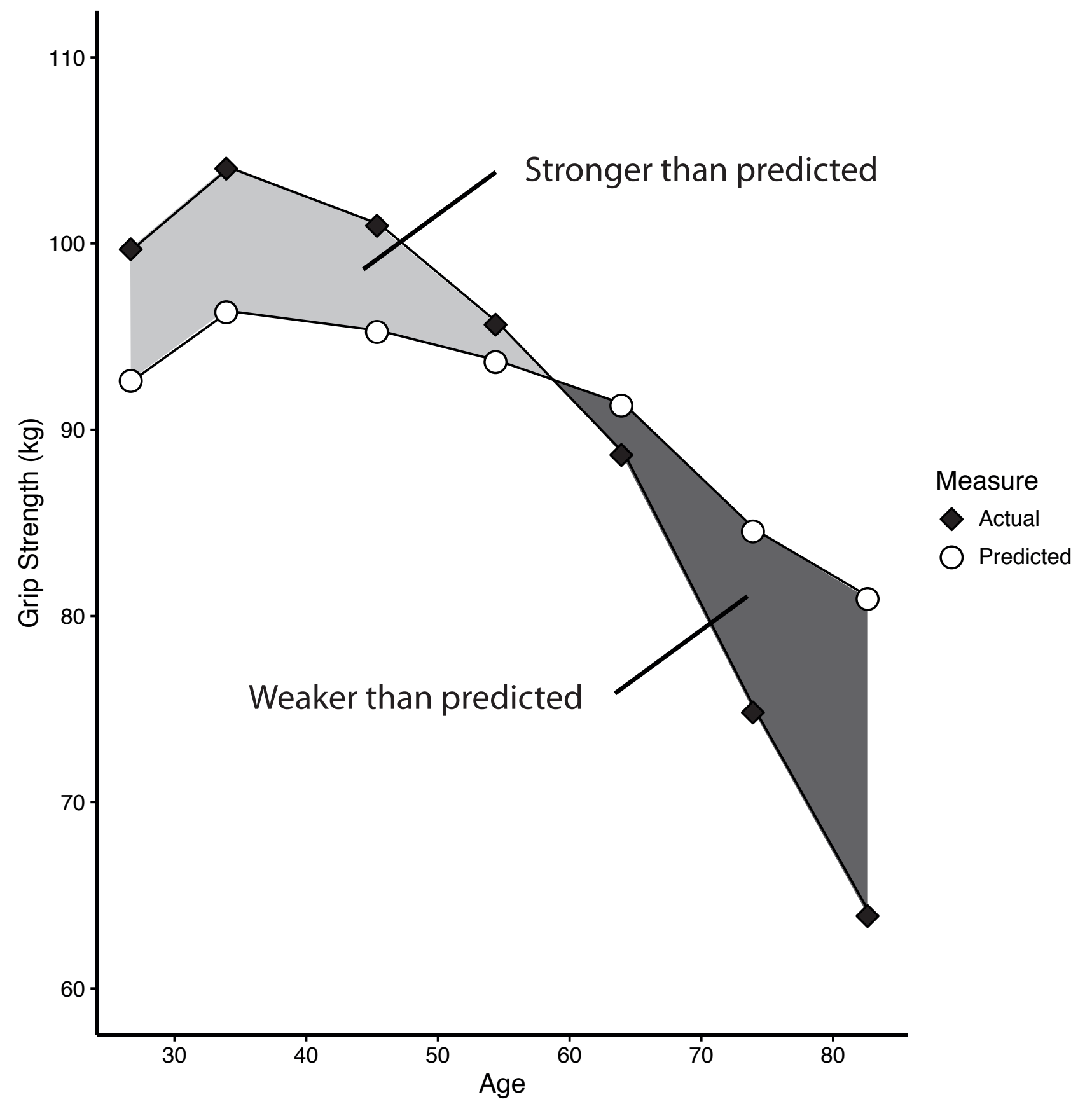

Cross sectional comparison of decade means of actual grip strength and grip strength as predicted by forearm circumference $(n=608)$. This original plot by the author was generated using data extracted from Kallman et al. (1990). These data serve to emphasise that with increasing age (above $\approx 60$ years), peripheral morphology - as represented in this case by forearm circumference, is an increasingly poor predictor of maximum grip strength. 


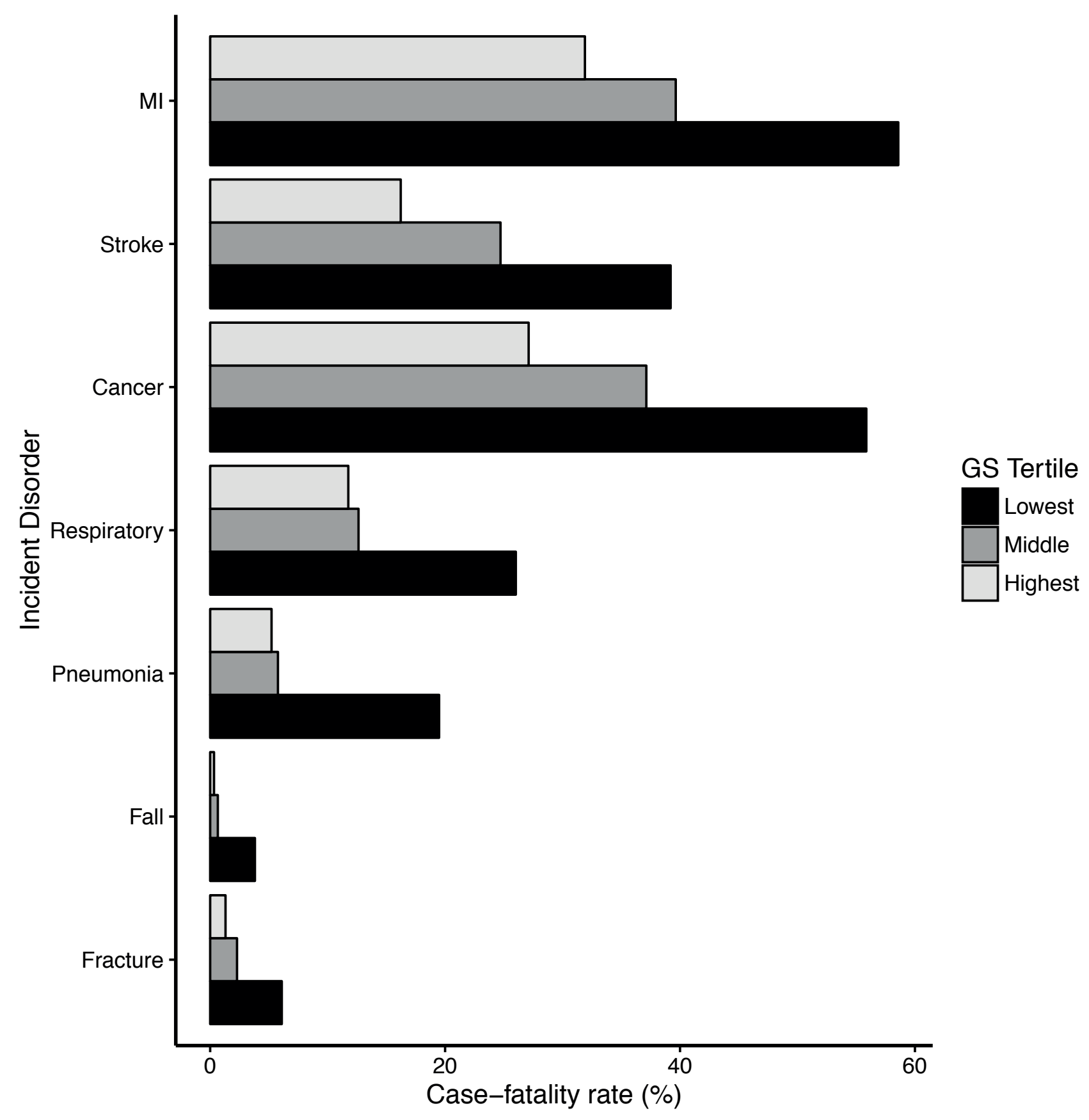

Case-fatality rates for incident cases of myocardial infarction (MI), stroke, cancer, hospital admission for pneumonia or chronic obstructive pulmonary disease, pneumonia, injury from a fall, and fracture, stratified by grip strength tertile. This original plot by the author was generated using data from the Prospective Urban-Rural Epidemiology (PURE) study ( $n=139,691)$, which were extracted from Leong et al. (2015). The pattern of variation indicates that with respect to all causes of death, the mortality rate is greatest for the group with the lowest grip strength, and smallest for the group with the highest grip strength. 


\section{Figure 3}

Self-reported Difficulty

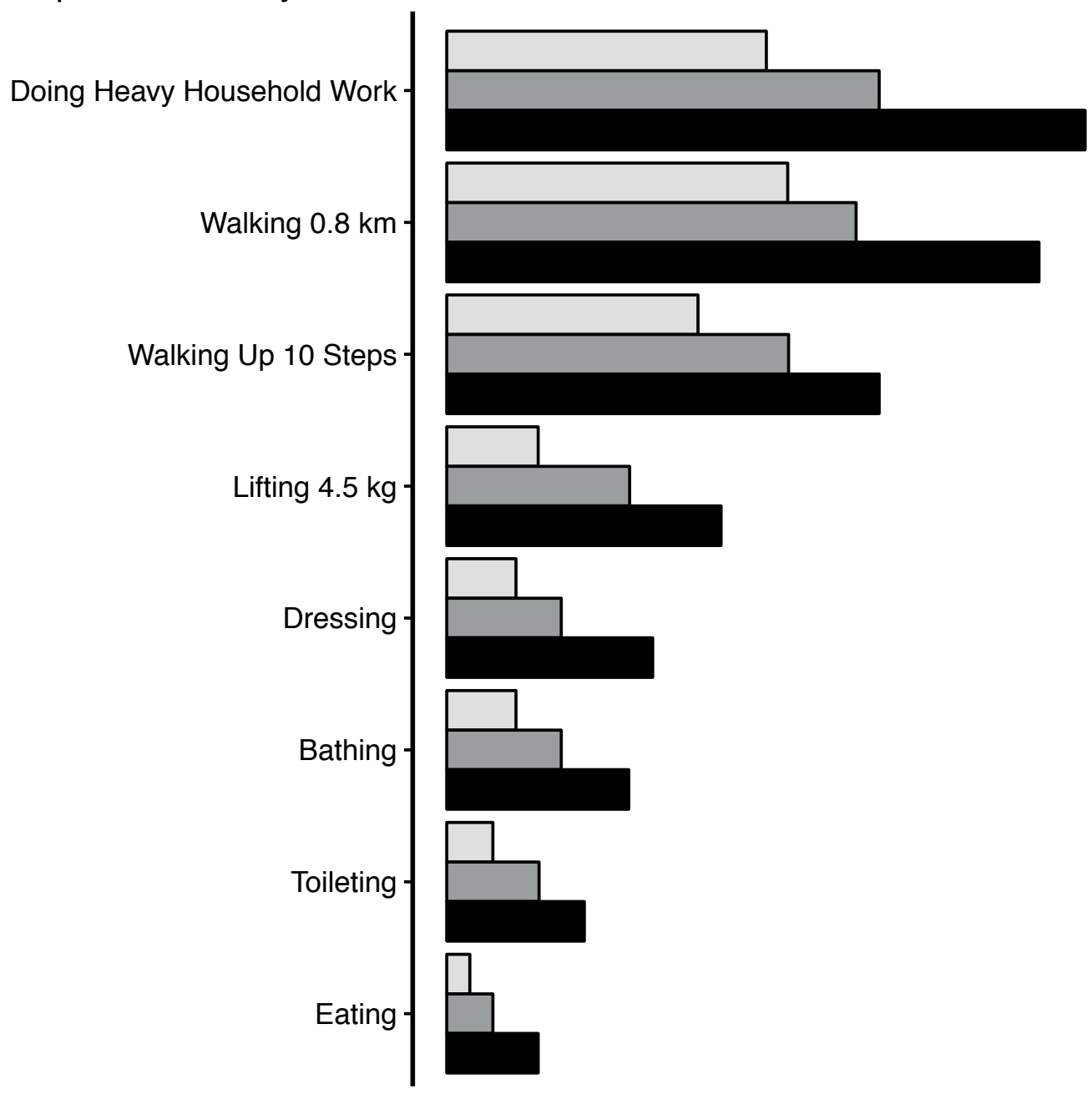

GS Tertile Lowest Middle Highest

Functional Limitations

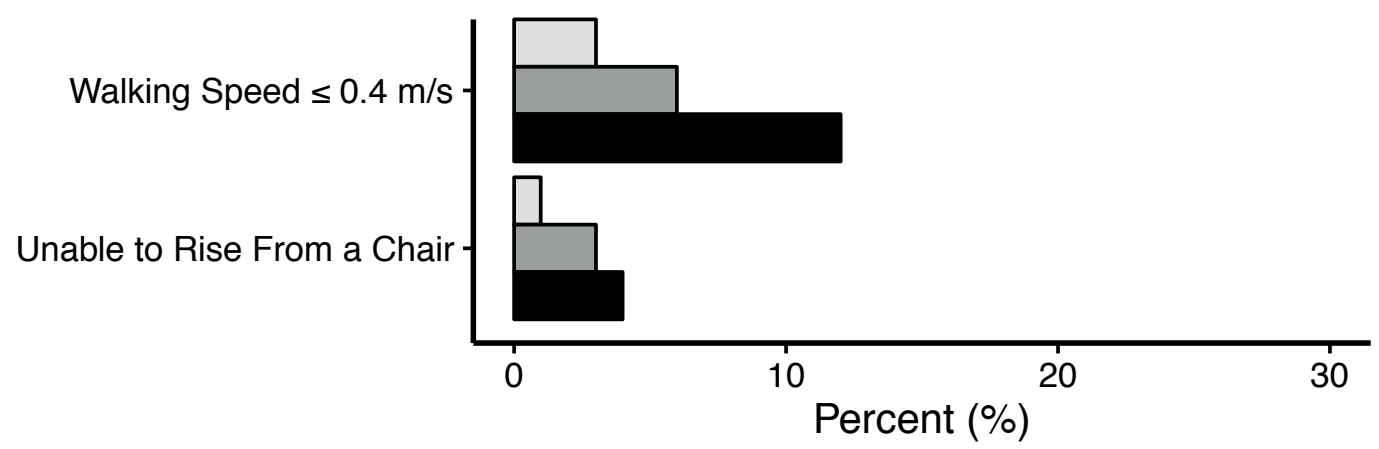

Proportion of participants with functional limitations and disability according to baseline (1965-1970) grip strength tertiles in 3218 initially healthy 45- to 68-year-old men at examination 4 - conducted from 1991-1993. This original plot by the author was generated using data extracted from Rantanen et al. (1999). The pattern of variation indicates that with respect to all forms of functional limitation and disability, the rate of incidence is greatest for the group with the lowest grip strength, and smallest for the group with the highest grip strength. 


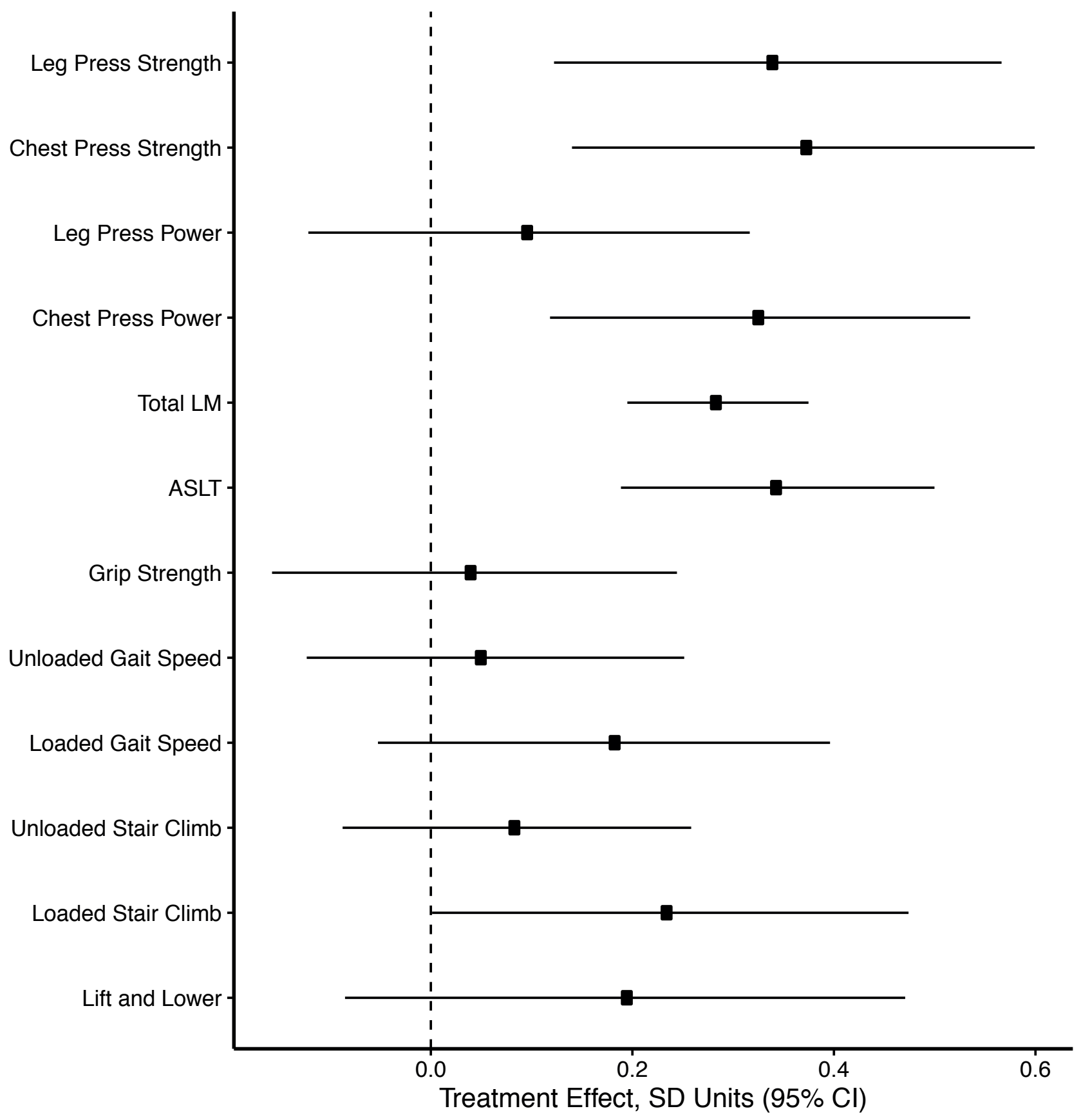

Men aged 65 years and older were randomized to placebo $(n=83)$ or $10 \mathrm{~g}$ testosterone gel daily $(n=82)$ for 6 months. Absolute treatment differences (testosterone vs. placebo arms) are plotted for the primary and secondary outcomes in units normalized to the baseline standard deviation of measurement. The point estimates (black squares) are accompanied by error bars, which correspond to 95\% confidence intervals. ALST - appendicular lean soft tissue; LM lean body mass. This original plot by the author was generated using data extracted from Travison et al. (2011). It is readily appreciated that six months of testosterone treatment ( $10 \mathrm{~g}$ daily) failed to induce reliable increases in grip strength, or in any measure of gait sufficiency. 


\section{Figure 5}

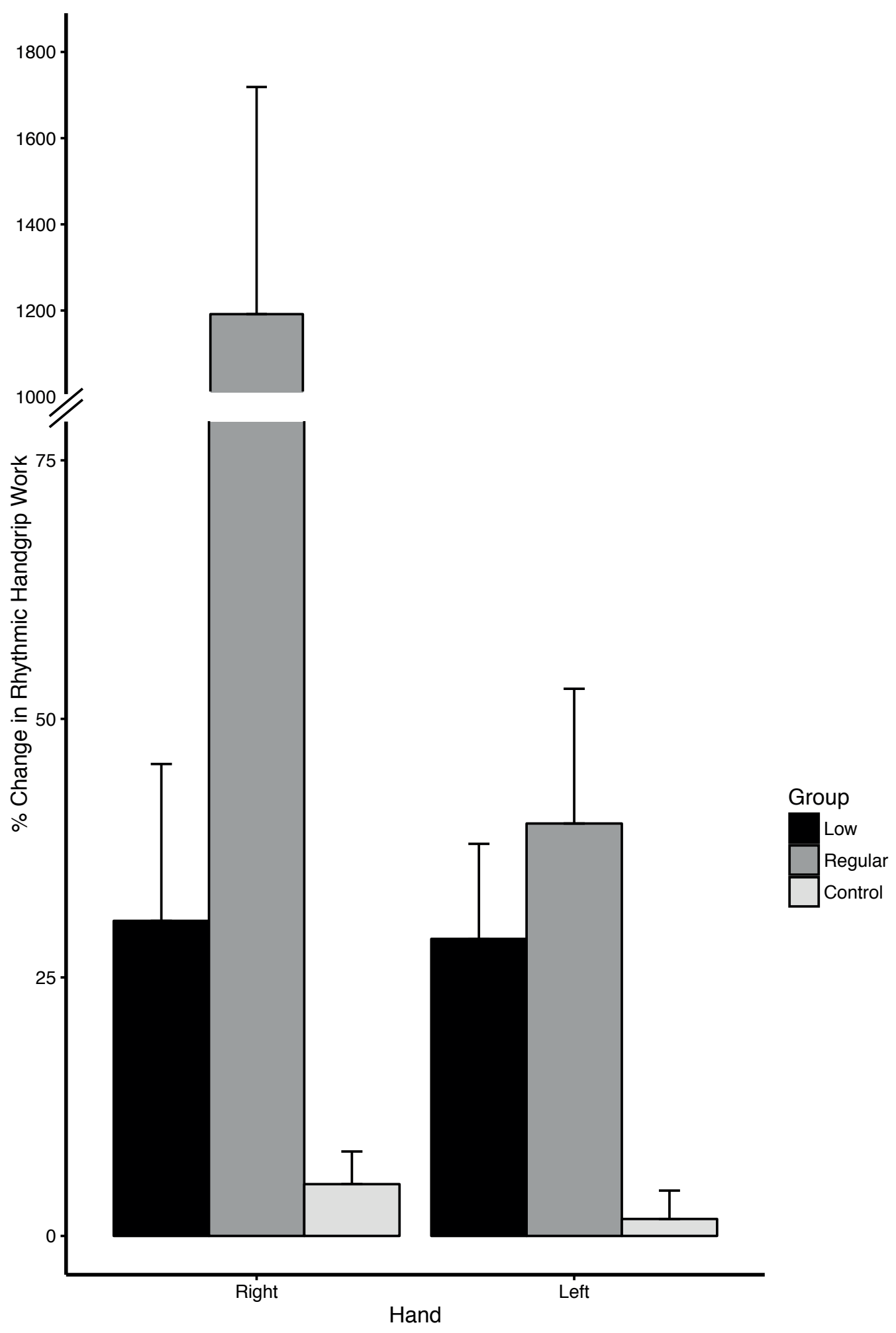

Two groups, each comprising 8 participants, performed submaximal rhythmic handgrip training for a period of six weeks. The low-level training group performed daily training with minimal load $(0.005 \%$ of maximal voluntary isometric contraction for the handgrip force (MVIC)). The regular training group trained with a load of $30 \%$ MVIC. An additional control group $(n=8)$ did not receive any training. Changes in maximal rhythmic handgrip work (RHW) from before the training period to after the training period (expressed as a percentage of initial values), are shown for the training limb (right) and non-training (left) limb. The error bars correspond to standard error of the means. This original plot by the author was generated using data extracted from Shields et al. (1999). These data illustrate that profound increases in grip strength may be brought about by interventions that do not alter the state of the muscle fibres. 


\section{Figure 6}

A

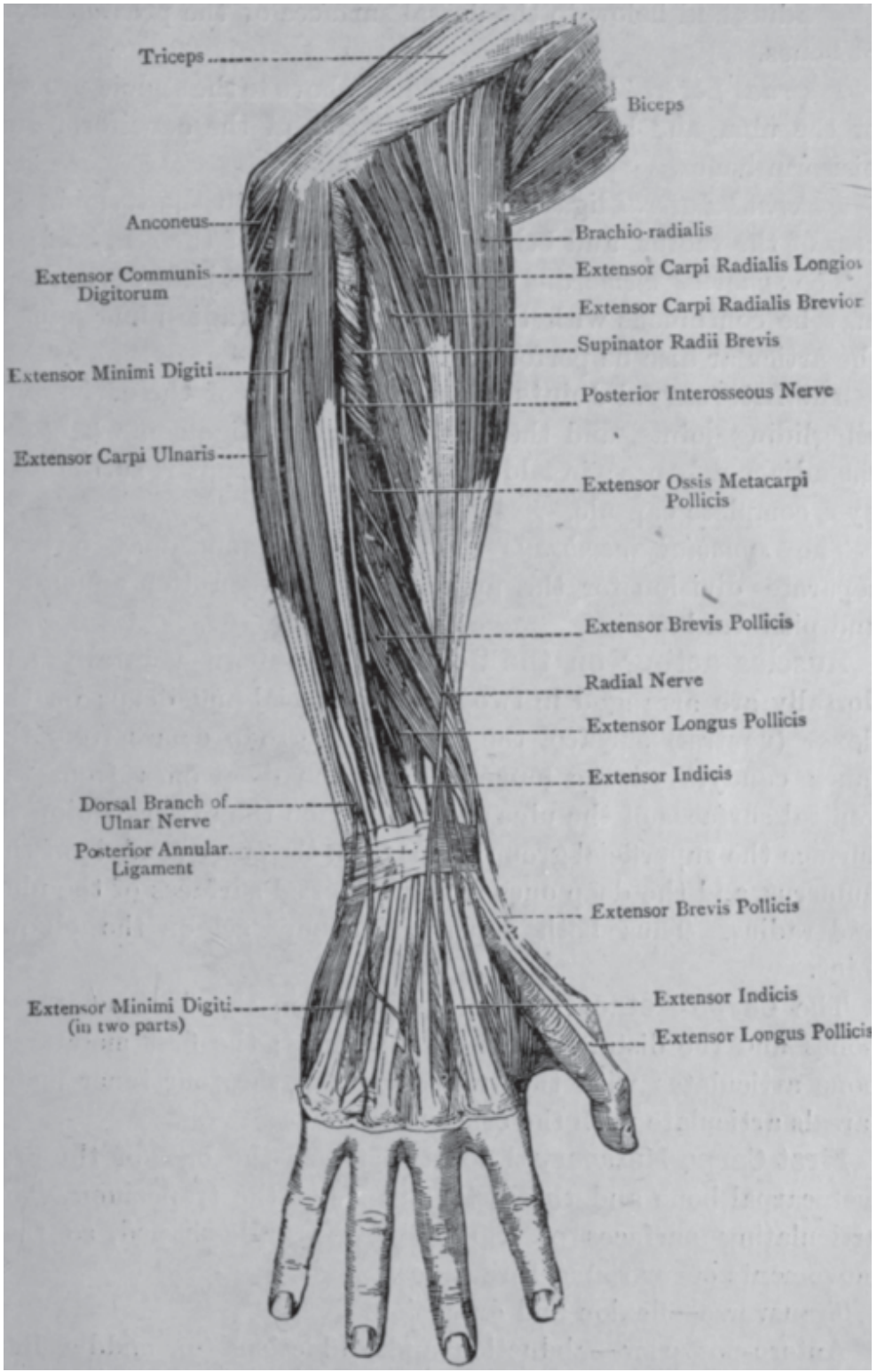

$\mathrm{B}$

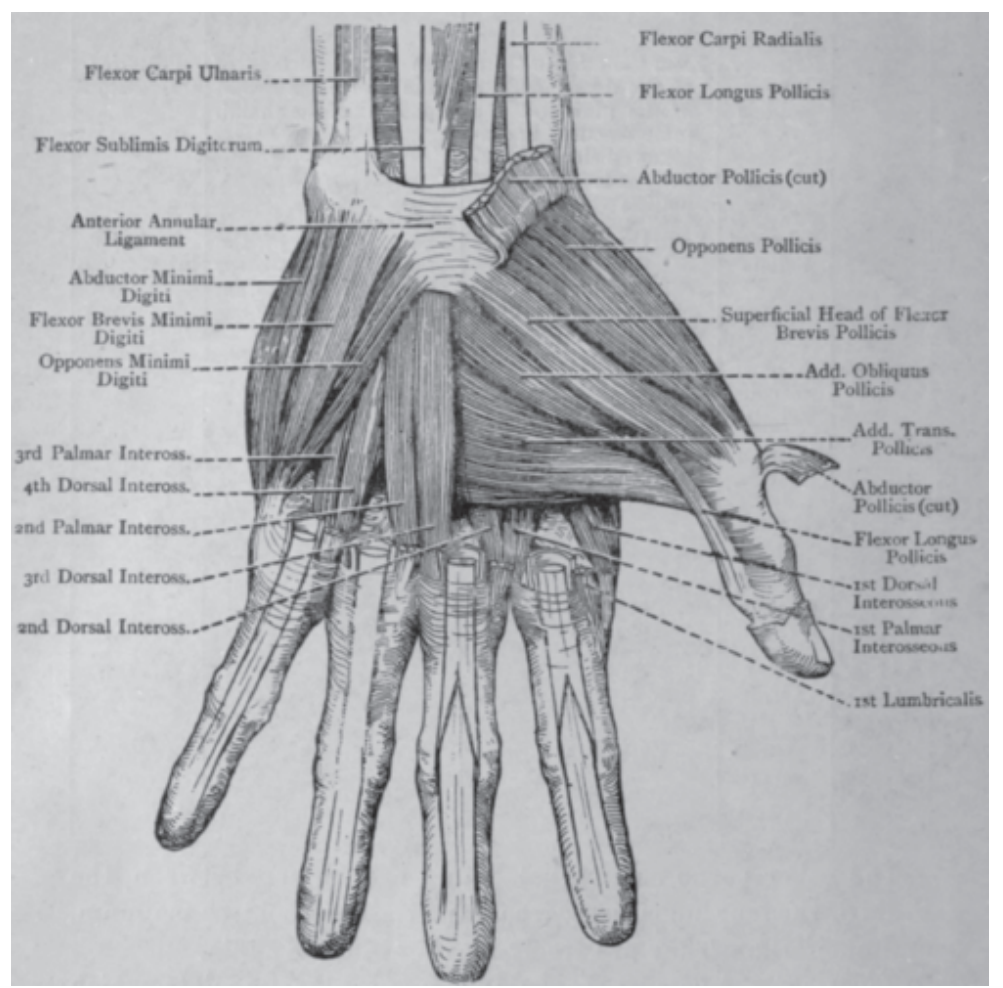

A graphical illustration of the muscles and tendons that give effect to prehensile movements of the human hand. These are shown in dorsal aspect (A) and palmar aspect (B). Reproduced from Handbook Of Anatomy For Students Of Massage by Margaret E. Bjorkegren. Copyright 1917, Bailliere, Tindall \& Cox. It can be seen that articulations of the hand form functional groups arranged in kinetic chains. These are subject to control via synergies constituted by the organised recruitment by the CNS of muscles extrinsic to the hand and muscles intrinsic to the hand, that when coordinated permit the differentiated action of the phalanxes. 
Figure 8

A

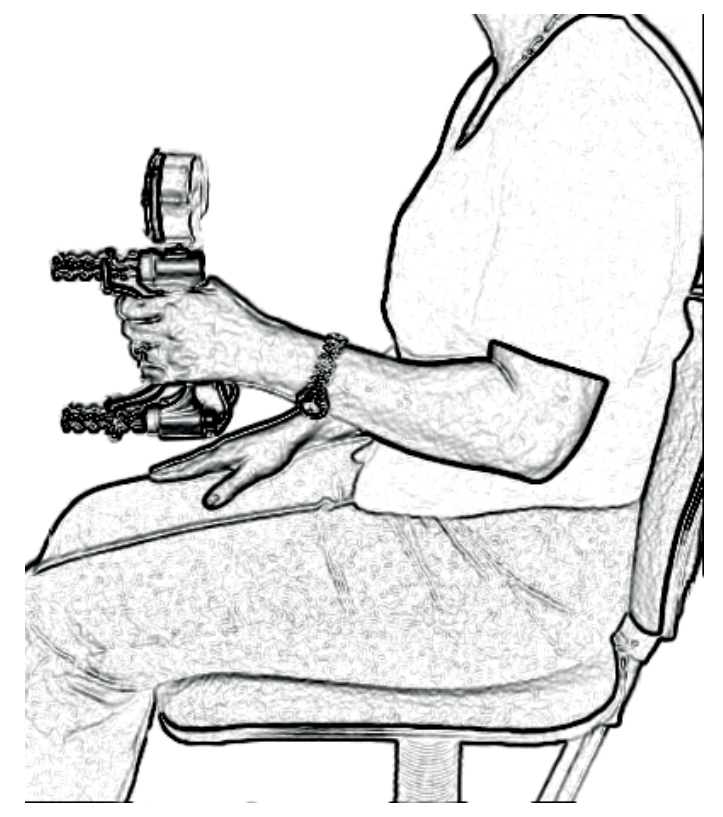

B

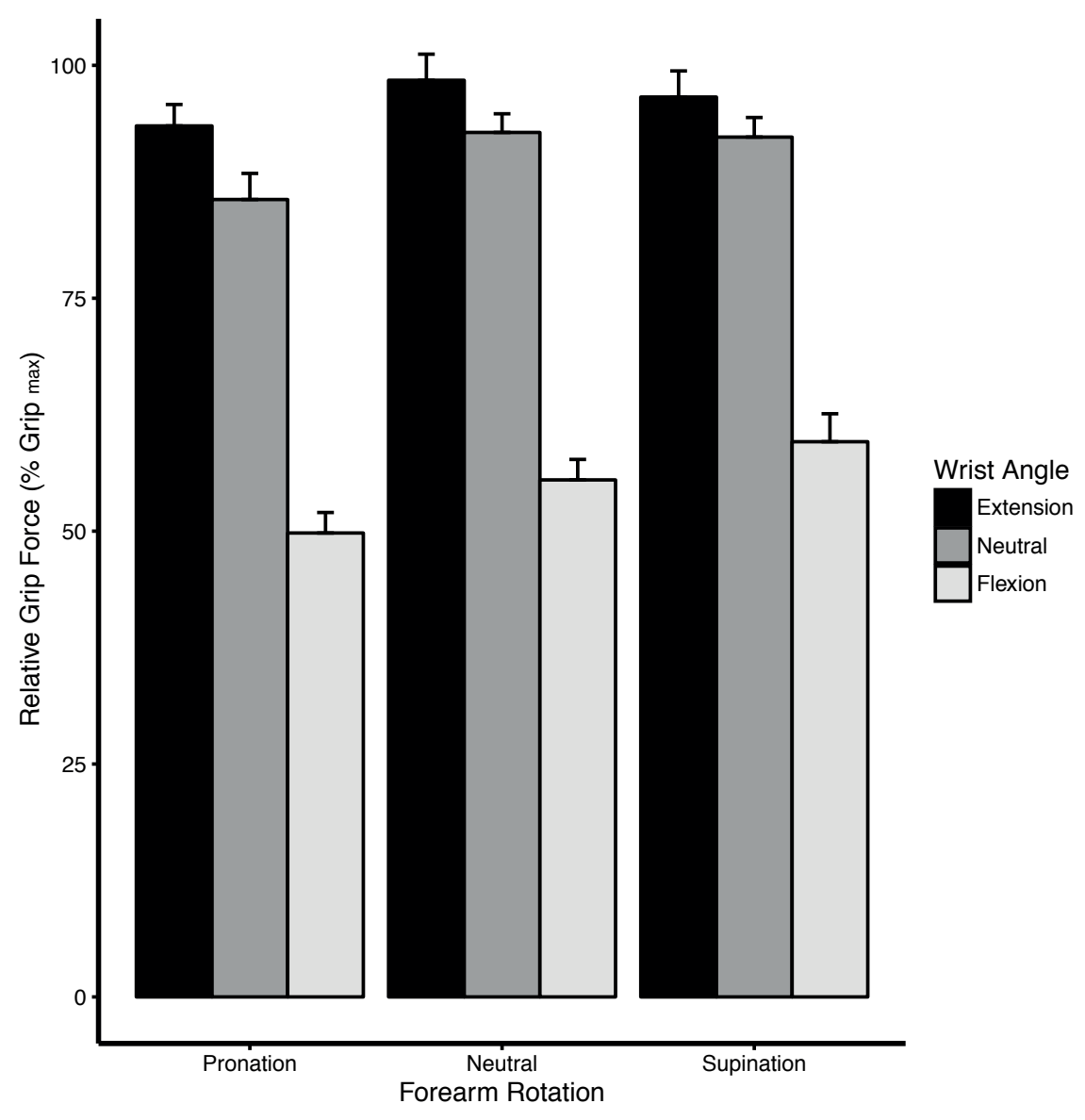

A: Standard configuration for the evaluation of maximum grip strength using a hand held dynamometer. B: Variations in maximum grip force as a function of forearm position and wrist angle obtained for 5 males and 5 females (mean age 23 years). The values (\% Grip max) are expressed as a percentage of the maximum values obtained in calibration trials conducted with the forearm and wrist in neutral positions. The error bars correspond to standard error of the means. This original plot by the author was generated using data extracted from Mogk and Keir (2003). The variation in grip force as a function of wrist joint angle (in flexion-extension) serves to reveal the impact of the biomechanical context in which the action is performed. 


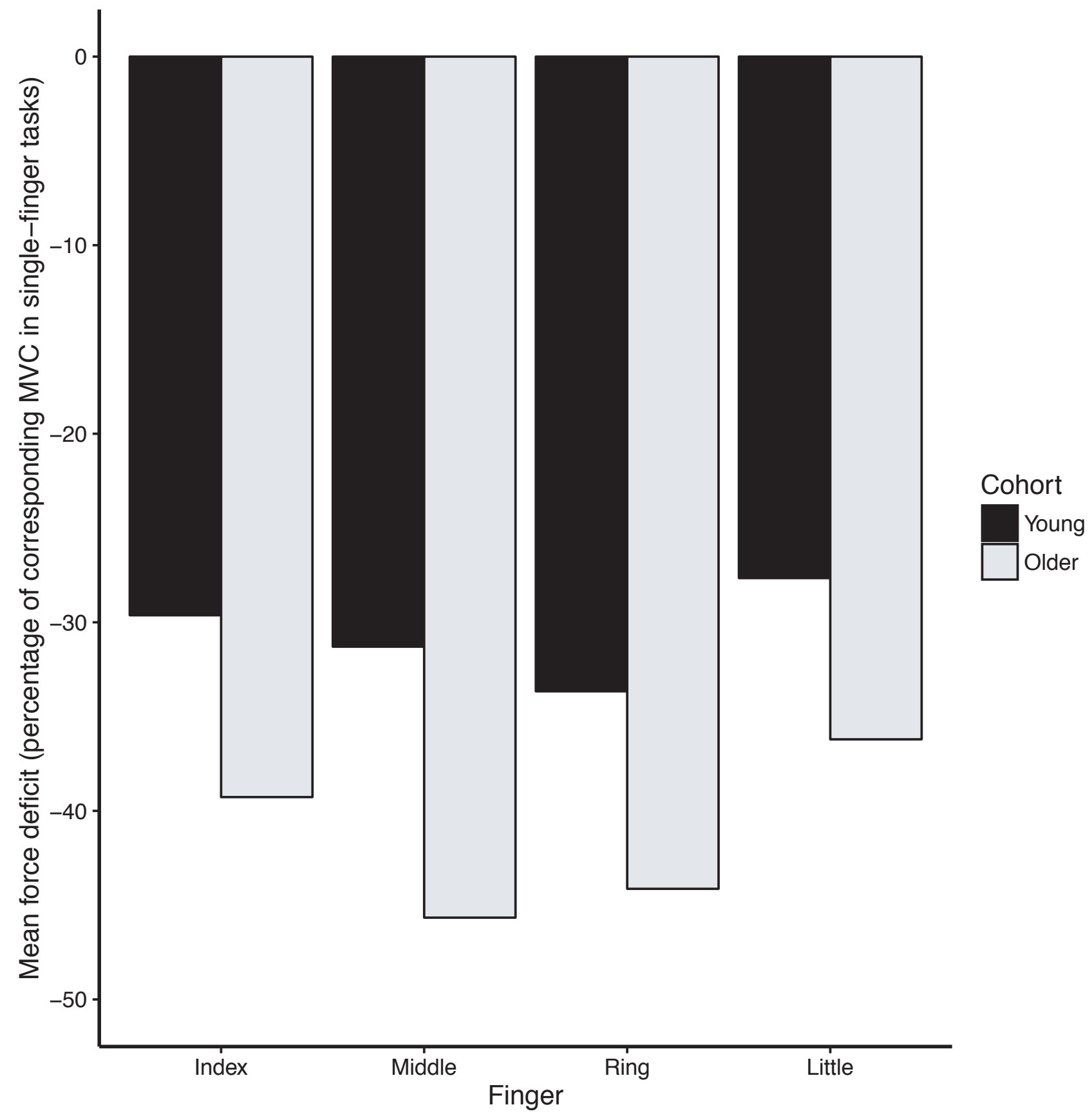

The multi-finger force deficit exhibited by a group of 6 male $(29.3 \pm 3.6 \mathrm{yr})$ and 6 female $(29.8 \pm 4.6 \mathrm{yr})$ young, and 6 male $(87.2 \pm 4.5 \mathrm{yr})$ and 6 female $(76.3 \pm 4.0)$ older participants. The deficit was calculated as the difference between the maximum force that could be generated by a finger in its single-finger test and that generated in the four-finger test, expressed as a percentage of the maximum value (MVC) in the single-finger test. The negative values indicate that the force that can be generated by each digit in the four-finger test is substantially lower than that produced when it is engaged in isolation. This original plot by the author was generated using data extracted from Shinohara et al. (2003a). It demonstrates that the diminution of the force applied by the individual digits during four-finger tasks is larger in older persons than in the young. 


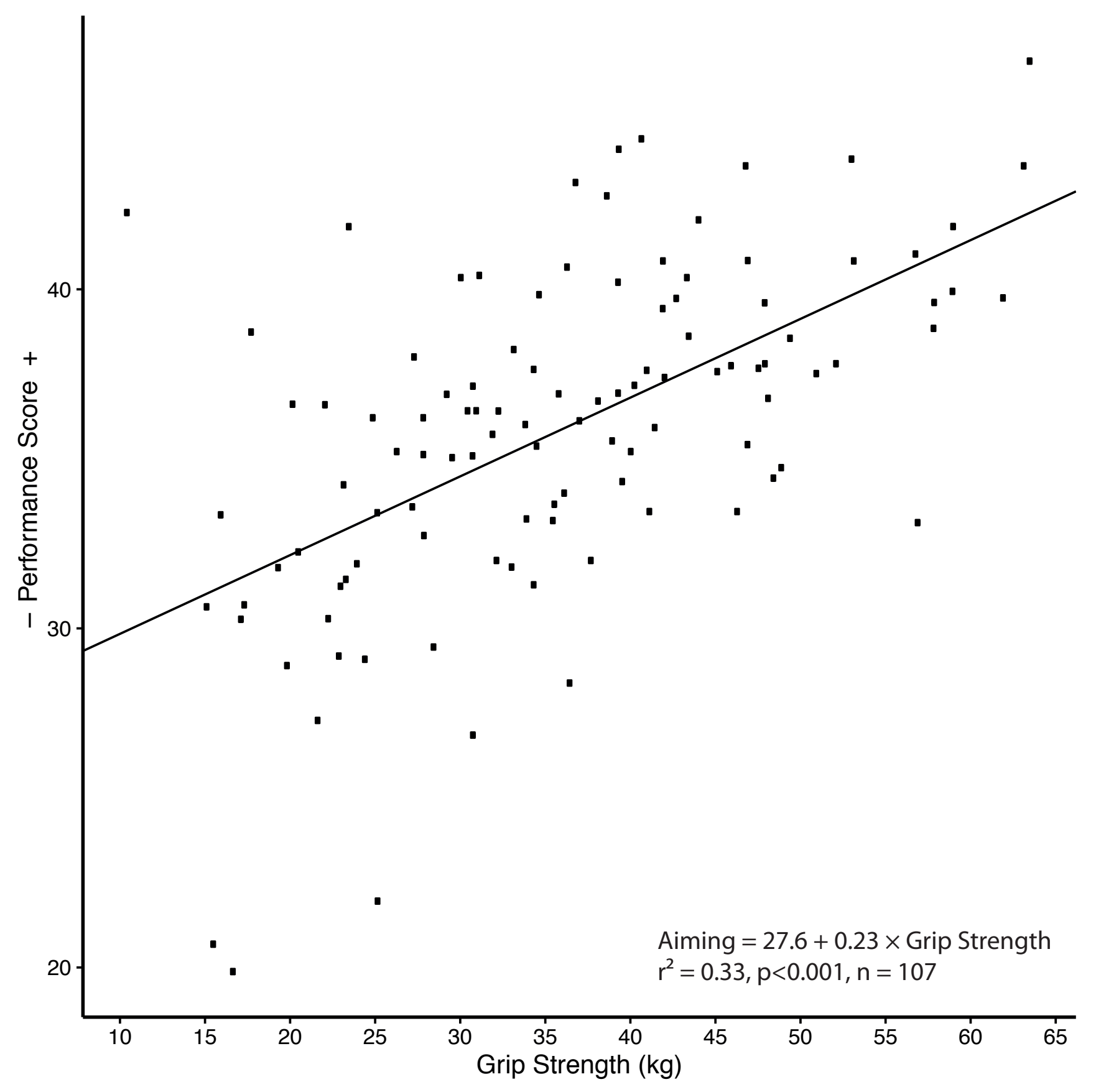

The relationship between grip strength and the aiming component of the 'Vienna Test System: Motor Performance Series Workboard', assessed in 107 participants, aged 18-93 years (60 female). This test requires that the participant touch with a pen stylus in sequence, 20 brass disks ( $5 \mathrm{~mm}$ diameter) arranged in a row. The distance between each disk is $4 \mathrm{~mm}$. The outcome measure is derived using the Fleishman-factor of weighted variables (i.e. as a composite of the number of touch errors and the total duration). The line of best fit obtained using simple linear regression, and the corresponding parameters of the fit are shown. This original plot by the author was generated using data extracted from Martin et al. (2015). These data illustrate that inter-individual variations in maximum grip strength are closely associated with variations in manual dexterity. As age increases, the strength of the association becomes greater. 


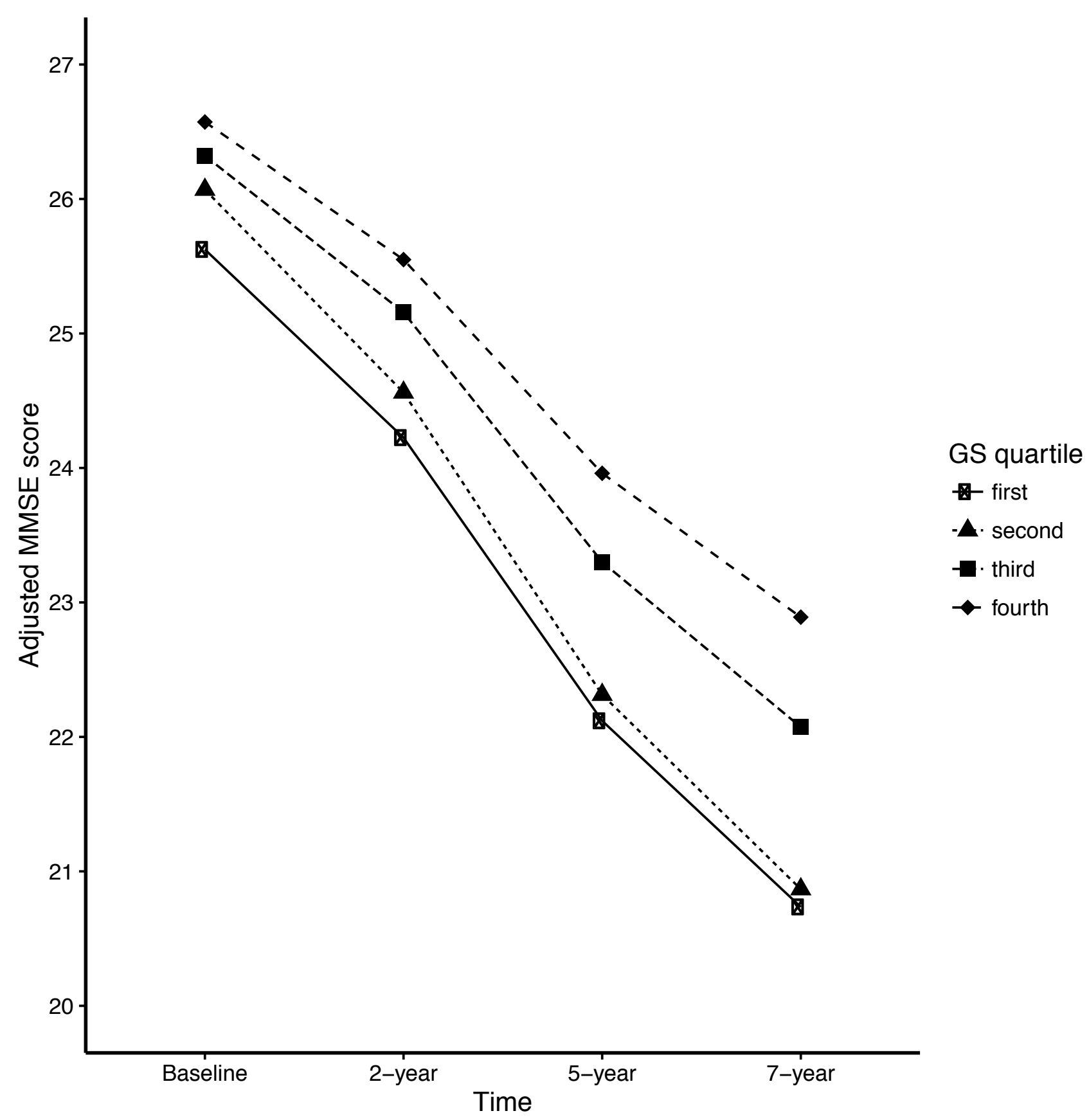

Means of the distributions of Mini-Mental State Examination (MMSE) scores obtained over a 7-year period, partitioned by handgrip strength quartiles at baseline in 2160 participants (1242 female; 918 male, with MMSE > 21 at baseline, and complete data on all covariates). The MMSE values are adjusted for age, gender, education, marital status, medical conditions, depression, near and distant vision impairment, and body mass index. This original plot by the author was generated using data extracted from Alfaro-Acha et al. (2006). It shows that maximum grip strength measured at the beginning of the assessment period predicts subsequent decreases in a multiple domain test of cognitive function. The extent of the decline is greatest for the group with the lowest grip strength on initial observation, and smallest for the group with the highest grip strength on initial observation. 


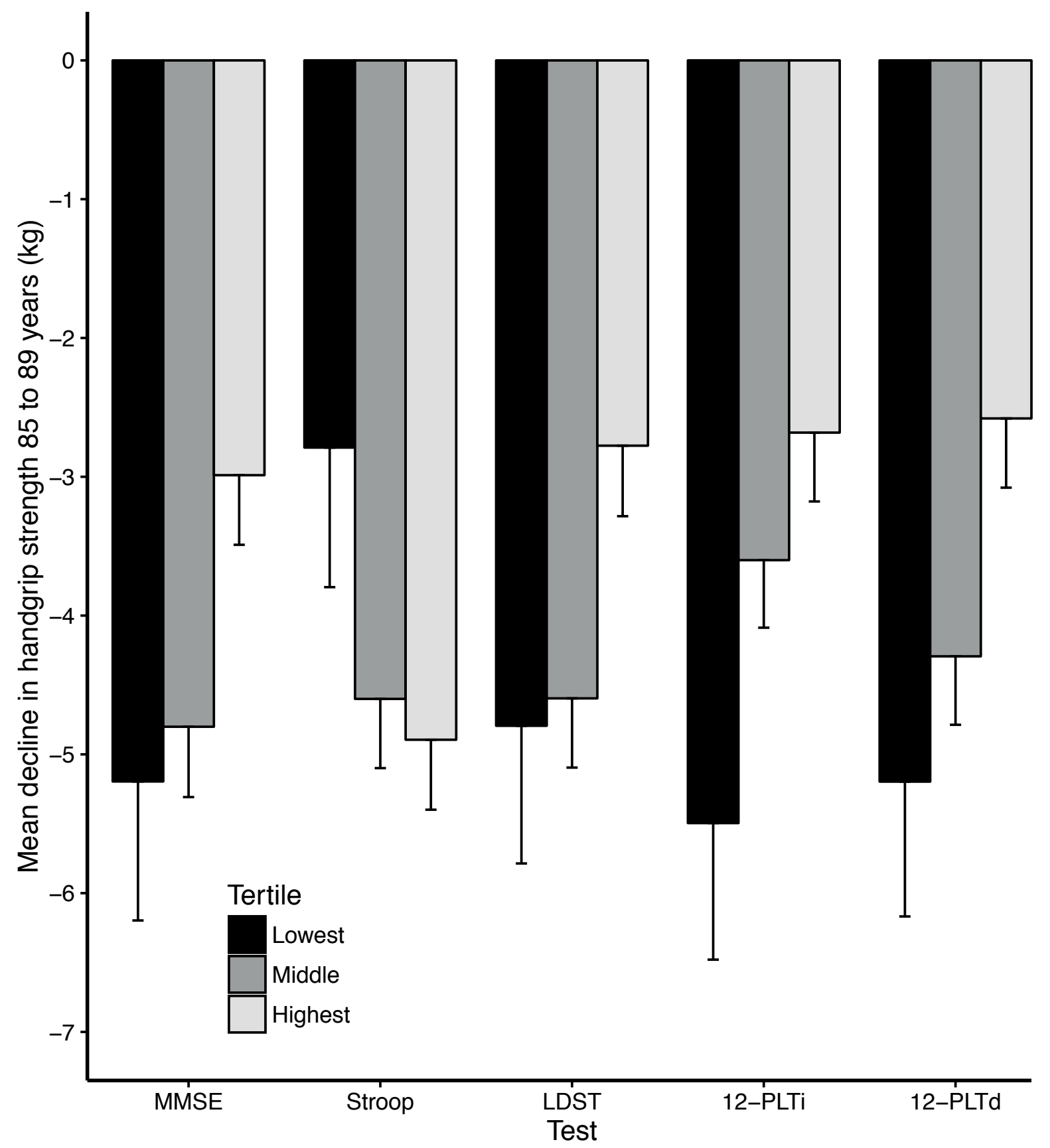

Changes in grip strength from age 85 to 89 years stratified by baseline cognitive performance tertile, for 307 (217 female; 90 male) participants drawn from the Leiden 85-plus Study. Cognitive performance was assessed on the basis of Mini-Mental State Examination (MMSE) scores; the abbreviated Stroop test; Letter Digit Substitution Task (LDST); and 12-Picture Learning Test (12-PLT immediate (i) and delayed recall (d)). Note that lower Stroop scores indicate better performance. The error bars correspond to standard error of the means. This original plot by the author was generated using data extracted from Taekema et al. (2012). It reveals that cognitive performance measured in multiple domains at the beginning of the assessment period, predicts subsequent decreases in grip strength. The extent of the decline in grip strength is greatest for the group with the lowest level of cognitive performance on initial observation, and smallest for the group with the highest level of cognitive performance on initial observation. 


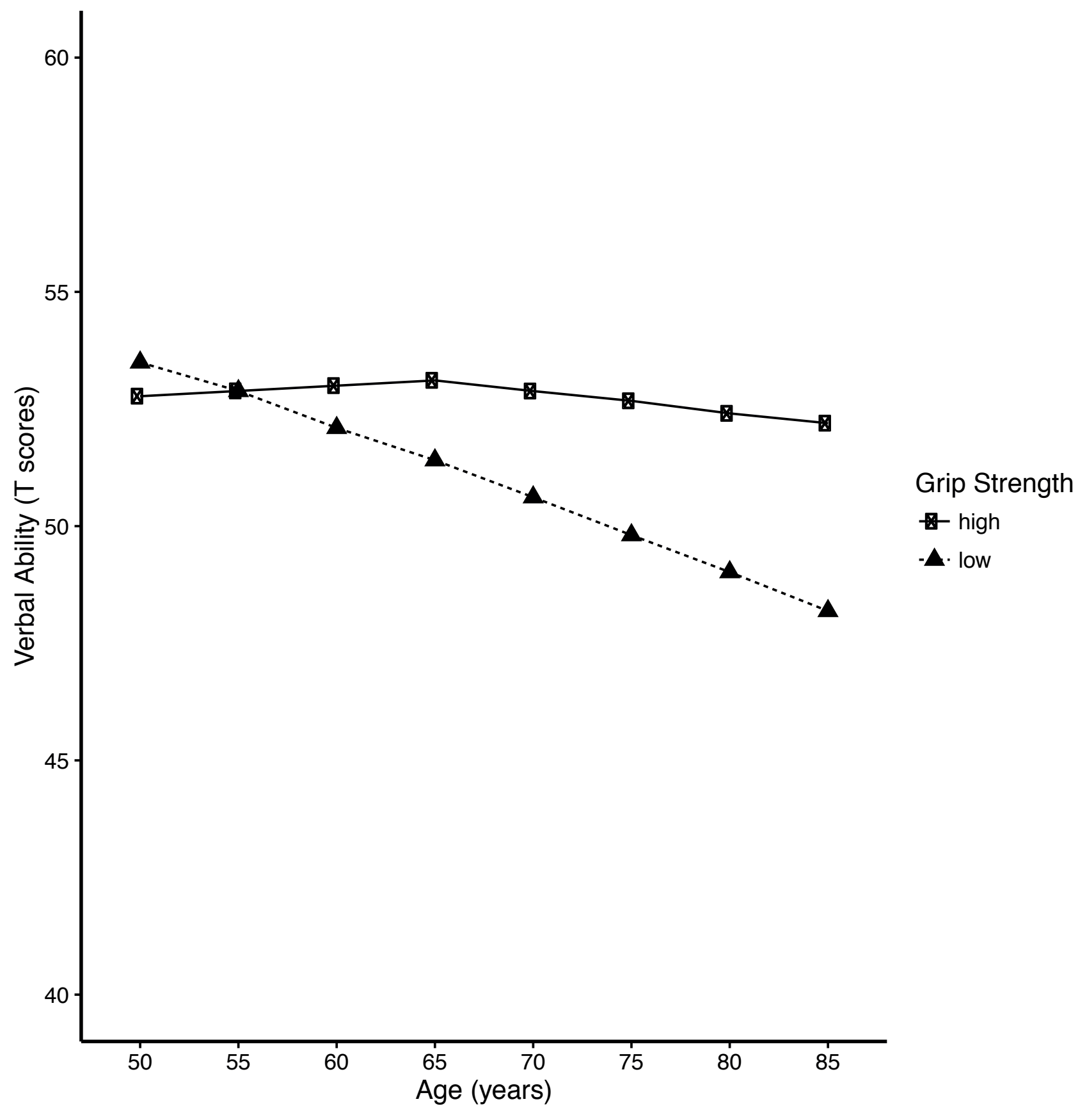

The relationship between initial grip strength classification (high +1 SD; low -1 SD) and subsequent changes in verbal ability (as assessed using the Information Subtest of the Wechsler Adult Intelligence Scale-Revised [WAIS-R]) in a group of 708 participants drawn from the population-based longitudinal Swedish

Adoption/Twin Study of Aging (397 female; 311 male, age range: 40-86 years at baseline). The measures of verbal ability obtained over the observation period were standardized by transformation to T-scores, using the factor means and variances derived for the first wave of testing. This original plot by the author was generated using data extracted from Sternäng et al. (2015). It illustrates that maximum grip strength measured at the beginning of the assessment period predicts subsequent decreases in verbal ability. The extent of the decline is greatest for the group with the lowest grip strength on initial observation, and smallest for the group with the highest grip strength on initial observation. 


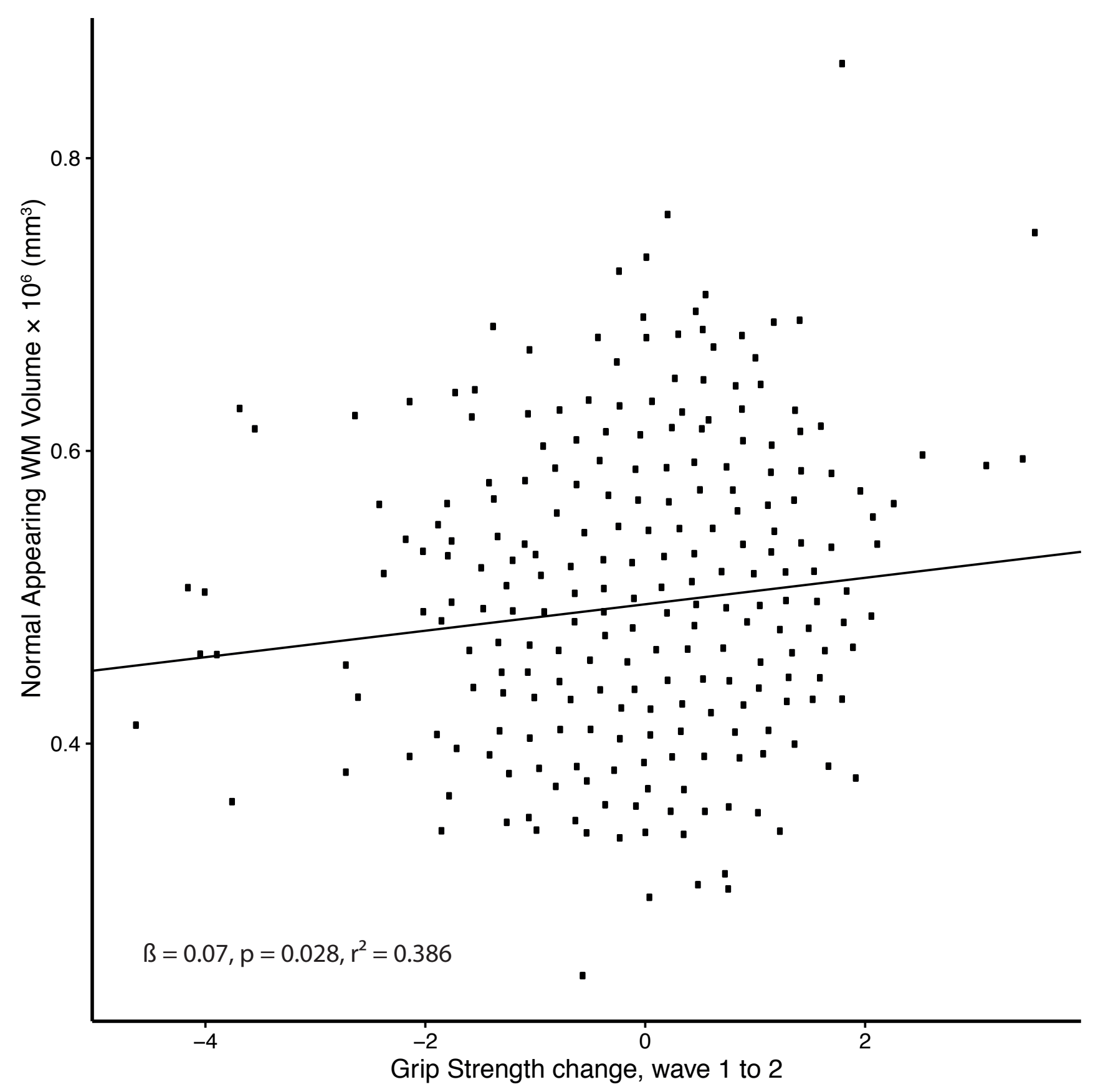

Relationship between the normal appearing white matter volume (estimated via MR imaging) assessed at age 73 years, and changes in grip strength from age 70 to 73, shown for a subgroup of participants drawn from the Lothian Birth Cohort 1936. The results $(n=694)$ of the corresponding regression analyses ( $R 2$ and an estimate of effect size - standardised $\beta$ ) are shown for a model that that included as covariates: age-11 IQ; years of education; social class; self-reported medical history of: cardiovascular disease, diabetes, hypertension, and stroke; and smoking status. This original plot by the author was generated using data extracted from Aribisala et al. (2013). It reveals that the volume of normal-appearing white matter (i.e. areas of white matter not affected by white matter lesions) at the beginning of the observation period, predicts subsequent decreases in grip strength. The extent of the decline in grip strength is greatest for the group with the lowest volumes on initial observation, and smallest for the group with the highest volumes on initial observation. 